\title{
Theoretical formalism of radiative jet energy loss in a finite size dynamical QCD medium
}

\author{
Magdalena Djordjevic* \\ Department of Chemistry and Physics, Arkansas State University, State University, Arkansas 72467, USA \\ and Physics Department, The Ohio State University, Columbus, Ohio 43210, USA
}

(Received 15 September 2009; published 21 December 2009)

\begin{abstract}
The computation of radiative energy loss in a finite-size QCD medium with dynamical constituents is a key ingredient for obtaining reliable predictions for jet quenching in ultrarelativistic heavy-ion collisions. We here present a theoretical formalism for the calculation of the first order in opacity radiative energy loss of a quark jet traveling through a finite-size dynamical QCD medium. We show that, while each individual contribution to the energy loss is infrared divergent, the divergence is naturally regulated once all diagrams are taken into account. Finite-size effects are shown to induce a nonlinear path-length dependence of the energy loss, recovering both the incoherent Gunion-Bertsch limit, as well as destructive Landau-Pomeanchuk-Migdal limit. Finally, our results suggest a remarkably simple general mapping between energy-loss expressions for static and dynamical QCD media.
\end{abstract}

DOI: 10.1103/PhysRevC.80.064909

PACS number(s): 25.75.Nq, 12.38.Mh, 12.38.Qk

\section{INTRODUCTION}

Suppression pattern of high transverse-momentum hadrons is a powerful tool to map out the density of a QCD plasma created in ultrarelativistic heavy-ion collisions [1-3]. Because suppression (called jet quenching) results from the energy loss of high-energy partons moving through the plasma [4-7], reliable theoretical predictions for suppression require reliable energy-loss calculations.

The medium-induced radiative energy loss is, in most studies, computed by assuming that the QCD medium consists of randomly distributed static scattering centers ("static QCD medium"). However, in reality, constituents of the medium are dynamical, and we recently showed that inclusion of dynamical QCD medium effects are important in the radiative energy-loss calculations [8,9]. Furthermore, calculation of the energy loss has to be performed in a finite-size QCD medium, because the size of the QCD medium created in both the Relativistic Heavy Ion Collider (RHIC) and the Large Hadron Collider (LHC) is finite. While methods for energy-loss calculation have been developed for infinite optically thick dynamical QCD medium, no such approach exists for finite optically thin medium. However, it is of crucial importance to develop the energy-loss formalism for the case of finite-size optically thin medium to make reliable predictions applicable for the range of parameters relevant for RHIC and LHC experiments.

This article develops a theoretical formalism for the calculation of the radiative energy loss in a finite-size dynamical QCD medium, while main numerical results of the model are presented elsewhere [9]. Our work is based a novel approach, where two hard thermal loops are implemented within a finite-size QCD model initially introduced by Zakharov [10]. The computations are presented in the appendices and include analytical calculations of 24 Feynman diagrams, each of which is individually infrared divergent. However, this divergence

*mdjordjevic@astate.edu is naturally regulated when all the diagrams are taken into account. Furthermore, we obtain an explicit analytical expression for the energy loss in dynamical medium, which can be directly compared with the equivalent expression obtained in static QCD medium. Finally, we discuss the obtained analytical result in the context of several qualitative features: (i) recovery of the static approximation for asymptotically high values of energy, (ii) transition of the thickness dependence from Gunion-Bertsch (GB) [11] to Landau-Pomeanchuk-Migdal (LPM) [12] limits, and (iii) possible extension of the results to higher orders in opacity.

\section{RADIATIVE ENERGY LOSS IN A DYNAMICAL QCD MEDIUM}

In this section we outline the computation of the mediuminduced radiative energy loss for a heavy quark to first order in opacity. We consider finite QCD medium of size $L$ and assume that the heavy quark is produced inside the medium at time $x_{0}=0$ at the left edge of the medium, traveling right.

Medium-induced radiative energy loss is caused by the radiation of one or more gluons induced by collisional interactions between the quark of interest and partons in the medium. The energy-loss rate can be expanded in the number of scattering events suffered by the heavy quark, which is equivalent to an expansion in powers of the opacity. For a finite medium, the opacity is given by the product of the density of the medium with the transport cross section, integrated along the path of the heavy quark. The lowest- (first-) order contribution corresponds to one collisional interaction with the medium, accompanied by emission of a single gluon.

We compute the medium-induced radiative energy loss for a quark jet to the first (lowest) order in number of scattering centers. The finite-size medium is introduced similarly as in Ref. [13], i.e., by starting from the approach described in Ref. [10]. That is, we assume that the medium has a size $L$ and that the collisional interaction has to occur inside the medium. 


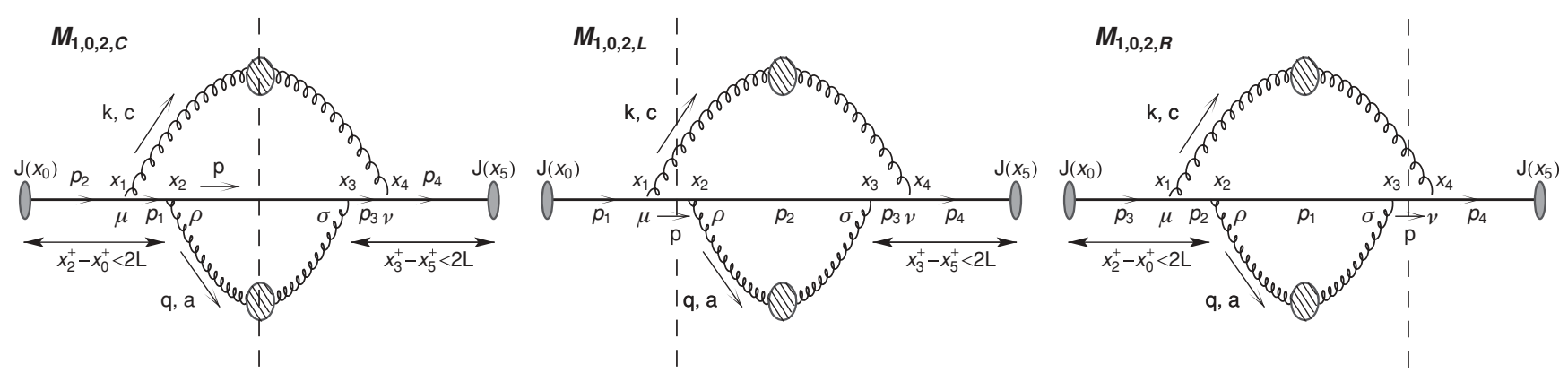

FIG. 1. Feynman diagrams $M_{1,0,2, C}, M_{1,0,2, L}$, and $M_{1,0,2, R}$ contribute to the radiative energy loss to the first order in the opacity. On each panel, the left (right) gray ellipse represents the source $J$, which at time $x_{0}\left(x_{5}\right)$ produces an on-shell jet with momentum $p_{2}\left(p_{5}\right)$. The large dashed circles ("blob") represent effective HTL gluon propagators [14]. A cut gluon propagator with momentum $k$ and color $c$ corresponds to the radiated gluon. A cut gluon propagator with momentum $q$ and color $a$ corresponds to a collisional interaction with a parton in the medium. Specific time points are represented by $x_{i}$. The diagrams are calculated in light cone coordinate system, and $x_{2}^{+}-x_{0}^{+}<2 L$ on the first and the third panels, as well as $x_{3}^{+}-x_{5}^{+}<2 L$ on the first and the second panels, represent the condition that the distance between collisional interaction and jet production has to be smaller than the size $L$ of the medium. (Left) Middle and right panels present three possible cuts (central, left, and right, respectively) of the same 2-HTL Feynman diagram, all of which contribute to the first order in opacity radiative energy loss.

As in Ref. [8], we describe the medium by a thermalized quark-gluon plasma at temperature $T$ and zero baryon density, with $n_{f}$ effective massless quark flavors in equilibrium with the gluons. The formalism for computing the energy loss in finite-size dynamical QCD medium is presented in Appendices $\mathrm{C}-\mathrm{N}$, and the diagrams are evaluated in finite temperature field theory [15,16], using HTL resumed propagators [16] for all gluons. To outline the calculations, in Fig. 1 we show three typical diagrams that have to be computed. The Feynman diagram in the left panel of Fig. 1 represents the source $J$, which at time $x_{0}$ produces an on-shell jet with momentum $p_{2}$ and subsequently radiates a gluon with momentum $k=\left(\omega, k_{z}, \boldsymbol{k}\right)$ and exchanges a virtual gluon of momentum $q=\left(q_{0}, q_{z}, \boldsymbol{q}\right)$ with a parton in the medium. The quark jet emerges with (measured) momentum $p=\left(E, p_{z}, \boldsymbol{p}\right)$. We assume, as in Ref. [17], that $J$ changes slowly with jet momentum, i.e., that $J(p+k+q) \approx J(p+k) \approx J(p)$. To incorporate the effect of the finite-size QCD medium, we assume that the distance between the jet production and collisional interaction has to be smaller than the size of the medium.

Because the produced jet can be off-shell, the Feynman diagrams shown in the center and right panels of Fig. 1 also contribute to the first order in opacity radiative energy loss. They are complex conjugates to each other and they present the terms that will interfere with the diagram shown in the left panel of Fig. 1 and consequently lead to the appearance of LPM effect (in the case of high-energy jets), after all relevant contributions are taken into account. In addition to the three diagrams shown in Fig. 1, there are 21 more diagrams that contribute to the first order in opacity radiative energy loss, and their calculation is presented in Appendices $\mathrm{C}-\mathrm{N}$. Note that the calculation presented in this article differs from Ref. [18] by the use of HTL gluon propagators to describe the interaction of the quark with the medium. The difference from Ref. [8] is that in this work we allow the jet to be on- or off-shell and the vertices that correspond to gluon exchange are restricted to be located inside the medium.
Because the exchanged gluon momentum is spacelike $[13,19,20])$, only the Landau damping contribution $\left(q_{0} \leqslant|\overrightarrow{\mathbf{q}}|\right)$ to the cut HTL effective gluon propagator $D(q)$ needs to be taken into account $[13,19,21]$. The radiated gluon has timelike momentum $k=(\omega, \overrightarrow{\mathbf{k}})$, so only the quasiparticle contribution at $\omega>|\overrightarrow{\mathbf{k}}|$ from the cut gluon propagator $D(k)$ contributes [14-16]. Because our focus is on heavy quarks with mass $M \gg g T$, we neglect the thermal shift of the heavy-quark mass.

The effective gluon propagator has both transverse and longitudinal contributions [22-28]. The 1-HTL gluon propagator has the form

$$
i D^{\mu \nu}(l)=\frac{P^{\mu \nu}(l)}{l^{2}-\Pi_{T}(l)}+\frac{Q^{\mu \nu}(l)}{l^{2}-\Pi_{L}(l)},
$$

where $l=\left(l_{0}, \overrightarrow{\mathbf{I}}\right)$ is the four-momentum of the gluon and $P_{\mu \nu}(l)$ and $Q_{\mu \nu}(l)$ are the transverse and longitudinal projectors, respectively. The transverse and longitudinal HTL gluon self-energies $\Pi_{T}$ and $\Pi_{L}$ are given by

$$
\begin{aligned}
& \Pi_{T}(l)=\mu^{2}\left[\frac{y^{2}}{2}+\frac{y\left(1-y^{2}\right)}{4} \ln \left(\frac{y+1}{y-1}\right)\right], \\
& \Pi_{L}(l)=\mu^{2}\left[1-y^{2}-\frac{y\left(1-y^{2}\right)}{2} \ln \left(\frac{y+1}{y-1}\right)\right],
\end{aligned}
$$

where $y \equiv l_{0} /|\overrightarrow{\mathbf{I}}|$ and $\mu=g T \sqrt{N_{c} / 3+N_{f} / 6}$ is the Debye screening mass.

While the results obtained in this article are gauge invariant [20], the calculation is for simplicity presented in Coulomb gauge. In this gauge the only nonzero terms in the transverse and longitudinal projectors are

$$
\begin{aligned}
P^{i j}(l) & =-\delta^{i j}+\frac{l^{i} l^{j}}{\overrightarrow{\mathbf{l}}^{2}}, \\
Q^{00}(l) & =-\frac{l^{2}}{\overrightarrow{\mathbf{l}}^{2}}=1-\frac{l_{0}^{2}}{\overrightarrow{\mathbf{l}}^{2}}=1-y^{2} .
\end{aligned}
$$


As in Refs. [17,18,29-34], we use the same kinematic approximations, i.e., we assume validity of the soft-gluon $(\omega \ll E)$ and soft-rescattering $\left(\omega \gg|\boldsymbol{k}| \sim|\boldsymbol{q}| \sim q_{0}, q_{z}\right)$ approximations (see Appendix A for details). In Appendices $\mathrm{C}-\mathrm{N}$ we compute all the diagrams that contribute to the first order in opacity radiative energy loss. Once the diagrams are calculated, the interaction rate is given by:

$$
\Gamma(E)=\frac{1}{N_{J}} M_{\mathrm{tot}}=\frac{1}{N_{J}}\left(M_{1,0}+M_{1,1}+M_{1,2}\right),
$$

here $M_{1,0}, M_{1,1}$, and $M_{1,2}$ present the sum of all contributions in which zero, one, or two (respectively) ends of the exchanged gluon $q$ are attached to the radiated gluon $k$. Furthermore, $N_{J}$ is an integrated invariant distribution of jets, created by the effective jet source current and given by [17] (note that $D_{R}=3$ accounts for the jet colors)

$$
N_{J}=D_{R} \int \frac{d^{3} p}{(2 \pi)^{3} 2 E}|J(p)|^{2} .
$$

Equations (C16), (D13), (D29), (E7), (F12), and (G7) give final results for the Feynman diagrams contributing to $M_{1,0}$. After adding these expressions $M_{1,0}$ becomes

$$
\begin{aligned}
M_{1,0}= & 4 L \operatorname{Lg}^{4}\left[t_{a}, t_{c}\right]\left[t_{c}, t_{a}\right] \int \frac{d^{3} p}{(2 \pi)^{3} 2 E}|J(p)|^{2} \\
& \times \int \frac{d^{3} k}{(2 \pi)^{3} 2 \omega} \frac{d^{2} q}{(2 \pi)^{2}} \frac{\mu^{2}}{\boldsymbol{q}^{2}\left(\boldsymbol{q}^{2}+\mu^{2}\right)} \times \frac{\boldsymbol{k}^{2}}{\left(\boldsymbol{k}^{2}+\chi\right)^{2}} \\
& \times\left[1-\frac{\sin (\xi L)}{\xi L}\right], \\
= & D_{R} \int \frac{d^{3} p}{(2 \pi)^{3} 2 E}|J(p)|^{2} \frac{C_{R} \alpha_{s}}{\pi} \frac{L}{\lambda_{\mathrm{dyn}}} \int \frac{d x}{x} \frac{d^{2} k}{\pi} \frac{d^{2} q}{\pi} \\
& \times \frac{\mu^{2}}{\boldsymbol{q}^{2}\left(\boldsymbol{q}^{2}+\mu^{2}\right)} \times \frac{\boldsymbol{k}^{2}}{\left(\boldsymbol{k}^{2}+\chi\right)^{2}}\left[1-\frac{\sin (\xi L)}{\xi L}\right],
\end{aligned}
$$

where $\chi \equiv M^{2} x^{2}+m_{g}^{2}$ and $\xi \equiv \frac{k^{2}+\chi}{x E^{+}}$are defined by Eq. (A8) in Appendix A.

Here and below we used $\left[t_{a}, t_{c}\right]\left[t_{c}, t_{a}\right]=C_{2}(G) C_{R} D_{R}$ [with $C_{2}(G)=3, C_{R}=\frac{4}{3}$, and $\left.D_{R}=3\right]$ and defined a "dynamical mean free path" (see Ref. [8]) $\lambda_{\text {dyn }}$ through

$$
\lambda_{\text {dyn }}^{-1} \equiv C_{2}(G) \alpha_{s} T=3 \alpha_{s} T,
$$

where $\alpha_{s}=\frac{g^{2}}{4 \pi}$ is coupling constant, and we assumed constant coupling $g$.

Equations (H11), (I8), (J10), and (K10) give final results for the Feynman diagrams contributing to $M_{1,1}$. After adding these expressions $M_{1,1}$ becomes

$$
\begin{aligned}
M_{1,1}= & D_{R} \int \frac{d^{3} p}{(2 \pi)^{3} 2 E}|J(p)|^{2} \frac{C_{R} \alpha_{s}}{\pi} \frac{L}{\lambda_{\mathrm{dyn}}} \\
& \times \int \frac{d x}{x} \frac{d^{2} k}{\pi} \frac{d^{2} q}{\pi} \frac{\mu^{2}}{\boldsymbol{q}^{2}\left(\boldsymbol{q}^{2}+\mu^{2}\right)} \\
& \times \frac{-2 \boldsymbol{k} \cdot(\boldsymbol{k}+\boldsymbol{q})}{\left(\boldsymbol{k}^{2}+\chi\right)\left[(\boldsymbol{k}+\boldsymbol{q})^{2}+\chi\right]}\left[1-\frac{\sin (\zeta L)}{\zeta L}\right],
\end{aligned}
$$

where $\zeta \equiv \frac{(\boldsymbol{k}+\boldsymbol{q})^{2}+\chi}{x E^{+}}$is defined by Eq. (A8) in Appendix A.
Equations (L7) and (M6) give final results for the Feynman diagrams contributing to $M_{1,2}$. After adding these expressions $M_{1,2}$ becomes

$$
\begin{aligned}
M_{1,2}= & D_{R} \int \frac{d^{3} p}{(2 \pi)^{3} 2 E}|J(p)|^{2} \frac{C_{R} \alpha_{s}}{\pi} \frac{L}{\lambda_{\mathrm{dyn}}} \\
& \times \int \frac{d x}{x} \frac{d^{2} k}{\pi} \frac{d^{2} q}{\pi} \frac{\mu^{2}}{\boldsymbol{q}^{2}\left(\boldsymbol{q}^{2}+\mu^{2}\right)}\left\{\frac{2(\boldsymbol{k}+\boldsymbol{q})^{2}}{\left[(\boldsymbol{k}+\boldsymbol{q})^{2}+\chi\right]^{2}}\right. \\
& \left.\times\left[1-\frac{\sin (\zeta L)}{\zeta L}\right]-\frac{\boldsymbol{k}^{2}}{\left(\boldsymbol{k}^{2}+\chi\right)^{2}}\left[1-\frac{\sin (\xi L)}{\xi L}\right]\right\} .
\end{aligned}
$$

By using Eqs. (2.5)-(2.9) the interaction rate [Eq. (2.4)] reduces to

$$
\begin{aligned}
\Gamma(E)= & \frac{C_{R} \alpha_{s}}{\pi} \frac{L}{\lambda_{\mathrm{dyn}}} \int \frac{d x}{x} \frac{d^{2} k}{\pi} \frac{d^{2} q}{\pi} \frac{\mu^{2}}{\boldsymbol{q}^{2}\left(\boldsymbol{q}^{2}+\mu^{2}\right)} \frac{2(\boldsymbol{k}+\boldsymbol{q})}{(\boldsymbol{k}+\boldsymbol{q})^{2}+\chi} \\
& \times\left[\frac{\boldsymbol{k}+\boldsymbol{q}}{(\boldsymbol{k}+\boldsymbol{q})^{2}+\chi}-\frac{\boldsymbol{k}}{\boldsymbol{k}^{2}+\chi}\right]\left[1-\frac{\sin (\zeta L)}{\zeta L}\right] .
\end{aligned}
$$

The heavy-quark radiative energy loss per unit length is obtained from the above expression for the interaction rate by weighting it with the lost energy $\omega+q_{0}$. In the soft-rescattering approximation $\omega+q_{0} \approx \omega$, leading to:

$$
\frac{d E_{\mathrm{dyn}}}{d L}=\frac{1}{D_{R}} \int d \omega \omega \frac{d \Gamma(E)}{d \omega} \approx \frac{E}{D_{R}} \int d x x \frac{d \Gamma(E)}{d x} .
$$

This finally leads to

$$
\begin{aligned}
& \frac{\Delta E_{\mathrm{dyn}}}{E} \\
& =\frac{C_{R} \alpha_{s}}{\pi} \frac{L}{\lambda_{\mathrm{dyn}}} \int d x \frac{d^{2} k}{\pi} \frac{d^{2} q}{\pi} \frac{\mu^{2}}{\boldsymbol{q}^{2}\left(\boldsymbol{q}^{2}+\mu^{2}\right)} \times 2 \frac{\boldsymbol{k}+\boldsymbol{q}}{(\boldsymbol{k}+\boldsymbol{q})^{2}+\chi} \\
& \quad \times\left[\frac{\boldsymbol{k}+\boldsymbol{q}}{(\boldsymbol{k}+\boldsymbol{q})^{2}+\chi}-\frac{\boldsymbol{k}}{\boldsymbol{k}^{2}+\chi}\right]\left\{1-\frac{\sin \left[\frac{(\boldsymbol{k}+\boldsymbol{q})^{2}+\chi}{x E^{+}} L\right]}{\frac{(\boldsymbol{k}+\boldsymbol{q})^{2}+\chi}{x E^{+}} L}\right\} .
\end{aligned}
$$

It is important to note that, similarly to Ref. [8], each individual diagram that contributes to the energy loss in a finite-size dynamical QCD medium diverges logarithmically in the limit of zero transverse momentum of the exchanged gluon, $\boldsymbol{q} \rightarrow 0$. The reason for this divergence is that in a dynamical QCD medium both transverse and longitudinal gluon exchange contribute to the radiative energy loss [35]. While Debye screening makes the longitudinal gluon exchange infrared finite, transverse gluon exchange causes a well-known logarithmic singularity [16] due to the absence of a magnetic screening [36]. Remarkably, we see from Eq. (2.12) that, when the contributions from all diagrams are taken into account, the infrared divergences cancel, naturally regulating the energy-loss rate. 
The analytical expression for the energy loss in dynamical medium that we obtained can now be directly compared with the equivalent expression for static QCD medium. We below make this comparison to study the importance of the dynamical QCD medium effects. To do that, we here rewrite the expression for the DGLV first order in opacity radiative energy loss in static QCD medium, which is obtained in Ref. [18]:

$$
\begin{aligned}
& \frac{\Delta E_{\text {stat }}}{E} \\
& =\frac{C_{R} \alpha_{s}}{\pi} \frac{L}{\lambda_{\text {stat }}} \int d x \frac{d^{2} k}{\pi} \frac{d^{2} q}{\pi} \frac{\mu^{2}}{\left(\boldsymbol{q}^{2}+\mu^{2}\right)^{2}} \times 2 \frac{\boldsymbol{k}+\boldsymbol{q}}{(\boldsymbol{k}+\boldsymbol{q})^{2}+\chi} \\
& \quad \times\left[\frac{\boldsymbol{k}+\boldsymbol{q}}{(\boldsymbol{k}+\boldsymbol{q})^{2}+\chi}-\frac{\boldsymbol{k}}{\boldsymbol{k}^{2}+\chi}\right]\left\{1-\frac{\sin \left[\frac{(\boldsymbol{k}+\boldsymbol{q})^{2}+\chi}{x E^{+}} L\right]}{\frac{(\boldsymbol{k}+\boldsymbol{q})^{2}+\chi}{x E^{+}} L}\right\},
\end{aligned}
$$

with $[8,37]$

$$
\frac{1}{\lambda_{\text {stat }}}=\frac{1}{\lambda_{g}}+\frac{1}{\lambda_{q}}=6 \frac{1.202}{\pi^{2}} \frac{1+\frac{n_{f}}{4}}{1+\frac{n_{f}}{6}} 3 \alpha_{s} T=c\left(n_{f}\right) \frac{1}{\lambda_{\mathrm{dyn}}},
$$

where $c\left(n_{f}\right) \equiv 6 \frac{1.202}{\pi^{2}} \frac{1+n_{f} / 4}{1+n_{f} / 6}$ is a slowly increasing function of $n_{f}$ that varies between $c(0) \approx 0.73$ and $c(\infty) \approx 1.09$. For a typical value $n_{f}=2.5$ (which we use in this article), $c(2.5) \approx$ 0.84 .

We see that, similarly to the case of infinite QCD medium [8], Eqs. (2.12) and (2.13) are remarkably similar, up to two important differences: The first is an $\mathcal{O}(15 \%)$ decrease in the effective mean free path

$$
\lambda_{\text {dyn }} \Longleftrightarrow \lambda_{\text {stat }}=\frac{\lambda_{\text {dyn }}}{c\left(n_{f}\right)},
$$

which increases the energy-loss rate in the dynamical medium by $\mathcal{O}(20 \%)$. The second difference is a change in the effective cross section, which in the energy-loss rate is reflected by the replacement

$$
\left[\frac{\mu^{2}}{\boldsymbol{q}^{2}\left(\boldsymbol{q}^{2}+\mu^{2}\right)}\right]_{\mathrm{dyn}} \Longleftrightarrow\left[\frac{\mu^{2}}{\left(\boldsymbol{q}^{2}+\mu^{2}\right)^{2}}\right]_{\text {stat }} .
$$

As discussed in Ref. [9], these differences lead to a significant increase of the heavy-quark energy-loss rate in dynamical compared to static QCD medium.

By using the above results, we can now discuss the following two issues: (i) comparison between dynamical and static energy-loss results and (ii) comparison between energyloss results in Bethe-Heitler limit and finite-size QCD medium. Regarding the first comparison, one should note a remarkably simple mapping between dynamical and static QCD medium. That is, the expression for energy loss in dynamical QCD medium can be obtained from the expression for the energy loss in static QCD medium by simply replacing the effective mean free path and the effective cross section by the appropriate expressions given above. The simplicity of this substitution rule is surprising, given the complexity of the calculations and their different structure for static [18] and dynamical media. In particular, one should note the infrared divergences in the dynamical case that cancel only after summing all the diagrams from Appendices $\mathrm{C}-\mathrm{N}$, but do not arise at all in the static case. Regarding the second comparison, the expressions for both dynamical and static energy loss in finite-size QCD medium are significantly different from the corresponding expressions in Bethe-Heitler limit [8]. However, despite this difference, the same simple substitution rule is found to apply, suggesting a possibly general mapping between static and dynamic QCD media.

We also note that the study presented here considers a finite, optically thin dynamical QCD medium [quark-gluon plasma (QGP)], extending the DGLV approach $[17,18]$ to include parton recoil. In this sense it is complementary to the work by Arnold, Moore, and Yaffe [34] who study energy loss in an infinite, optically thick QGP. We note that the AMY approach [34] yields the same form (2.16) for the effective cross section in a dynamical QCD medium as found here (see also Ref. [38]), further supporting our conjecture above.

\section{INCOHERENT LIMIT}

First of the two relevant limits of the Eq. (2.12), which we consider in this article is the incoherent, short formation time limit. This limit is relevant for heavy quarks at lower jet energy regions, and it can be extracted from the Eq. (2.12)

$$
\begin{aligned}
& \text { when } \frac{\sin \left[\frac{(k+q)^{2}+x}{x E^{+}} L\right]}{\frac{(k+q)^{2}+x}{x E^{+}} L} \rightarrow 0 \text { : } \\
& \frac{\Delta E_{\mathrm{dyn}}}{E}=\frac{C_{R} \alpha_{s}}{\pi} \frac{L}{\lambda_{\mathrm{dyn}}} \int d x \frac{d^{2} k}{\pi} \frac{d^{2} q}{\pi} \frac{\mu^{2}}{\boldsymbol{q}^{2}\left(\boldsymbol{q}^{2}+\mu^{2}\right)} \frac{2(\boldsymbol{k}+\boldsymbol{q})}{(\boldsymbol{k}+\boldsymbol{q})^{2}+\chi} \\
& \times\left[\frac{\boldsymbol{k}+\boldsymbol{q}}{(\boldsymbol{k}+\boldsymbol{q})^{2}+\chi}-\frac{\boldsymbol{k}}{\boldsymbol{k}^{2}+\chi}\right] \\
& =\frac{C_{R} \alpha_{s}}{\pi} \frac{L}{\lambda_{\mathrm{dyn}}} \int d x d \boldsymbol{k}^{2} d \boldsymbol{q}^{2} \frac{\mu^{2}}{\boldsymbol{q}^{2}\left(\boldsymbol{q}^{2}+\mu^{2}\right)} \frac{1}{\boldsymbol{k}^{2}+\chi} \\
& \times\left\{\frac{\boldsymbol{k}^{2}+3 \boldsymbol{q}^{2}+\chi}{\sqrt{\chi^{2}+2 \chi\left(\boldsymbol{k}^{2}+\boldsymbol{q}^{2}\right)+\left(\boldsymbol{k}^{2}-\boldsymbol{q}^{2}\right)^{2}}}-1\right. \\
& \left.-\frac{2 \boldsymbol{q}^{2}\left[\left(\boldsymbol{k}^{2}-\boldsymbol{q}^{2}\right)^{2}+\chi\left(5 \boldsymbol{k}^{2}+\boldsymbol{q}^{2}\right)\right]}{\left[\chi^{2}+2 \chi\left(\boldsymbol{k}^{2}+\boldsymbol{q}^{2}\right)+\left(\boldsymbol{k}^{2}-\boldsymbol{q}^{2}\right)^{2}\right]^{\frac{3}{2}}}\right\},
\end{aligned}
$$

where the second step is obtained after angular integration. Furthermore, under the assumption that $\alpha_{s}$ is not running, Eq. (3.1) can be further analytically integrated over $0 \leqslant|\boldsymbol{k}| \leqslant k_{\max }$, where $k_{\max }=2 E \sqrt{x(1-x)}$ [18]. We obtain

$$
\frac{\Delta E_{\mathrm{dyn}}}{E}=\frac{C_{R} \alpha_{s}}{\pi^{2}} \frac{L}{\lambda_{\mathrm{dyn}}} \int d x d^{2} q \mathcal{J}_{\mathrm{dyn}}(\boldsymbol{q}, x),
$$


where

$$
\begin{aligned}
& \mathcal{J}_{\text {dyn }}(\boldsymbol{q}, x) \\
& =\frac{\mu^{2}}{\boldsymbol{q}^{2}\left(\boldsymbol{q}^{2}+\mu^{2}\right)}\left\{-1+\frac{\boldsymbol{q}^{2}-k_{\max }^{2}+\chi}{\sqrt{\boldsymbol{q}^{4}+2 \boldsymbol{q}^{2}\left(\chi-k_{\max }^{2}\right)+\left(k_{\max }^{2}+\chi\right)^{2}}}+\ln \left[\frac{\chi+k_{\max }^{2}-\boldsymbol{q}^{2}+\sqrt{\boldsymbol{q}^{4}+2 \boldsymbol{q}^{2}\left(\chi-k_{\max }^{2}\right)+\left(k_{\max }^{2}+\chi\right)^{2}}}{2\left(\chi+k_{\max }^{2}\right)}\right]\right. \\
& \left.\quad+\frac{\left(\boldsymbol{q}^{2}+2 \chi\right)}{\boldsymbol{q}^{2} \sqrt{1+\frac{4 \chi}{\boldsymbol{q}^{2}}}} \ln \left[\frac{k_{\max }^{2}+\chi}{\chi} \frac{\left(\boldsymbol{q}^{2}-k_{\max }^{2}+3 \chi\right)+\sqrt{1+\frac{4 \chi}{\boldsymbol{q}^{2}}}\left(\boldsymbol{q}^{2}+\chi\right)}{\left(\boldsymbol{q}^{2}\right.} \sqrt{\boldsymbol{q}^{4}+2 \boldsymbol{q}^{2}\left(\chi-k_{\max }^{2}\right)+\left(k_{\max }^{2}+\chi\right)^{2}}\right]\right\}
\end{aligned}
$$

By comparing Eqs. (3.1)-(3.3) with the equivalent expressions from Ref. [8] [see Eqs. (2.9)-(2.12)], we see that, though similar, the corresponding expressions are not the same. The reason is that, in Ref. [8], we considered the Bethe-Heitler limit, which considers on-shell quark jets produced at remote past, while in our study, we allow that quark jets can be both on- and off-shell.

\section{LPM LIMIT}

The second relevant limit that we consider is the nonAbelian analog of the Landau-Pomeanchuk-Migdal limit. This limit is relevant for highly energetic jets, where destructive interference effects reduce the energy loss relative to the incoherent limit. The limit is obtained from the Eq. (2.12) when $E^{+} \approx 2 E \rightarrow \infty$. In such a limit finite mass effects are negligible. Additionally, $k_{\max }=2 E \sqrt{x(1-x)} \rightarrow \infty$ as well, which enables us to introduce a substitution $\boldsymbol{k}^{\prime} \equiv \boldsymbol{k}+\boldsymbol{q}$ in Eq. (2.12). With these simplifications, Eq. (2.12) becomes

$$
\begin{aligned}
\frac{\Delta E_{\mathrm{dyn}}}{E}= & \frac{C_{R} \alpha_{s}}{\pi} \frac{L}{\lambda_{\mathrm{dyn}}} \int d x \frac{d^{2} k^{\prime}}{\pi} \frac{d^{2} q}{\pi} \frac{\mu^{2}}{\boldsymbol{q}^{2}\left(\boldsymbol{q}^{2}+\mu^{2}\right)} \\
& \times \frac{2 \boldsymbol{q} \times\left(\boldsymbol{q}-\boldsymbol{k}^{\prime}\right)}{\boldsymbol{k}^{\prime 2}\left(\boldsymbol{k}^{\prime}-\boldsymbol{q}\right)^{2}}\left[1-\frac{\sin \left(\frac{\boldsymbol{k}^{\prime 2} L}{2 x E}\right)}{\left.\frac{\boldsymbol{k}^{\prime 2} L}{x E^{+}}\right]}\right. \\
= & 2 \frac{C_{R} \alpha_{s}}{\pi} \frac{L}{\lambda_{\mathrm{dyn}}} \int_{0}^{1} d x \int_{0}^{\infty} d \boldsymbol{k}^{\prime 2} \int_{0}^{q_{\max }^{2}} d \boldsymbol{q}^{2} \\
& \times \frac{\mu^{2}}{\boldsymbol{q}^{2}\left(\boldsymbol{q}^{2}+\mu^{2}\right)} \theta\left(|\boldsymbol{q}|-\left|\boldsymbol{k}^{\prime}\right|\right)\left[1-\frac{\sin \left(\frac{\boldsymbol{k}^{\prime 2} L}{2 x E}\right)}{\left.\frac{\boldsymbol{k}^{\prime 2} L}{2 x E}\right],}\right.
\end{aligned}
$$

where in the second step we performed angular integration. To proceed further, we observe that the derivative over distance $L$ of the above expression (i.e., $\frac{d E}{d L}$ ) is equal to

$$
\begin{aligned}
\frac{1}{E} \frac{d E_{\mathrm{dyn}}}{d L}= & 2 \frac{C_{R} \alpha_{s}}{\pi} \frac{1}{\lambda_{\mathrm{dyn}}} \int_{0}^{1} d x \int_{0}^{\infty} d \boldsymbol{k}^{\prime 2} \int_{0}^{q_{\max }^{2}} d \boldsymbol{q}^{2} \\
& \times \frac{\mu^{2}}{\boldsymbol{q}^{2}\left(\boldsymbol{q}^{2}+\mu^{2}\right)} \theta\left(|\boldsymbol{q}|-\left|\boldsymbol{k}^{\prime}\right|\right)\left[1-\cos \left(\frac{\boldsymbol{k}^{\prime 2} L}{2 x E}\right)\right] \\
= & 2 \frac{C_{R} \alpha_{s}}{\pi} \frac{1}{\lambda_{\mathrm{dyn}}} \int_{0}^{1} d x \int_{0}^{q_{\max }^{2}} d \boldsymbol{q}^{2} \frac{\mu^{2}}{\boldsymbol{q}^{2}\left(\boldsymbol{q}^{2}+\mu^{2}\right)} \\
& \times\left[\gamma-\mathrm{Ci}\left(\frac{L \boldsymbol{q}^{2}}{2 E x}\right)+\ln \left(\frac{L \boldsymbol{q}^{2}}{2 E x}\right)\right],
\end{aligned}
$$

where in the second step we performed an integral over $\boldsymbol{k}^{\prime 2}$, $\gamma \approx 0.577216$ is Euler's constant, and $\mathrm{Ci}(y)$ gives the cosine integral function.

Finally after performing the integration over $x$, we obtain

$$
\begin{aligned}
\frac{1}{E} \frac{d E_{\mathrm{dyn}}}{d L}= & 4 \frac{C_{R} \alpha_{s}}{\pi} \frac{1}{\lambda_{\mathrm{dyn}}} \int_{0}^{q_{\max }^{2}} d \boldsymbol{q}^{2} \frac{\mu^{2}}{\boldsymbol{q}^{2}\left(\boldsymbol{q}^{2}+\mu^{2}\right)} \\
& \times\left[Z\left(\frac{L \boldsymbol{q}^{2}}{2 E}\right)+\pi\left(\frac{L \boldsymbol{q}^{2}}{8 E}\right)\right],
\end{aligned}
$$

where

$$
\begin{aligned}
Z(y) \equiv & \gamma-\frac{1}{2} \cos (y)-C i(y)+\ln (y)+\frac{\sin (y)}{2 y} \\
& -\frac{y \operatorname{Si}(y)}{2} \underset{y \rightarrow 0}{\longrightarrow} 0 .
\end{aligned}
$$

Therefore, for the asymptotically large jet energies, the Eq. (4.3) reduces to

$$
\begin{aligned}
\frac{1}{E} \frac{d E_{\mathrm{dyn}}}{d L} & =\frac{C_{R} \alpha_{s}}{2 E} \frac{L}{\lambda_{\mathrm{dyn}}} \int_{0}^{q_{\max }^{2}} d \boldsymbol{q}^{2} \frac{\mu^{2}}{\boldsymbol{q}^{2}+\mu^{2}} \\
& =\frac{C_{R} \alpha_{s}}{2 E} \frac{L}{\lambda_{\mathrm{dyn}}} \mu^{2} \ln \left(\frac{4 E T}{\mu^{2}}\right),
\end{aligned}
$$

where we used $q_{\max }=\sqrt{4 E T}$ [39]. Finally, $\frac{\Delta E}{E}$ then becomes

$$
\frac{\Delta E_{\mathrm{dyn}}}{E}=\frac{C_{R} \alpha_{s}}{4 E} \frac{L^{2} \mu^{2}}{\lambda_{\mathrm{dyn}}} \ln \left(\frac{4 E T}{\mu^{2}}\right) .
$$

From the above expressions, we see that at asymptotically large jet energies we obtain quadratic thickness dependence for the energy loss, that is, we recover the LPM limit. Therefore, for highly energetic jet, finite-size corrections implemented in the calculation presented in this article simulate the destructive effects of LPM interference in an infinite QCD medium [5]. This behavior is expected [17] because the nuclear medium has finite dimensions that may be small compared to the jet radiation coherence length, especially in the case of light partons or high jet energies. Due to this, in finite-size media the basic formation time physics developed by LPM [12] leads to destructive interference effects on the quark quenching.

Furthermore, by applying the same procedure, it is straightforward to obtain that, for asymptotically large jet energies, the 
radiative energy loss in static QCD medium becomes

$$
\frac{\Delta E_{\text {stat }}}{E}=\frac{C_{R} \alpha_{s}}{4 E} \frac{L^{2} \mu^{2}}{\lambda_{\text {stat }}}\left[\ln \left(\frac{4 E T}{\mu^{2}}\right)-1\right],
$$

so that ratio between energy losses in dynamical and static QCD medium approaches

$$
\lim _{E \rightarrow \infty} \frac{\Delta E_{\text {dyn }}}{\Delta E_{\text {stat }}}=\lim _{E \rightarrow \infty} \frac{\lambda_{\text {stat }}}{\lambda_{\text {dyn }}} \frac{\ln \frac{4 E T}{\mu^{2}}}{\frac{4 E T}{\mu^{2}}-1}=\frac{\lambda_{\text {stat }}}{\lambda_{\text {dyn }}} .
$$

Therefore, we conclude that, at asymptotically large jet energies, approximation of the medium by a random distribution of static scatterers becomes valid, up to a multiplicative constant $\frac{\lambda_{\text {stat }}}{\lambda_{\text {dyn }}}$ that can be renormalized. This is consistent with what would be expected from established BDMPS results [5].

\section{CONCLUSION}

In this article, we developed a theoretical formalism for the calculation of the first order in opacity radiative energy loss of a fast quark traveling through a finite dynamical QCD medium. We obtain a closed analytical expression for the energy loss in dynamical medium. The obtained result is convergent, despite the fact that each individual contribution to the energy loss is infrared divergent. Furthermore, the energy loss has a nontrivial dependence on the size of the medium, which depends on both mass and energy of the quark jet. The finite-size effects are found to be most important in the ultrarelativistic limit and they effectively reproduce the effects of destructive LPM interference. Another interesting limit is an incoherent (GB) limit, which is reproduced for heavy quarks with moderately small jet energies.

The study presented here considered the radiative energy loss in finite-size dynamical QCD medium up to the first order in opacity. However, in static QCD medium, radiative energy loss up to all orders in opacity is obtained $[17,18]$. Simplicity of the mapping between the first order in opacity static and dynamical energy loss, implies that the same mapping might be generalized to higher order in opacity as well. That is, we make a conjecture that, to obtain the energy-loss expressions in dynamical QCD medium from the existing static QCD medium expressions, one (only) needs to replace effective mean free path and effective cross section from static QCD medium, with the corresponding expressions from dynamical QCD medium. However, to prove this conjecture is very nontrivial, which will be a subject of further research.

The measurement of the heavy-flavor suppression is in the current focus of intensive experimental efforts, and these measurements are expected to became available soon at the upcoming high-luminosity RHIC and LHC experiments. As already mentioned, particle suppression is a consequence of the energy loss. Our study, which incorporates dynamical effects in realistic finite-size QCD medium, enables us to provide the most reliable computations of the energy loss in QGP. Our future goal is to use these energy-loss calculations to make accurate theoretical predictions for the heavy-flavor suppression. These predictions can then be directly compared with the upcoming experimental data to test our understanding of QGP and to further study the properties of this novel form of matter.

\section{ACKNOWLEDGMENT}

Part of this work was done while M.D. was supported by the US Department of Energy, Grant DE-FG02-01ER41190.

\section{APPENDIX A: NOTATION AND ASSUMPTIONS}

In the following appendices, the calculation will mostly (with the exception of $G_{-+}$propagators), be done using the light cone coordinate system [40]. This coordinate system is appropriate for systems moving with almost the speed of light. It is obtained by choosing new spacetime coordinates $\left[x^{+}, x^{-}, \boldsymbol{x}\right]$, related to the coordinates in the laboratory frame $(t, z, \boldsymbol{x})$ by ( $\boldsymbol{x}$ is the transverse coordinate)

$$
x^{+}=(t+z), \quad x^{-}=(t-z) .
$$

In the same way the light cone momentum $\left[p^{+}, p^{-}, \boldsymbol{p}\right]$ is related to the momentum in the laboratory frame $\left[E, p_{z}, p\right]$ by ( $p$ is the transverse momentum)

$$
p^{+}=\left(E+p_{z}\right), \quad p^{-}=\left(E-p_{z}\right) .
$$

In this article we consider a quark of a finite mass $M$, produced inside the finite-size QGP, at some initial point $x_{0}$. The jet is assumed to have a large spatial momentum $p^{\prime} \gg M$, and it can be both on-shell and off-shell. This is in contrast to the Bethe-Heitler limit, which we considered in Ref. [8], where an on-shell jet was created at $-\infty$. Further, we choose coordinates such that the momentum of the initial quark is along the $z$ axis:

$$
p^{\prime}=\left[E^{\prime+}, p^{\prime-}, \mathbf{0}\right] .
$$

We are interested in the radiative energy loss to first order in the opacity, so we study the case in which the quark exchanges (in arbitrary sequence) one virtual gluon with spacelike momentum

$q=\left[q^{+}, q^{-}, \boldsymbol{q}\right]=\left(q_{0}, \overrightarrow{\mathbf{q}}\right)=\left(q_{0}, q_{z}, \boldsymbol{q}\right), \quad q_{0} \leqslant|\overrightarrow{\mathbf{q}}|$

with a parton in the medium and radiates one (mediummodified) real gluon with timelike momentum

$$
k=\left[k^{+}, k^{-}, \boldsymbol{k}\right]=\left(k_{0}, \overrightarrow{\mathbf{k}}\right)=\left(\omega, k_{z}, \boldsymbol{k}\right), \quad k_{0} \geqslant|\overrightarrow{\mathbf{k}}|
$$

into the medium. The on-shell quark jet emerges with fourmomentum $p^{\mu}$.

Similarly to all energy-loss formalisms developed so far (see e.g. Refs. [5,17,18,29-34]), the calculations presented in Appendices $\mathrm{C}-\mathrm{N}$ are developed under the assumption of a perturbative high-temperature QGP. We note that, strictly speaking, this assumption may not be directly applicable to the case of a strongly coupled QGP or in cold nuclear matter. The validity of this assumption for the QGP created in ultrarelativistic heavy-ion collisions can be tested by comparison with upcoming experimental data from the RHIC and LHC heavy-ion programs.

For the computation of the Feynman diagrams given in Appendices B-D we will need $G_{++}^{+}(x), G_{--}^{-}(x)$, and 
$G_{-+}(x)$ propagators for the quark jet $p$, the radiated gluon $k$, and the exchanged gluon $q$. These functions are derived in Appendix B.

As in Refs. [17,18,29-33], we assume validity of the soft-gluon $(\omega \ll E)$ and soft-rescattering $\left(|\boldsymbol{q}| \sim|\boldsymbol{k}| \ll k_{z}\right)$ approximations. These approximations become more reliable as the temperature increases and are expected to hold well at the LHC, while at RHIC their application should be further validated. Together with conservation of energy and momentum $\left(p^{\prime}=p+k+q\right)$ they yield

$$
\begin{aligned}
& k=\left[k^{+}, k^{-}=\frac{\boldsymbol{k}^{2}+m_{g}^{2}}{k^{+}}, \boldsymbol{k}\right], \\
& p=\left[E^{+}, p^{-}=\frac{\boldsymbol{p}^{2}+M^{2}}{E^{+}}, \boldsymbol{p}\right] .
\end{aligned}
$$

In Ref. [8] we showed that it is reasonable to assume that $q_{z}$ has the same order of magnitude as $|\boldsymbol{q}|$. Because $|\boldsymbol{k}| \ll k_{z}$ and $q_{z} \sim|\boldsymbol{q}| \sim|\boldsymbol{k}|$, we then also have $q_{z} \ll k_{z}$. Thus $k_{z}+q_{z} \approx k_{z}$ and $p_{z}+k_{z}+q_{z} \approx p_{z}+k_{z} \approx p_{z}+q_{z} \approx p_{z}$. Defining $x \equiv \frac{k^{+}}{E^{+}}$, we can therefore also assume that

$$
x \equiv \frac{k^{+}}{E^{+}} \approx \frac{(k+q)^{+}}{(E+q)^{+}} .
$$

By using $x$, we further define $\chi, \xi$, and $\zeta$ in the following way

$$
\begin{aligned}
\chi & \equiv M^{2} x^{2}+m_{g}^{2} \\
\xi & \equiv \frac{\boldsymbol{k}^{2}+\chi}{x E^{+}} \\
\zeta & \equiv \frac{(\boldsymbol{k}+\boldsymbol{q})^{2}+\chi}{x E^{+}} \\
\zeta-\xi & =\frac{(\boldsymbol{k}+\boldsymbol{q})^{2}-\boldsymbol{k}^{2}}{x E^{+}} .
\end{aligned}
$$

Finally, by using that $\xi, \zeta \ll|k| \sim|\boldsymbol{q}|$, and that $q_{z} \sim|\boldsymbol{q}| \sim|\boldsymbol{k}|$, we obtain $\xi, \zeta \ll q_{z}$ leading to

$$
\begin{aligned}
q^{-}+\xi & =q^{0}-\left(q_{z}-\xi\right) \approx q^{0}-q_{z} \\
q^{-}+\zeta & =q^{0}-\left(q_{z}-\zeta\right) \approx q^{0}-q_{z} \\
q^{-} \pm(\zeta-\xi) & =q^{0}-\left[q_{z} \pm(\xi-\zeta)\right] \approx q^{0}-q_{z} .
\end{aligned}
$$

\section{APPENDIX B: DERIVATION OF THE PROPAGATORS IN LIGHT CONE COORDINATE SYSTEM}

In this appendix we present in some detail the derivation of the propagators $G_{ \pm \pm}$in the light cone coordinate system, which are needed for the calculations presented in the following appendices. To derive these functions we start from Kallen-Lehmann theorem [41]:

$$
\begin{aligned}
G_{a b}(x)= & \frac{i}{2 \pi} \int_{0}^{\infty} d s \int \frac{d^{4} l}{(2 \pi)^{4}} \\
& \times\left[\frac{\rho_{a b}(s, l)}{l^{2}-s+i \epsilon}+\frac{\tilde{\rho}_{a b}(s, l)}{l^{2}-s-i \epsilon}\right] e^{-i l \cdot x},
\end{aligned}
$$

where $a, b$ can be + or - and $\rho_{a b}(s, l)$ and $\tilde{\rho}_{a b}(s, l)$ are spectral functions. We can now decompose the propagator as

$$
G_{a b}(x)=\theta\left(x^{+}\right) G_{a b}^{+}(x)+\theta\left(-x^{+}\right) G_{a b}^{-}(x) .
$$

Note that

$$
\begin{aligned}
l \cdot x & =\frac{1}{2} l^{+} x^{-}+\frac{1}{2} l^{-} x^{+}-\boldsymbol{l} \cdot \boldsymbol{x} \\
l^{2} & =l^{+} l^{-}-l^{2},
\end{aligned}
$$

We first concentrate on $G_{a b}^{+}(x)$. To obtain $G_{a b}^{+}(x)$ we take poles from Eq. (B1). Pole $l^{2}-s+i \epsilon=0$ contributes to $G_{a b}^{+}(x)$ only if $l^{+}>0$, while pole $l^{2}-s-i \epsilon=0$ contributes to $G_{a b}^{+}(x)$ only if $l^{+}<0$, leading to

$$
\begin{aligned}
G_{a b}^{+}(x) & \\
= & \int \frac{d^{4} l}{(2 \pi)^{4}} e^{-i l \cdot x} \int_{0}^{\infty} d s \delta\left(l^{2}-s\right)\left[\theta\left(l^{+}\right) \rho_{a b}(s, l)\right. \\
& \left.-\theta\left(-l^{+}\right) \tilde{\rho}_{a b}(s, l)\right] \\
= & \int \frac{d^{4} l}{(2 \pi)^{4}} e^{-i l \cdot x} G_{a b}^{+}(l),
\end{aligned}
$$

where

$$
G_{a b}^{+}(l)=\theta\left(l^{+}\right) \rho_{a b}\left(l^{2}, l\right)-\theta\left(-l^{+}\right) \tilde{\rho}_{a b}\left(l^{2}, l\right),
$$

where $\theta\left(l^{+}\right)$and $\theta\left(-l^{+}\right)$are unit step functions.

To proceed further, we use the following expressions

$$
\begin{aligned}
& \rho_{++}\left(l^{2}, l\right)=-\tilde{\rho}_{--}\left(l^{2}, l\right)=\left[\theta\left(l^{0}\right)+f\left(l^{0}\right)\right] \rho(l) \\
& \rho_{--}\left(l^{2}, l\right)=-\tilde{\rho}_{++}\left(l^{2}, l\right)=\left[\theta\left(-l^{0}\right)+f\left(l^{0}\right)\right] \rho(l) \\
& \rho_{+-}\left(l^{2}, l\right)=-\tilde{\rho}_{+-}\left(l^{2}, l\right)=f\left(l^{0}\right) \rho(l) \\
& \rho_{-+}\left(l^{2}, l\right)=-\tilde{\rho}_{-+}\left(l^{2}, l\right)=\left[1+f\left(l^{0}\right)\right] \rho(l),
\end{aligned}
$$

which can be straightforwardly derived from Ref. [41]. Here $\theta\left(l^{0}\right)$ is unit step function, $f\left(l^{0}\right)=\left(e^{l_{0} / T}-1\right)^{-1}$, where $T$ is the temperature of the medium and $\rho(l)$ is a spectral function.

Then, by using Eqs. (B6) and (B7), we obtain

$$
\begin{aligned}
G_{++}^{+}(l) & =-\theta\left(l^{+}\right) \rho_{++}(l)+\theta\left(-l^{+}\right) \rho_{--}(l) \\
& =\left[\theta\left(l^{+}\right) \theta\left(l^{0}\right)+\theta\left(-l^{+}\right) \theta\left(-l^{0}\right)+f\left(l^{0}\right)\right] \rho(l) \\
G_{--}^{+}(l) & =-\theta\left(l^{+}\right) \rho_{--}(l)+\theta\left(-l^{+}\right) \rho_{++}(l) \\
& =\left[\theta\left(l^{+}\right) \theta\left(-l^{0}\right)+\theta\left(-l^{+}\right) \theta\left(l^{0}\right)+f\left(l^{0}\right)\right] \rho(l) \\
G_{+-}^{+}(l) & =-\theta\left(l^{+}\right) \rho_{+-}(l)+\theta\left(-l^{+}\right) \rho_{+-}(l) \\
& =\rho_{+-}(l)=f\left(l^{0}\right) \rho(l) \\
G_{-+}^{+}(l) & =-\theta\left(l^{+}\right) \rho_{-+}(l)+\theta\left(-l^{+}\right) \rho_{-+}(l) \\
& =\rho_{-+}(l)=\left[1+f\left(l^{0}\right)\right] \rho(l) .
\end{aligned}
$$

Furthermore, it is straightforward to show that

$$
\begin{aligned}
& G_{++}^{+}(l)=G_{--}^{-}(l) \\
& G_{-+}^{-}(l)=G_{+-}^{+}(l) .
\end{aligned}
$$

In this article we will need only $G_{++}^{+}(x), G_{--}^{-}(x)$, and $G_{-+}(x)$ propagators for the quark jet $p$, the radiated gluon $k$, and the exchanged gluon $q$. We will first derive these functions for the exchanged gluon $q$. To do this, let us first note that, as noted in the previous appendix, in this article we assume high-temperature QGP and a soft-gluon, soft-rescattering approximation. In such a limit, for the exchanged gluon $q$, $f\left(q^{0}\right) \approx \frac{T}{q_{0}} \gg 1$, reducing the Eqs. (B8) and (B9) to

$$
\begin{aligned}
G_{++}^{+}(q) & \approx G_{--}^{+}(q) \approx G_{+-}^{+}(q) \approx G_{-+}^{+}(q) \approx G_{--}^{-}(q) \\
& \approx G_{-+}^{-}(q) \approx f\left(q^{0}\right) \rho(q) .
\end{aligned}
$$


By using Eq. (B10), it is then straightforward to obtain that corresponding propagators for the exchanged gluon are given by

$$
\begin{aligned}
D_{++}^{+\mu \nu}\left(x_{i}-x_{j}\right) & =D_{--}^{-\mu \nu}\left(x_{i}-x_{j}\right) \approx D_{+-}^{\mu \nu}\left(x_{i}-x_{j}\right) \\
& \approx \int \frac{d^{4} q}{(2 \pi)^{4}} D^{>}(q) e^{-i q\left(x_{i}-x_{j}\right)},
\end{aligned}
$$

where $D^{>}(q)$ is the effective 1-HTL cut gluon propagator for the exchanged gluon [8]

$$
\begin{aligned}
D_{\mu \nu}^{>}(q)= & \theta\left(1-\frac{q_{0}^{2}}{\overrightarrow{\mathbf{q}}^{2}}\right)\left[1+f\left(q_{0}\right)\right] \\
& \times 2 \operatorname{Im}\left[\frac{P_{\mu \nu}(q)}{q^{2}-\Pi_{T}(q)}+\frac{Q_{\mu \nu}(q)}{q^{2}-\Pi_{L}(q)}\right] .
\end{aligned}
$$

Here $\Pi_{T}(q)$ and $\Pi_{L}(q)$ [see Eq. (2.2)] are transverse and longitudinal self-energies. Note that exchanged gluons are spacelike [see Eq. (A4)]. Therefore, in the cut 1-HTL exchanged gluon propagator, only Landau damping contribution from the gluon spectral function contribute to the above expression (for more details, see Ref. [8]).

We will now concentrate on the propagators for the radiated gluon and quarks jet. For radiated gluon and quark jet $f\left(l_{0}\right)=$ $\left(e^{l_{0} / T}-1\right)^{-1} \ll 1$, reducing the Eq. (B8) to

$$
\begin{aligned}
& G_{++}^{+}(l)=\left[\theta\left(l^{+}\right) \theta\left(l^{0}\right)+\theta\left(-l^{+}\right) \theta\left(-l^{0}\right)\right] \rho(l) \\
& G_{--}^{+}(l)=\left[\theta\left(l^{+}\right) \theta\left(-l^{0}\right)+\theta\left(-l^{+}\right) \theta\left(l^{0}\right)\right] \rho(l) \\
& G_{+-}^{+}(l)=0 \\
& G_{-+}^{+}(l)=\rho(l) .
\end{aligned}
$$

To proceed further with propagators for the radiated gluon propagator, we note that, for the radiated gluon with momentum $k$, the longitudinal contribution can be neglected relative to the transverse one. Also, for the transverse gluon the selfenergy $\Pi_{T}(k)$ can be approximated by $m_{g}^{2}$, where $m_{g} \approx \mu / \sqrt{2}$ is the asymptotic mass (see Ref. [14]). These approximations are true in the soft-rescattering limit $\omega \gg|\boldsymbol{q}| \sim|\boldsymbol{k}| \sim g T$ that we use in this article. With these approximations the HTL gluon propagator for the emitted gluon can be simplified to [14]

$$
D_{\mu \nu}(k) \approx-i \frac{P_{\mu \nu}(k)}{k^{2}-m_{g}^{2}+i \epsilon},
$$

where $P_{\mu \nu}$ is the transverse projector.

Then, by using Eqs. (B13) and (B14), relevant radiative gluon propagators become (see also [40]):

$$
\begin{aligned}
D_{++}^{+\mu \nu}\left(x_{i}-x_{j}\right) & =D_{--}^{-\mu v}\left(x_{i}-x_{j}\right) \\
& =\int \frac{d k^{+} d^{2} k}{(2 \pi)^{3} 2 k^{+}} \theta\left(k^{+}\right) P^{\mu v}(k) e^{-i k\left(x_{i}-x_{j}\right)} \\
D_{-+}^{\mu v}\left(x_{i}-x_{j}\right) & =\int \frac{d^{3} k}{(2 \pi)^{3} 2 \omega} P^{\mu v}(k) e^{-i k\left(x_{i}-x_{j}\right)},
\end{aligned}
$$

where $\omega \approx \sqrt{\hat{\mathbf{k}}^{2}+m_{g}^{2}}$ and $k^{+}=\omega+k_{z}$.

Note that we here assume that gluon mass is given by the expression $m_{g} \approx \mu / \sqrt{2}$ regardless whether the gluon is radiated inside or outside of the medium. Strictly speaking, including the finite-size effects on the radiated gluon would assume that the above gluon mass is valid only for gluons radiated inside the medium, while for the gluons radiated outside the medium the gluon mass should be equal to zero (see Ref. [33]). However, based on [33], neglecting the finite-size effects on the radiated gluon is expected to be a reasonable approximation, and we adopt it here to simplify the calculations.

Finally, we now concentrate on the relevant propagators for the quark jet. By repeating similar procedure as for the radiated gluon, we obtain

$$
\begin{aligned}
\Delta_{++}^{+}\left(x_{i}-x_{j}\right) & =\Delta_{--}^{-}\left(x_{i}-x_{j}\right) \\
& =\int \frac{d p^{+} d^{2} p}{(2 \pi)^{3} 2 p^{+}} \theta\left(p^{+}\right) e^{-i p\left(x_{i}-x_{j}\right)} \\
\Delta_{-+}\left(x_{i}-x_{j}\right) & =\int \frac{d^{3} p}{(2 \pi)^{3} 2 E} e^{-i p\left(x_{i}-x_{j}\right)}
\end{aligned}
$$

\section{APPENDIX C: COMPUTATION OF DIAGRAM $M_{1,0,1, C}$}

In Appendices $\mathrm{C}-\mathrm{G}$ we present in some detail the calculation of the diagrams where both ends of the exchanged gluon $q$ are attached to the heavy quark, i.e., none is attached to the radiated gluon $k$ and no three-gluon vertex is involved. In this appendix, we start with the calculation of the diagram shown in Fig. 2.

Here and later the diagrams are labeled as follows: In $M_{1, i, j, C}, 1$ denotes that these diagrams contribute to the energy loss to first order in opacity, $i$ denotes how many ends of the virtual gluon $q$ are attached to the radiated gluon $k$, and $j$ labels the specific diagram in that class. The letter $C$ denotes that we consider central cut of the diagram. In the next chapters, letter $R(L)$ will denote that we consider right (left) cut of the Feynman diagram.

We will first calculate the cut diagram $M_{1,0,1, C}$ :

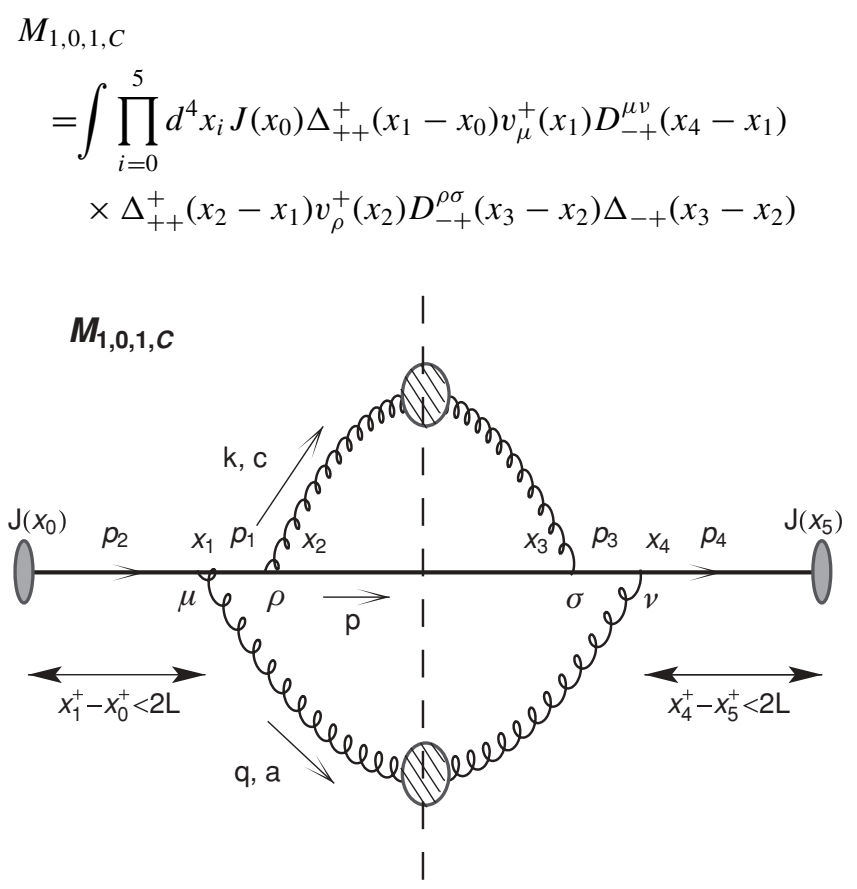

FIG. 2. Feynman diagram $M_{1,0,1, C}$, contributing to the radiative energy loss to first order in opacity, labeled the same way as in Fig. 1. 


$$
\begin{aligned}
& \times v_{\sigma}^{-}\left(x_{3}\right) \Delta_{--}^{-}\left(x_{4}-x_{3}\right) v_{v}^{-}\left(x_{4}\right) \Delta_{--}^{-}\left(x_{5}-x_{4}\right) J\left(x_{5}\right) \\
& \times \theta\left(x_{1}^{+}-x_{0}^{+}\right) \theta\left(x_{2}^{+}-x_{1}^{+}\right) \theta\left[2 L-\left(x_{1}^{+}-x_{0}^{+}\right)\right] \\
& \times \theta\left(x_{4}^{+}-x_{5}^{+}\right) \theta\left(x_{3}^{+}-x_{4}^{+}\right) \theta\left[2 L-\left(x_{4}^{+}-x_{5}^{+}\right)\right]
\end{aligned}
$$

where $J$ is the source of a jet, $\Delta$ correspond to the jet propagator, $D$ to gluon propagators and $v$ to vertices. By using expressions for the propagators from the previous section, we obtain:

$$
\begin{aligned}
& M_{1,0,1, C} \\
& =\int \prod_{i=0}^{5} d^{4} x_{i} J\left(x_{0}\right) \int_{-\infty}^{\infty} \int_{0}^{\infty} \frac{d p_{2}^{+} d^{2} p_{2}}{(2 \pi)^{3} 2 p_{2}^{+}} e^{-i p_{2} \cdot\left(x_{1}-x_{0}\right)} \\
& \times \int \frac{d^{4} q}{(2 \pi)^{4}} D_{\mu \nu}^{>}(q) e^{-i q\left(x_{4}-x_{1}\right)} \int_{-\infty}^{\infty} \int_{0}^{\infty} \frac{d p_{1}^{+} d^{2} p_{1}}{(2 \pi)^{3} 2 p_{1}^{+}} \\
& \times e^{-i p_{1} \cdot\left(x_{2}-x_{1}\right)}\left[-i g\left(p_{2}+p_{1}\right)^{\mu} t_{a}\right](-1) \int \frac{d^{3} k}{(2 \pi)^{3} 2 \omega} \\
& \times P_{\rho \sigma}(k) e^{-i k \cdot\left(x_{3}-x_{2}\right)} \int \frac{d^{3} p}{(2 \pi)^{3} 2 E} e^{-i p \cdot\left(x_{3}-x_{2}\right)} \\
& \times\left[-i g\left(p_{1}+p\right)^{\rho} t_{c}\right] \int_{-\infty}^{\infty} \int_{0}^{\infty} \frac{d p_{3}^{+} d^{2} p_{3}}{(2 \pi)^{3} 2 p_{3}^{+}} e^{-i p_{3} \cdot\left(x_{4}-x_{3}\right)} \\
& \times\left(i g\left(p+p_{3}\right)^{\sigma} t_{c}\right) \int_{-\infty}^{\infty} \int_{0}^{\infty} \frac{d p_{4}^{+} d^{2} p_{4}}{(2 \pi)^{3} 2 p_{4}^{+}} e^{-i p_{4} \cdot\left(x_{5}-x_{4}\right)} \\
& \times\left(i g\left(p_{3}+p_{4}\right)^{v} t_{a}\right) J\left(x_{5}\right) \theta\left(x_{1}^{+}-x_{0}^{+}\right) \\
& \times \theta\left(x_{2}^{+}-x_{1}^{+}\right) \theta\left[2 L-\left(x_{1}^{+}-x_{0}^{+}\right)\right] \theta\left(x_{4}^{+}-x_{5}^{+}\right) \\
& \times \theta\left(x_{3}^{+}-x_{4}^{+}\right) \theta\left(2 L-\left(x_{4}^{+}-x_{5}^{+}\right)\right) \\
& =\int_{-\infty}^{\infty} \int_{0}^{\infty} \prod_{i=1}^{4} \frac{d p_{i}^{+} d^{2} p_{i}}{(2 \pi)^{3} 2 p_{i}^{+}} \int \frac{d^{3} k}{(2 \pi)^{3} 2 \omega} \int \frac{d^{3} p}{(2 \pi)^{3} 2 E} \\
& \times \int \frac{d^{4} q}{(2 \pi)^{4}} g^{4} t_{a} t_{c} t_{c} t_{a}\left(p_{1}+p_{2}\right)^{\mu} D_{\mu \nu}^{>}(q) \\
& \times\left(p_{3}+p_{4}\right)^{\nu}\left(p+p_{1}\right)^{\rho} P_{\rho \sigma}(k)\left(p+p_{3}\right)^{\sigma} I,
\end{aligned}
$$

where

$$
\begin{aligned}
I= & \int \prod_{i=0}^{5} d^{4} x_{i} \theta\left(x_{1}^{+}-x_{0}^{+}\right) \theta\left(x_{2}^{+}-x_{1}^{+}\right) \theta\left[2 L-\left(x_{1}^{+}-x_{0}^{+}\right)\right] \\
& \times \theta\left(x_{4}^{+}-x_{5}^{+}\right) \theta\left(x_{3}^{+}-x_{4}^{+}\right) \theta\left[2 L-\left(x_{4}^{+}-x_{5}^{+}\right)\right] \\
& \times e^{-i p_{2} \cdot\left(x_{1}-x_{0}\right)} e^{-i q \cdot\left(x_{4}-x_{1}\right)} e^{-i p_{1} \cdot\left(x_{2}-x_{1}\right)} e^{-i(p+k) \cdot\left(x_{3}-x_{2}\right)} \\
& \times e^{-i p_{3} \cdot\left(x_{4}-x_{3}\right)} e^{-i p_{4} \cdot\left(x_{5}-x_{4}\right)} J\left(x_{0}\right) J\left(x_{5}\right) \\
= & |J(p)|^{2}(2 \pi)^{3} \delta\left[\left(p_{2}-p-k-q\right)^{+}\right] \delta^{2}\left(\boldsymbol{p}_{2}-\boldsymbol{p}-\boldsymbol{k}-\boldsymbol{q}\right) \\
& \times(2 \pi)^{3} \delta\left[\left(p_{1}-p-k\right)^{+}\right] \delta^{2}\left(\boldsymbol{p}_{1}-\boldsymbol{p}-\boldsymbol{k}\right)(2 \pi)^{3} \delta \\
& \times\left[\left(p_{3}-p-k\right)^{+}\right] \delta^{2}\left(\boldsymbol{p}_{3}-\boldsymbol{p}-\boldsymbol{k}\right)(2 \pi)^{3} \\
& \times \delta\left[\left(p_{4}-p-k-q\right)^{+}\right] \delta^{2}\left(\boldsymbol{p}_{4}-\boldsymbol{p}-\boldsymbol{k}-\boldsymbol{q}\right) I_{1}, \quad(\mathrm{C} 3)
\end{aligned}
$$

where

$$
\begin{aligned}
I_{1}= & \int_{0}^{\infty} d x_{2}^{\prime+} e^{-\frac{i}{2}\left(p_{1}-p-k\right)^{-} x_{2}^{\prime+}} \int_{0}^{2 L} d x_{1}^{\prime+} e^{-\frac{i}{2}\left(p_{2}-p-k-q\right)^{-} x_{1}^{\prime+}} \\
& \times \int_{0}^{\infty} d x_{3}^{\prime+} e^{\frac{i}{2}\left(p_{3}-p-k\right)^{-} x_{3}^{\prime+}} \int_{0}^{2 L} d x_{4}^{\prime+} e^{\frac{i}{2}\left(p_{4}-p-k-q\right)^{-} x_{4}^{\prime+}}
\end{aligned}
$$

Here we defined $x_{1}^{\prime}=x_{1}-x_{0}, x_{2}^{\prime}=x_{2}-x_{1}, x_{3}^{\prime}=x_{3}-x_{4}$, $x_{4}^{\prime}=x_{4}-x_{5}$.

By applying $\delta$ functions from Eq. (C3), and by using

$$
\begin{aligned}
& p_{i}^{-}=\frac{\boldsymbol{p}_{i}^{2}+M^{2}}{p_{i}^{+}} \\
& k^{-}=\frac{\boldsymbol{k}^{2}+m_{g}^{2}}{k^{+}},
\end{aligned}
$$

we obtain (note $\boldsymbol{p}+\boldsymbol{k}+\boldsymbol{q}=0 \rightarrow \boldsymbol{p}+\boldsymbol{k}=-\boldsymbol{q}$ ):

$$
p_{1}^{-}=p_{3}^{-}=\frac{q^{2}+M^{2}}{(p+k)^{+}} .
$$

Then in the soft-gluon, soft-rescattering limit we obtain [note $x \equiv \frac{k^{+}}{E^{+}}$and that $\xi$ and $\zeta$ are defined in Eq. (A8)]:

$$
\begin{aligned}
& \left(p_{1}-p-k\right)^{-}=\left(p_{3}-p-k\right)^{-}=\frac{\boldsymbol{k}^{2}+\chi}{x E^{+}}=-\xi \\
& \left(p_{2}-p-k\right)^{-}=\frac{(\boldsymbol{k}+\boldsymbol{q})^{2}+\chi}{x E^{+}}=-\zeta
\end{aligned}
$$

leading to

$$
I_{1}=\frac{16}{\left[\left(p_{1}-p-k\right)^{-}\right]^{2}} 4 \frac{\sin ^{2}\left[\left(p_{2}-p-k-q\right)^{-} \frac{L}{2}\right]}{\left[\left(p_{2}-p-k-q\right)^{-}\right]^{2}}
$$

In Ref. [13], it was shown that finite-size effects on collisions are negligible, i.e., for collisional parts

$$
\begin{aligned}
4 \frac{\sin ^{2}\left[\left(p_{2}-p-k-q\right)^{-} \frac{L}{2}\right]}{\left[\left(p_{2}-p-k-q\right)^{-}\right]^{2}} & \approx 2 \pi L \delta\left[\left(p_{2}-p-k-q\right)^{-}\right] \\
& \approx 2 \pi L \delta\left(q^{-}+\zeta\right) \\
& \approx 2 \pi L \delta\left(q^{0}-q_{z}\right),
\end{aligned}
$$

where in the last step we used soft-gluon, soft-rescattering approximation, i.e., Eq. (A9).

By using Eq. (C9), Eq. (C8) reduces to

$$
I_{1} \approx \frac{16 x^{2} E^{+2}}{\left(\boldsymbol{k}^{2}+\chi\right)^{2}} 2 \pi L \delta\left(q^{0}-q_{z}\right)
$$

Similarly as in Ref. [8], for highly energetic jets

$$
\begin{aligned}
\left(p+p_{1}\right)^{\rho} P_{\rho \sigma}(k)\left(p+p_{3}\right)^{\sigma} & \approx-4 \frac{\boldsymbol{k}^{2}}{x^{2}} \\
\left(p_{1}+p_{2}\right)^{\mu} P_{\mu \nu}(q)\left(p_{3}+p_{4}\right)^{v} & \approx-\left(p_{1}+p_{2}\right)^{\mu} Q_{\mu \nu}(q)\left(p_{3}+p_{4}\right)^{\nu} \\
& \approx-E^{+2} \frac{\boldsymbol{q}^{2}}{\overrightarrow{\mathbf{q}}^{2}}, \quad(\mathrm{C} 11)
\end{aligned}
$$

By using Eq. (C11) and Eqs. (B12), $\left(p_{1}+p_{2}\right)^{\mu} D_{\mu \nu}^{>}(q)\left(p_{3}+p_{4}\right)^{\nu}$ becomes

$$
\begin{aligned}
&\left(p_{1}+p_{2}\right)^{\mu} D_{\mu \nu}^{>}(q)\left(p_{3}+p_{4}\right)^{\nu} \\
& \approx\left(p_{1}+p_{2}\right)^{\mu} 2 \operatorname{Im}\left[\frac{P_{\mu \nu}(q)}{q^{2}-\Pi_{T}(q)}+\frac{Q_{\mu \nu}(q)}{q^{2}-\Pi_{L}(q)}\right]\left(p_{3}+p_{4}\right)^{\nu} \\
& \approx \theta\left(1-\frac{q_{0}^{2}}{\overrightarrow{\mathbf{q}}^{2}}\right) f\left(q_{0}\right) E^{+2} \frac{\boldsymbol{q}^{2}}{\overrightarrow{\mathbf{q}}^{2}} \\
& \quad \times 2 \operatorname{Im}\left[\frac{1}{q^{2}-\Pi_{L}(q)}-\frac{1}{q^{2}-\Pi_{T}(q)}\right]
\end{aligned}
$$


Finally, by using Eqs. (C3), (C4), (C10), (C11), and (C12), and after performing integrations over $p_{1}, p_{2}, p_{3}$, and $p_{4}$, Eq. (C2) reduces to

$$
\begin{aligned}
M_{1,0,1, C}= & 4 L g^{4} t_{a} t_{c} t_{c} t_{a} \int \frac{d^{3} p}{(2 \pi)^{3} 2 E}|J(p)|^{2} \\
& \times \frac{d^{3} k}{(2 \pi)^{3} 2 \omega} \frac{\boldsymbol{k}^{2}}{\left(\boldsymbol{k}^{2}+\chi\right)^{2}} I_{q},
\end{aligned}
$$

where

$$
\begin{aligned}
I_{q}= & \int \frac{d^{4} q}{(2 \pi)^{4}} 2 \pi \delta\left(q_{0}-q_{z}\right) f\left(q_{0}\right) \frac{\boldsymbol{q}^{2}}{\overrightarrow{\mathbf{q}}^{2}} \\
& \times 2 \operatorname{Im}\left[\frac{1}{q^{2}-\Pi_{L}(q)}-\frac{1}{q^{2}-\Pi_{T}(q)}\right] .
\end{aligned}
$$

In the high-temperature limit $I_{q}$ reduces to (see Appendix C in Ref. [8]):

$$
I_{q}=T \int \frac{d^{2} q}{(2 \pi)^{2}} \frac{\mu^{2}}{\boldsymbol{q}^{2}\left(\boldsymbol{q}^{2}+\mu^{2}\right)} .
$$

Finally, Eq. (C13) becomes

$$
\begin{aligned}
M_{1,0,1, C}= & 4 L T g^{4} t_{a} t_{c} t_{c} t_{a} \int \frac{d^{3} p}{(2 \pi)^{3} 2 E}|J(p)|^{2} \int \frac{d^{3} k}{(2 \pi)^{3} 2 \omega} \\
& \times \frac{d^{2} q}{(2 \pi)^{2}} \frac{\mu^{2}}{\boldsymbol{q}^{2}\left(\boldsymbol{q}^{2}+\mu^{2}\right)} \frac{\boldsymbol{k}^{2}}{\left(\boldsymbol{k}^{2}+\chi\right)^{2}} .
\end{aligned}
$$

\section{APPENDIX D: COMPUTATION OF DIAGRAMS}

$$
M_{1,0,2, C}, M_{1,0,2, R} \text { AND } M_{1,0,2, L}
$$

In this appendix we present in some detail the calculation of the diagrams shown in Fig. 3.

We will first calculate the cut diagram $M_{1,0,2, C}$, shown in the left panel of Fig. 3:

$$
\begin{aligned}
M_{1,0,2, C}= & \int \prod_{i=0}^{5} d^{4} x_{i} J\left(x_{0}\right) \Delta_{++}^{+}\left(x_{1}-x_{0}\right) v_{\mu}^{+}\left(x_{1}\right) D_{-+}^{\mu v}\left(x_{4}-x_{1}\right) \\
& \times \Delta_{++}^{+}\left(x_{2}-x_{1}\right) v_{\rho}^{+}\left(x_{2}\right) D_{-+}^{\rho \sigma}\left(x_{3}-x_{2}\right) \Delta_{-+}\left(x_{3}-x_{2}\right) \\
& \times v_{\sigma}^{-}\left(x_{3}\right) \Delta_{--}^{-}\left(x_{4}-x_{3}\right) v_{v}^{-}\left(x_{4}\right) \Delta_{--}^{-}\left(x_{5}-x_{4}\right) J\left(x_{5}\right) \\
& \times \theta\left(x_{1}^{+}-x_{0}^{+}\right) \theta\left(x_{2}^{+}-x_{1}^{+}\right) \theta\left[2 L-\left(x_{2}^{+}-x_{0}^{+}\right)\right] \\
& \times \theta\left(x_{4}^{+}-x_{5}^{+}\right) \theta\left(x_{3}^{+}-x_{4}^{+}\right) \theta\left[2 L-\left(x_{3}^{+}-x_{5}^{+}\right)\right]
\end{aligned}
$$

$$
\begin{aligned}
= & -\int_{-\infty}^{\infty} \int_{0}^{\infty} \prod_{i=1}^{4} \frac{d p_{i}^{+} d^{2} p_{i}}{(2 \pi)^{3} 2 p_{i}^{+}} \int \frac{d^{3} k}{(2 \pi)^{3} 2 \omega} \\
& \times \int \frac{d^{3} p}{(2 \pi)^{3} 2 E} \int \frac{d^{4} q}{(2 \pi)^{4}} g^{4} t_{c} t_{a} t_{a} t_{c}\left(p_{1}+p_{2}\right)^{\mu} \\
& \times P_{\mu \nu}(k)\left(p_{3}+p_{4}\right)^{\nu}\left(p+p_{1}\right)^{\rho} D_{\rho \sigma}^{>}(q)\left(p+p_{3}\right)^{\sigma} I,
\end{aligned}
$$

where

$$
\begin{aligned}
I= & \int \prod_{i=0}^{5} d^{4} x_{i} \theta\left(x_{1}^{+}-x_{0}^{+}\right) \theta\left(x_{2}^{+}-x_{1}^{+}\right) \theta\left[2 L-\left(x_{2}^{+}-x_{0}^{+}\right)\right] \\
& \times \theta\left(x_{4}^{+}-x_{5}^{+}\right) \theta\left(x_{3}^{+}-x_{4}^{+}\right) \theta\left[2 L-\left(x_{3}^{+}-x_{5}^{+}\right)\right] \\
& \times e^{-i p_{2} \cdot\left(x_{1}-x_{0}\right)} e^{-i k \cdot\left(x_{4}-x_{1}\right)} e^{-i p_{1} \cdot\left(x_{2}-x_{1}\right)} e^{-i(p+q) \cdot\left(x_{3}-x_{2}\right)} \\
& \times e^{-i p_{3} \cdot\left(x_{4}-x_{3}\right)} e^{-i p_{4} \cdot\left(x_{5}-x_{4}\right)} J\left(x_{0}\right) J\left(x_{5}\right) \\
= & |J(p)|^{2}(2 \pi)^{3} \delta\left[\left(p_{2}-p_{1}-k\right)^{+}\right] \delta^{2}\left(\boldsymbol{p}_{2}-\boldsymbol{p}_{1}-\boldsymbol{k}\right)(2 \pi)^{3} \\
& \times \delta\left[\left(p_{1}-p-q\right)^{+}\right] \delta^{2}\left(\boldsymbol{p}_{1}-\boldsymbol{p}-\boldsymbol{q}\right) \\
& \times(2 \pi)^{3} \delta\left[\left(p_{3}-p-q\right)^{+}\right] \delta^{2}\left(\boldsymbol{p}_{3}-\boldsymbol{p}-\boldsymbol{q}\right)(2 \pi)^{3} \\
& \times \delta\left[\left(p_{4}-p_{3}-k\right)^{+}\right] \delta^{2}\left(\boldsymbol{p}_{4}-\boldsymbol{p}_{3}-\boldsymbol{k}\right) I_{1}
\end{aligned}
$$

and where

$$
\begin{aligned}
I_{1}= & \int_{0}^{2 L} d x_{2}^{\prime+} e^{-\frac{i}{2}\left(p_{1}-p-q\right)^{-} x_{2}^{++}} \int_{0}^{x_{2}^{\prime+}} d x_{1}^{\prime+} e^{-\frac{i}{2}\left(p_{2}-p_{1}-k\right)^{-} x_{1}^{\prime+}} \\
& \times \int_{0}^{2 L} d x_{3}^{\prime+} e^{\frac{i}{2}\left(p_{3}-p-q\right)^{-} x_{3}^{\prime+}} \int_{0}^{x_{3}^{\prime+}} d x_{4}^{\prime+} e^{\frac{i}{2}\left(p_{4}-p_{3}-k\right)^{-} x_{4}^{\prime+}}
\end{aligned}
$$

Here we defined $x_{1}^{\prime}=x_{1}-x_{0}, x_{2}^{\prime}=x_{2}-x_{0}, x_{3}^{\prime}=x_{3}-x_{5}$, $x_{4}^{\prime}=x_{4}-x_{5}$.

By applying $\delta$ functions from Eq. (D2), and by using Eq. (C5), we obtain $p_{1}^{-}=p_{3}^{-}$and $p_{2}^{-}=p_{4}^{-}$.

$I_{1}$ then becomes

$$
\begin{aligned}
I_{1}= & \frac{16}{\left.\left[p_{2}-p_{1}-k\right)^{-}\right]^{2}}\left\{\frac{e^{-i\left(p_{2}-p-k-q\right)^{-} L}-1}{\left(p_{2}-p-k-q\right)^{-}}\right. \\
& \times\left[\frac{e^{i\left(p_{2}-p-k-q\right)^{-} L}-1}{\left(p_{2}-p-k-q\right)^{-}}-\frac{e^{i\left(p_{1}-p-q\right)^{-} L}-1}{\left(p_{1}-p-q\right)^{-}}\right]
\end{aligned}
$$

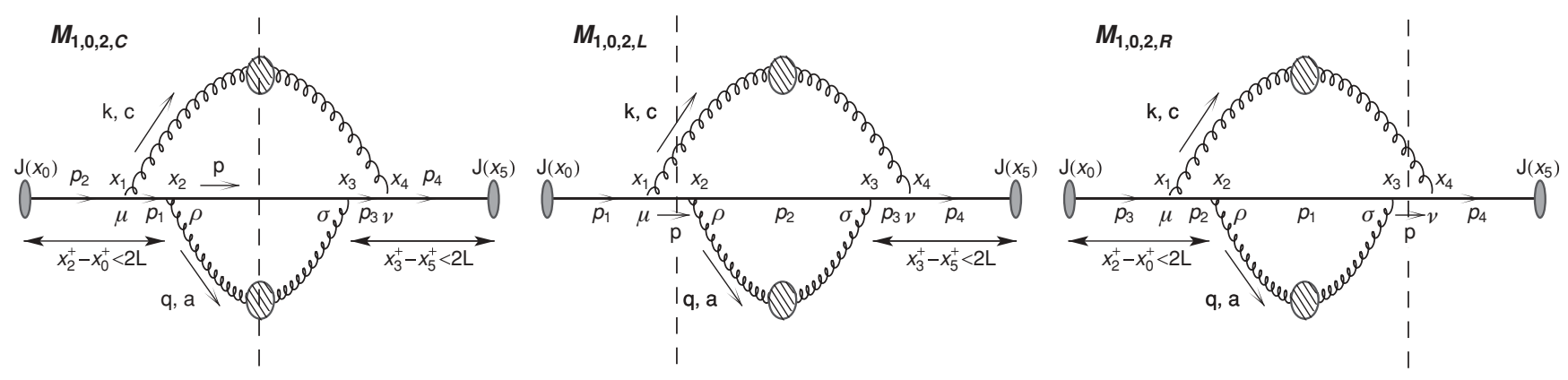

FIG. 3. Feynman diagrams $M_{1,0,2, C}, M_{1,0,2, L}$, and $M_{1,0,2, R}$ contributing to the radiative energy loss to first order in opacity, labeled the same way as in Fig. 1. The same figure is also presented as Fig. 1 in the main text and is repeated here for completeness. 


$$
\begin{aligned}
& +\frac{e^{-i\left(p_{1}-p-q\right)^{-} L}-1}{\left(p_{1}-p-q\right)^{-}}\left[\frac{e^{i\left(p_{2}-p-k-q\right)^{-} L}-1}{\left(p_{2}-p-k-q\right)^{-}}\right. \\
& \left.\left.-\frac{e^{i\left(p_{1}-p-q\right)^{-} L}-1}{\left(p_{1}-p-q\right)^{-}}\right]\right\} \\
& =\frac{16}{\left[\left(p_{2}-p_{1}-k\right)^{-}\right]^{2}}\left\{4 \frac{\sin ^{2}\left[\left(p_{2}-p-k-q\right)^{-} \frac{L}{2}\right]}{\left[\left(p_{2}-p-k-q\right)^{-}\right]^{2}}\right. \\
& \times\left[1-\frac{\left(p_{2}-p-k-q\right)^{-}}{e^{i\left(p_{2}-p-k-q\right)^{-} L}-1} \frac{e^{i\left(p_{1}-p-q\right)^{-} L}-1}{\left(p_{1}-p-q\right)^{-}}\right] \\
& +4 \frac{\sin ^{2}\left[\left(p_{1}-p-q\right)^{-}-\frac{L}{2}\right]}{\left[\left(p_{1}-p-q\right)^{-}\right]^{2}} \\
& \left.\times\left[1-\frac{e^{i\left(p_{1}-p-q\right)^{-} L}-1}{\left(p_{1}-p-q\right)^{-}} \frac{\left(p_{2}-p-k-q\right)^{-}}{e^{i\left(p_{2}-p-k-q\right)^{-} L}-1}\right]\right\} .
\end{aligned}
$$

In Ref. [13], it was shown that finite-size effect are small for collisional energy loss, i.e., for collisional parts the following approximation can be used

$$
\begin{aligned}
4 \frac{\sin ^{2}\left[\left(p_{2}-p-k-q\right)^{-} \frac{L}{2}\right]}{\left[\left(p_{2}-p-k-q\right)^{-}\right]^{2}} & \approx 2 \pi L \delta\left[\left(p_{2}-p-k-q\right)^{-}\right] \\
\frac{\sin ^{2}\left[\left(p_{1}-p-q\right)^{-} \frac{L}{2}\right]}{\left[\left(p_{1}-p-q\right)^{-}\right]^{2}} & \approx 2 \pi L \delta\left[\left(p_{1}-p-q\right)^{-}\right] .
\end{aligned}
$$

By using Eq. (D5), Eq. (D4) reduces to

$$
\begin{aligned}
I_{1}= & \frac{32 \pi L}{\left[\left(p_{1}+k-p_{2}\right)^{-}\right]^{2}}\left\{\delta\left[\left(p_{2}-p-k-q\right)^{-}\right]\right. \\
& \times\left[1-\frac{e^{i\left(p_{1}+k-p_{2}\right)^{-} L}-1}{i\left(p_{1}+k-p_{2}\right)^{-} L}\right] \delta\left[\left(p_{1}-p-q\right)^{-}\right] \\
& \left.+\left[1-\frac{e^{-i\left(p_{1}+k-p_{2}\right)^{-} L}-1}{-i\left(p_{1}+k-p_{2}\right)^{-} L}\right]\right\} \\
= & \frac{32 \pi L}{\left[\left(p_{1}+k-p_{2}\right)^{-}\right]^{2}}\left(\left\{\delta\left[\left(p_{2}-p-k-q\right)^{-}\right]\right.\right. \\
& \left.+\delta\left[\left(p_{1}-p-q\right)^{-}\right]\right\}\left\{1-\frac{\sin \left[\left(p_{1}+k-p_{2}\right)^{-} L\right]}{\left(p_{1}+k-p_{2}\right)^{-} L}\right\} \\
& +i\left\{\delta\left[\left(p_{2}-p-k-q\right)^{-}\right]-\delta\left[\left(p_{1}-p-q\right)^{-}\right\}\right) \\
& \left.\times \frac{\cos \left[\left(p_{1}+k-p_{2}\right)^{-} L\right]-1}{\left(p_{1}+k-p_{2}\right)^{-} L}\right) .
\end{aligned}
$$

In the soft-gluon, soft-rescattering approximation

$$
\begin{aligned}
\left(p_{1}-p\right)^{-} & \approx \frac{\boldsymbol{k}^{2}-(\boldsymbol{k}+\boldsymbol{q})^{2}}{E^{+}} \\
\left(p_{1}+k-p_{2}\right)^{-} & \approx \xi .
\end{aligned}
$$

Note that $\left(p_{1}-p\right)^{-} \ll \xi \ll|\boldsymbol{k}|,|\boldsymbol{q}|, q_{z}$, leading to

$$
\begin{gathered}
\left\{\delta\left[\left(p_{2}-p-k-q\right)^{-}\right]+\delta\left[\left(p_{1}-p-q\right)^{-}\right]\right\} \\
\approx 2 \delta\left[\left(p_{2}-p-k-q\right)^{-}\right] \approx 2 \delta\left(q_{0}-q_{z}\right), \\
\left\{\delta\left[\left(p_{2}-p-k-q\right)^{-}\right]-\delta\left[\left(p_{1}-p-q\right)^{-}\right]\right\} \\
\approx \delta\left(q_{0}-q_{z}+\xi\right)-\delta\left(q_{0}-q_{z}\right) .
\end{gathered}
$$

By using Eq. (D8), Eq. (D6) finally reduces to

$$
\begin{aligned}
I_{1} \approx & 2 \pi L \frac{16 x^{2} E^{+2}}{\left(\boldsymbol{k}^{2}+\chi\right)^{2}}\left\{2 \delta\left(q^{0}-q_{z}\right)\left[1-\frac{\sin (\xi L)}{\xi L}\right]\right. \\
& \left.+i\left[\delta\left(q_{0}-q_{z}+\xi\right)-\delta\left(q_{0}-q_{z}\right)\right] \frac{\cos (\xi L)-1}{\xi L}\right\} .
\end{aligned}
$$

Similarly as in previous section, for highly energetic jets and in a high-temperature limit

$$
\begin{gathered}
\left(p_{1}+p_{2}\right)^{\mu} P_{\mu \nu}(k)\left(p_{3}+p_{4}\right)^{v} \approx-4 \frac{\boldsymbol{k}^{2}}{x^{2}}, \\
\left(p+p_{1}\right)^{\rho} D_{\rho \sigma}^{>}(q)\left(p+p_{3}\right)^{\sigma} \\
\approx \theta\left(1-\frac{q_{0}^{2}}{\overrightarrow{\mathbf{q}}^{2}}\right) \frac{T}{q_{0}} E^{+2} \frac{\boldsymbol{q}^{2}}{\overrightarrow{\mathbf{q}}^{2}} 2 \operatorname{Im}\left[\frac{1}{q^{2}-\Pi_{L}(q)}-\frac{1}{q^{2}-\Pi_{T}(q)}\right] \\
\equiv F\left(q_{0}, q_{z}, \boldsymbol{q}\right)
\end{gathered}
$$

By using Eqs. (D2), (D9), and (D10), and after performing integrations over $p_{1}, p_{2}, p_{3}$, and $p_{4}$, Eq. (D1) becomes

$M_{1,0,2, C}$

$$
\begin{aligned}
= & 4 L T g^{4} t_{c} t_{a} t_{a} t_{c} \int \frac{d^{3} p}{(2 \pi)^{3} 2 E}|J(p)|^{2} \frac{d^{3} k}{(2 \pi)^{3} 2 \omega} \frac{\boldsymbol{k}^{2}}{\left(\boldsymbol{k}^{2}+\chi\right)^{2}} \\
& \times\left\{2\left[1-\frac{\sin (\xi L)}{\xi L}\right] I_{q}+i \frac{\cos (\xi L)-1}{\xi L} J_{q}\right\}, \quad(\mathrm{D} 11)
\end{aligned}
$$

where $I_{q}$ is given by Eq. (C15) and

$$
\begin{aligned}
J_{q} & =\int \frac{d^{4} q}{(2 \pi)^{4}}\left[\delta\left(q_{0}-q_{z}+\xi\right)-\delta\left(q_{0}-q_{z}\right)\right] F\left(q_{0}, q_{z}, \boldsymbol{q}\right) \\
& =\left.\xi \int \frac{d^{3} q}{(2 \pi)^{3}} \frac{d F\left(q_{0}, q_{z}, \boldsymbol{q}\right)}{d q_{0}}\right|_{q_{0}=q_{z}}=0 .
\end{aligned}
$$

In the last step we used that $\left.\frac{d F\left(q_{0}, q_{z}, \boldsymbol{q}\right)}{d q_{0}}\right|_{q_{0}=q_{z}}$ is an odd function of $q_{z}$.

Finally, by using Eqs. (C15) and (D12), Eq. (D11) reduces to

$$
\begin{aligned}
M_{1,0,2, C}= & 8 L T g^{4} t_{c} t_{a} t_{a} t_{c} \int \frac{d^{3} p}{(2 \pi)^{3} 2 E}|J(p)|^{2} \\
& \times \int \frac{d^{3} k}{(2 \pi)^{3} 2 \omega} \frac{d^{2} q}{(2 \pi)^{2}} \frac{\mu^{2}}{\boldsymbol{q}^{2}\left(\boldsymbol{q}^{2}+\mu^{2}\right)} \frac{\boldsymbol{k}^{2}}{\left(\boldsymbol{k}^{2}+\chi\right)^{2}} \\
& \times\left[1-\frac{\sin (\xi L)}{\xi L}\right] .
\end{aligned}
$$

We will now calculate the cut diagrams $M_{1,0,2, L}$ and $M_{1,0,2, R}$, shown in the central and right panels of Fig. 3, respectively. We start with $M_{1,0,2, R}$ :

$$
\begin{aligned}
M_{1,0,2, R}= & \int \prod_{i=0}^{5} d^{4} x_{i} J\left(x_{0}\right) \Delta_{++}^{+}\left(x_{1}-x_{0}\right) v_{\mu}^{+}\left(x_{1}\right) \\
& \times D_{-+}^{\mu v}\left(x_{4}-x_{1}\right) \Delta_{++}^{+}\left(x_{2}-x_{1}\right) v_{\rho}^{+}\left(x_{2}\right) \\
& \times D_{++}^{+\rho \sigma}\left(x_{3}-x_{2}\right) \Delta_{++}^{+}\left(x_{3}-x_{2}\right) v_{\sigma}^{+}\left(x_{3}\right) \\
& \times \Delta_{-+}\left(x_{4}-x_{3}\right) v_{v}^{-}\left(x_{4}\right) \Delta_{--}^{-}\left(x_{5}-x_{4}\right) J\left(x_{5}\right) \\
& \times \theta\left(x_{1}^{+}-x_{0}^{+}\right) \theta\left(x_{2}^{+}-x_{1}^{+}\right) \theta\left(x_{3}^{+}-x_{2}^{+}\right) \\
& \times \theta\left[2 L-\left(x_{2}^{+}-x_{0}^{+}\right)\right] \theta\left(x_{4}^{+}-x_{5}^{+}\right)
\end{aligned}
$$




$$
\begin{aligned}
= & \int_{-\infty}^{\infty} \int_{0}^{\infty} \prod_{i=1}^{4} \frac{d p_{i}^{+} d^{2} p_{i}}{(2 \pi)^{3} 2 p_{i}^{+}} \int \frac{d^{3} k}{(2 \pi)^{3} 2 \omega} \int \frac{d^{3} p}{(2 \pi)^{3} 2 E} \\
& \times \int \frac{d^{4} q}{(2 \pi)^{4}} g^{4} t_{c} t_{a} t_{a} t_{c}\left(p_{2}+p_{3}\right)^{\mu} P_{\mu \nu}(k)\left(p_{+} p_{4}\right)^{v} \\
& \times\left(p_{1}+p_{2}\right)^{\rho} D_{\rho \sigma}^{>}(q)\left(p+p_{1}\right)^{\sigma} I,
\end{aligned}
$$

where

$$
\begin{aligned}
I= & \int \prod_{i=0}^{5} d^{4} x_{i} \theta\left(x_{1}^{+}-x_{0}^{+}\right) \theta\left(x_{2}^{+}-x_{1}^{+}\right) \theta\left(x_{3}^{+}-x_{2}^{+}\right) \\
& \times \theta\left[2 L-\left(x_{2}^{+}-x_{0}^{+}\right)\right] \theta\left(x_{4}^{+}-x_{5}^{+}\right) \\
& \times e^{-i p_{3} \cdot\left(x_{1}-x_{0}\right)} e^{-i k \cdot\left(x_{4}-x_{1}\right)} e^{-i p_{2} \cdot\left(x_{2}-x_{1}\right)} e^{-i\left(p_{1}+q\right) \cdot\left(x_{3}-x_{2}\right)} \\
& \times e^{-i p \cdot\left(x_{4}-x_{3}\right)} e^{-i p_{4} \cdot\left(x_{5}-x_{4}\right)} J\left(x_{0}\right) J\left(x_{5}\right) \\
= & |J(p)|^{2}(2 \pi)^{3} \delta\left[\left(p_{3}-p_{2}-k\right)^{+}\right] \delta^{2}\left(\boldsymbol{p}_{3}-\boldsymbol{p}_{2}-\boldsymbol{k}\right)(2 \pi)^{3} \\
& \times \delta\left[\left(p_{2}-p\right)^{+}\right] \delta^{2}\left(\boldsymbol{p}_{2}-\boldsymbol{p}\right) \\
& \times(2 \pi)^{3} \delta\left[\left(p_{1}-p+q\right)^{+}\right] \delta^{2}\left(\boldsymbol{p}_{1}-\boldsymbol{p}+\boldsymbol{q}\right)(2 \pi)^{3} \\
& \times \delta\left[\left(p_{4}-p-k\right)^{+}\right] \delta^{2}\left(\boldsymbol{p}_{4}-\boldsymbol{p}-\boldsymbol{k}\right) I_{1}
\end{aligned}
$$

and where

$$
\begin{aligned}
I_{1}= & \int_{0}^{2 L} d x_{2}^{\prime+} e^{-\frac{i}{2}\left(p_{2}-p\right)^{-} x_{2}^{\prime+}} \int_{0}^{x_{2}^{\prime+}} d x_{1}^{\prime+} e^{-\frac{i}{2}\left(p_{3}-p_{2}-k\right)^{-} x_{1}^{\prime+}} \\
& \times \int_{0}^{\infty} d x_{3}^{\prime+} e^{-\frac{i}{2}\left(p_{1}-p+q\right)^{-} x_{3}^{\prime+}} \int_{0}^{\infty} d x_{4}^{\prime+} e^{\frac{i}{2}\left(p_{4}-p-k\right)^{-} x_{4}^{\prime+}}
\end{aligned}
$$

Here we defined $x_{1}^{\prime}=x_{1}-x_{0}, x_{2}^{\prime}=x_{2}-x_{0}, x_{3}^{\prime}=x_{3}-x_{2}$, $x_{4}^{\prime}=x_{4}-x_{5}$.

By applying $\delta$ functions from Eq. (D15), and by using Eq. (C5), we obtain

$$
\begin{aligned}
p_{2}^{-}=p^{-} & \rightarrow\left(p_{2}-p\right)^{-}=0, \\
p_{3}^{-}=p_{4}^{-} & =\frac{(\boldsymbol{p}+\boldsymbol{k})^{2}+M^{2}}{(p+k)^{+}} \approx \frac{M^{2}}{E^{+}} \\
\left(p+k-p_{3}\right)^{-} & =\xi \\
\left(p_{1}-p+q\right)^{-} & \approx q^{-} .
\end{aligned}
$$

Note that in the second equation we used $\boldsymbol{p}=-\boldsymbol{k}$ and $(p+$ $k)^{+} \approx E^{+}$.

$I_{1}$ then becomes

$$
\begin{aligned}
I_{1}= & \int_{0}^{2 L} d x_{2}^{\prime+} \int_{0}^{x_{2}^{\prime+}} d x_{1}^{\prime+} e^{\frac{i}{2} \xi x_{1}^{\prime+}} \int_{0}^{\infty} d x_{3}^{\prime+} e^{-\frac{i}{2}\left(p_{1}-p+q\right)^{-} x_{2}^{\prime+}} \\
& \times \int_{0}^{\infty} d x_{4}^{\prime+} e^{-\frac{i}{2} \xi x_{4}^{\prime+}} \\
= & \frac{8 L}{\xi^{2}}\left(1-\frac{\sin \xi L}{\xi L}-i \frac{1-\cos \xi L}{\xi L}\right) \int_{0}^{\infty} d x_{3}^{\prime+} e^{-\frac{i}{2} q^{-} x_{3}^{\prime+}}
\end{aligned}
$$

(D18)
By using Eqs. (D18), (D15), and (D14) $M_{1,0,2, R}$ becomes $\left(y \equiv \frac{x_{3}^{\prime+}}{2}\right)$ :

$$
\begin{aligned}
M_{1,0,2, R}= & -4 L g^{4} t_{c} t_{a} t_{a} t_{c} \int \frac{d^{3} p}{(2 \pi)^{3} 2 E}|J(p)|^{2} \\
& \times \int \frac{d^{3} k}{(2 \pi)^{3} 2 \omega} \frac{\boldsymbol{k}^{2}}{\left(\boldsymbol{k}^{2}+\chi\right)^{2}} \\
& \times\left(1-\frac{\sin \xi L}{\xi L}-i \frac{1-\cos \xi L}{\xi L}\right) \\
& \times \int \frac{d^{4} q}{(2 \pi)^{4}}\left(p+p_{1}\right)^{\rho} D_{\rho \sigma}^{>}(q)\left(p+p_{1}\right)^{\sigma} \\
& \times \int_{0}^{\infty} d y e^{-i q^{-} y},
\end{aligned}
$$

where, as in the previous sections, we used $\left(p+p_{3}\right)^{\mu} P_{\mu \nu}(k)\left(p+p_{3}\right)^{v} \approx-4 \frac{k^{2}}{x^{2}}$.

In the same way, it can be obtained that $M_{1,0,2, L}$ is equal to

$$
\begin{aligned}
M_{1,0,2, L}= & -4 L g^{4} t_{c} t_{a} t_{a} t_{c} \int \frac{d^{3} p}{(2 \pi)^{3} 2 E}|J(p)|^{2} \\
& \times \int \frac{d^{3} k}{(2 \pi)^{3} 2 \omega} \frac{\boldsymbol{k}^{2}}{\left(\boldsymbol{k}^{2}+\chi\right)^{2}} \\
& \times\left(1-\frac{\sin \xi L}{\xi L}-i \frac{1-\cos \xi L}{\xi L}\right) \\
& \times \int \frac{d^{4} q}{(2 \pi)^{4}}\left(p+p_{1}\right)^{\rho} D_{\rho \sigma}^{>}(q)\left(p+p_{1}\right)^{\sigma} \\
& \times \int_{0}^{\infty} d y e^{i q^{-} y} .
\end{aligned}
$$

$M_{1,0,2, R}+M_{1,0,2, L}$ then becomes

$$
\begin{aligned}
M_{1,0,2, R}+M_{1,0,2, L}= & -4 L g^{4} t_{c} t_{a} t_{a} t_{c} \int \frac{d^{3} p}{(2 \pi)^{3} 2 E}|J(p)|^{2} \\
& \times \int \frac{d^{3} k}{(2 \pi)^{3} 2 \omega} \frac{\boldsymbol{k}^{2}}{\left(\boldsymbol{k}^{2}+\chi\right)^{2}} \int \frac{d^{4} q}{(2 \pi)^{4}} \\
& \times\left(p+p_{1}\right)^{\rho} D_{\rho \sigma}^{>}(q)\left(p+p_{1}\right)^{\sigma} I_{2},
\end{aligned}
$$

where

$$
\begin{aligned}
I_{2}= & \left(1-\frac{\sin \xi L}{\xi L}-i \frac{1-\cos \xi L}{\xi L}\right) \int_{0}^{\infty} d y e^{-i y q^{-}} \\
& +\left(1-\frac{\sin \xi L}{\xi L}+i \frac{1-\cos \xi L}{\xi L}\right) \int_{0}^{\infty} d y e^{i y q^{-}} \\
= & \left(1-\frac{\sin \xi L}{\xi L}\right) \int_{-\infty}^{\infty} d y e^{-i y q^{-}} \\
& -2 \frac{1-\cos \xi L}{\xi L} \int_{0}^{\infty} d y \sin \left(q^{-} y\right) \\
\approx & \left(1-\frac{\sin \xi L}{\xi L}\right) 2 \pi \delta\left(q^{-}\right) \\
& -2 \frac{1-\cos \xi L}{\xi L} \int_{0}^{\infty} d y \sin \left(q^{-} y\right) .
\end{aligned}
$$


Similarly as in previous section, for highly energetic jets

$$
\begin{aligned}
& \left(p+p_{1}\right)^{\rho} D_{\rho \sigma}^{>}(q)\left(p+p_{1}\right)^{\sigma} \\
& \quad \approx \theta\left(1-\frac{q_{0}^{2}}{\overrightarrow{\mathbf{q}}^{2}}\right) f\left(q_{0}\right) E^{+2} \frac{\boldsymbol{q}^{2}}{\overrightarrow{\mathbf{q}}^{2}} 2 \operatorname{Im}\left[\frac{1}{q^{2}-\Pi_{L}(q)}-\frac{1}{q^{2}-\Pi_{T}(q)}\right] .
\end{aligned}
$$

Finally, by using Eqs. (D22) and (D23), Eq. (D21) becomes

$$
\begin{aligned}
M_{1,0,2, R}+M_{1,0,2, L}= & -4 L g^{4} t_{c} t_{a} t_{a} t_{c} \int \frac{d^{3} p}{(2 \pi)^{3} 2 E}|J(p)|^{2} \\
& \times \int \frac{d^{3} k}{(2 \pi)^{3} 2 \omega} \frac{\boldsymbol{k}^{2}}{\left(\boldsymbol{k}^{2}+\chi\right)^{2}}\left(1-\frac{\sin \xi L}{\xi L}\right) \\
& \times I_{q}+8 L g^{4} t_{c} t_{a} t_{a} t_{c} \int \frac{d^{3} p}{(2 \pi)^{3} 2 E}|J(p)|^{2} \\
& \times \int \frac{d^{3} k}{(2 \pi)^{3} 2 \omega} \frac{\boldsymbol{k}^{2}}{\left(\boldsymbol{k}^{2}+\chi\right)^{2}} \frac{1-\cos \xi L}{\xi L} J_{q},
\end{aligned}
$$

where $I_{q}$ is given by Eq. (C15), and $J_{q}$ is given by

$$
\begin{aligned}
J_{q}= & \int_{0}^{\infty} d z \int \frac{d q_{0} d q_{z} d^{2} q}{(2 \pi)^{4}}\left[\sin \left(q_{0} z\right) \cos \left(q_{z} z\right)\right. \\
& \left.-\cos \left(q_{0} z\right) \sin \left(q_{z} z\right)\right] F\left(q_{0}, q_{z}, \boldsymbol{q}\right),
\end{aligned}
$$

In the above equation, we defined $F\left(q_{0}, q_{z}, \boldsymbol{q}\right)$ as

$$
\begin{aligned}
F\left(q_{0}, q_{z}, \boldsymbol{q}\right) \equiv & \frac{\boldsymbol{q}^{2}}{\overrightarrow{\mathbf{q}}^{2}} \frac{T}{q_{0}}\left\{\frac{2 \operatorname{Im} \Pi_{L}(q)}{\left[q^{2}-\operatorname{Re} \Pi_{L}(q)\right]^{2}+\operatorname{Im} \Pi_{L}(q)^{2}}\right. \\
& \left.-\frac{2 \operatorname{Im} \Pi_{T}(q)}{\left[q^{2}-\operatorname{Re} \Pi_{T}(q)\right]^{2}+\operatorname{Im} \Pi_{T}(q)^{2}}\right\} .
\end{aligned}
$$

$F\left(q_{0}, q_{z}, \boldsymbol{q}\right)$ is even function of both $q_{0}$ and $q_{z}$, leading to

$$
\begin{aligned}
& \int_{-\infty}^{\infty} d q_{0} \sin \left(q_{0} z\right) F\left(q_{0}, q_{z}, \boldsymbol{q}\right)=0 \\
& \int_{-\infty}^{\infty} d q_{z} \sin \left(q_{z} z\right) F\left(q_{0}, q_{z}, \boldsymbol{q}\right)=0,
\end{aligned}
$$

which consequently leads to

$$
J_{q}=0 .
$$

Finally, by using Eqs. (C15) and (D28), Eq. (D24) becomes

$$
\begin{aligned}
M_{1,0,2, R}+M_{1,0,2, L}= & -4 L T g^{4} t_{c} t_{a} t_{a} t_{c} \int \frac{d^{3} p}{(2 \pi)^{3} 2 E}|J(p)|^{2} \\
& \times \int \frac{d^{3} k}{(2 \pi)^{3} 2 \omega} \frac{d^{2} q}{(2 \pi)^{2}} \frac{\mu^{2}}{\boldsymbol{q}^{2}\left(\boldsymbol{q}^{2}+\mu^{2}\right)} \\
& \times \frac{\boldsymbol{k}^{2}}{\left(\boldsymbol{k}^{2}+\chi\right)^{2}}\left[1-\frac{\sin (\xi L)}{\xi L}\right] .
\end{aligned}
$$

\section{APPENDIX E: COMPUTATION OF DIAGRAMS $M_{1,0,3, C}$ AND $M_{1,0,4, C}$}

We will now calculate cut diagrams $M_{1,0,3, C}$ and $M_{1,0,4, C}$, shown in Fig. 4. We start with $M_{1,0,3, C}$ :

$$
\begin{aligned}
M_{1,0,3, C}= & \int \prod_{i=0}^{5} d^{4} x_{i} J\left(x_{0}\right) \Delta_{++}^{+}\left(x_{1}-x_{0}\right) v_{\mu}^{+}\left(x_{1}\right) \\
& \times D_{-+}^{\mu \nu}\left(x_{3}-x_{1}\right) \Delta_{++}^{+}\left(x_{2}-x_{1}\right) v_{\rho}^{+}\left(x_{2}\right) \\
& \times D_{-+}^{\rho \sigma}\left(x_{4}-x_{2}\right) \Delta_{-+}\left(x_{3}-x_{2}\right) v_{v}^{-}\left(x_{3}\right) \\
& \times \Delta_{--}^{-}\left(x_{4}-x_{3}\right) v_{\sigma}^{-}\left(x_{4}\right) \Delta_{--}^{-}\left(x_{5}-x_{4}\right) J\left(x_{5}\right) \\
& \times \theta\left(x_{1}^{+}-x_{0}^{+}\right) \theta\left(x_{2}^{+}-x_{1}^{+}\right) \theta\left[2 L-\left(x_{1}-x_{0}\right)^{+}\right] \\
& \times \theta\left(x_{4}^{+}-x_{5}^{+}\right) \theta\left(x_{3}^{+}-x_{4}^{+}\right) \theta\left[2 L-\left(x_{3}-x_{5}\right)^{+}\right] \\
= & -\int_{-\infty}^{\infty} \int_{0}^{\infty} \prod_{i=1}^{4} \frac{d p_{i}^{+} d^{2} p_{i}}{(2 \pi)^{3} 2 p_{i}^{+}} \int \frac{d^{3} k}{(2 \pi)^{3} 2 \omega} \\
& \times \int \frac{d^{3} p}{(2 \pi)^{3} 2 E} \int \frac{d^{4} q}{(2 \pi)^{4}} g^{4} t_{a} t_{c} t_{a} t_{c} \\
& \times\left(p_{1}+p_{2}\right)^{\mu} D_{\mu \nu}^{>}(q)\left(p+p_{3}\right)^{\nu} \\
& \times\left(p+p_{1}\right)^{\rho} P_{\rho \sigma}(k)\left(p_{3}+p_{4}\right)^{\sigma} I,
\end{aligned}
$$

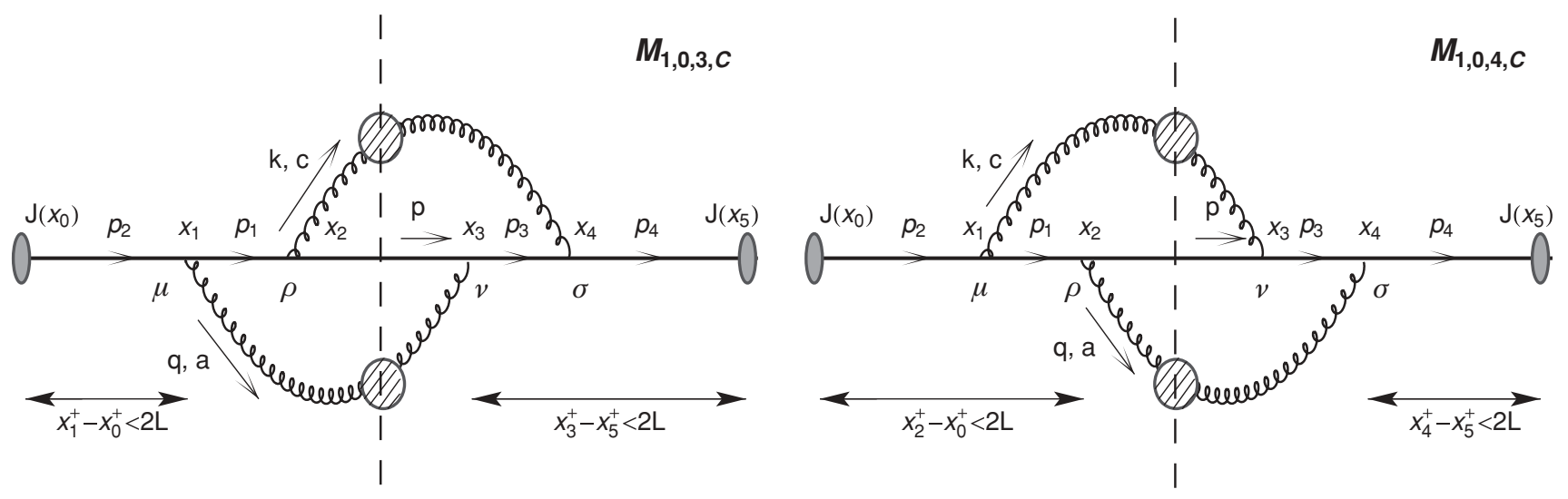

FIG. 4. Feynman diagrams $M_{1,0,3, C}$ and $M_{1,0,4, C}$ contributing to the radiative energy loss to first order in opacity, labeled the same way as in Fig. 1. 
where

$$
\begin{aligned}
I= & \int \prod_{i=0}^{5} d^{4} x_{i} \theta\left(x_{1}^{+}-x_{0}^{+}\right) \theta\left(x_{2}^{+}-x_{1}^{+}\right) \theta\left[2 L-\left(x_{1}-x_{0}\right)^{+}\right] \\
& \times \theta\left(x_{4}^{+}-x_{5}^{+}\right) \theta\left(x_{3}^{+}-x_{4}^{+}\right) \theta\left[2 L-\left(x_{3}-x_{5}\right)^{+}\right] \\
& \times e^{-i p_{2} \cdot\left(x_{1}-x_{0}\right)} e^{-i q \cdot\left(x_{3}-x_{1}\right)} e^{-i p_{1} \cdot\left(x_{2}-x_{1}\right)} e^{-i k \cdot\left(x_{4}-x_{2}\right)} \\
& \times e^{-i p \cdot\left(x_{3}-x_{2}\right)} e^{-i p_{3} \cdot\left(x_{4}-x_{3}\right)} e^{-i p_{4} \cdot\left(x_{5}-x_{4}\right)} J\left(x_{0}\right) J\left(x_{5}\right) \\
= & |J(p)|^{2}(2 \pi)^{3} \delta\left[\left(p_{2}-p-k-q\right)^{+}\right] \delta^{2}\left(\boldsymbol{p}_{2}-\boldsymbol{p}-\boldsymbol{k}-\boldsymbol{q}\right) \\
& \times(2 \pi)^{3} \delta\left[\left(p_{1}-p-k\right)^{+}\right] \delta^{2}\left(\boldsymbol{p}_{1}-\boldsymbol{p}-\boldsymbol{k}\right) \\
& \times(2 \pi)^{3} \delta\left[\left(p_{3}-p-q\right)^{+}\right] \delta^{2}\left(\boldsymbol{p}_{3}-\boldsymbol{p}-\boldsymbol{q}\right) \\
& \times(2 \pi)^{3} \delta\left[\left(p_{4}-p_{3}-k\right)^{+}\right] \delta^{2}\left(\boldsymbol{p}_{4}-\boldsymbol{p}_{3}-\boldsymbol{k}\right) I_{1}
\end{aligned}
$$

and where

$$
\begin{aligned}
I_{1}= & \int_{0}^{\infty} d x_{2}^{\prime+} e^{-\frac{i}{2}\left(p_{1}-p-k\right)^{-} x_{2}^{\prime+}} \int_{0}^{2 L} d x_{1}^{\prime+} e^{-\frac{i}{2}\left(p_{2}-p-k-q\right)^{-} x_{1}^{\prime+}} \\
& \times \int_{0}^{2 L} d x_{3}^{\prime+} e^{\frac{i}{2}\left(p_{3}-p-q\right)^{-} x_{3}^{\prime+}} \int_{0}^{x_{3}} d x_{4}^{\prime+} e^{\frac{i}{2}\left(p_{4}-p_{3}-k\right)^{-} x_{4}^{\prime+}} \\
= & \frac{-16}{\left(p_{1}-p-k\right)^{-}\left(p_{4}-p_{3}-k\right)^{-}} \frac{e^{-i\left(p_{2}-p-k-q\right) L}-1}{\left(p_{2}-p-k-q\right)^{-}} \\
& \times\left[\frac{e^{i\left(p_{4}-p-k-q\right)^{-} L}-1}{\left(p_{4}-p-k-q\right)^{-}}-\frac{e^{i\left(p_{3}-p-q\right)^{-} L}-1}{\left(p_{3}-p-q\right)^{-}}\right]
\end{aligned}
$$

Similarly as in previous sections, by using $\delta$ functions from Eqs. (E2) and (C5), we obtain $p_{4}^{-}=p_{2}^{-}$and

$$
\left(p_{1}-p-k\right)^{-} \approx\left(p_{4}-p_{3}-k\right)^{-}=-\xi .
$$

$I_{1}$ then becomes

$$
\begin{aligned}
I_{1}- & -\frac{16}{\xi^{2}} \frac{4 \sin ^{2}\left[\left(p_{2}-p-k-q\right)^{-} \frac{L}{2}\right]}{\left[\left(p_{2}-p-k-q\right)^{-}\right]^{2}} \\
& \times\left[1-\frac{\left(p_{2}-p-k-q\right)^{-}}{e^{-i\left(p_{2}-p-k-q\right)^{-} L}-1} \frac{e^{i\left(p_{3}-p-q\right)^{-} L}-1}{\left(p_{3}-p-q\right)^{-}}\right] \\
\approx & -\frac{16}{\xi^{2}} 2 \pi L \delta\left[\left(p_{2}-p-k-q\right)^{-}\right]\left[1-\lim _{\left(p_{2}-p-k-q\right)^{-} \rightarrow 0}\right. \\
& \left.\times \frac{\left(p_{2}-p-k-q\right)^{-}}{e^{-i\left(p_{2}-p-k-q\right)^{-} L}-1} \frac{e^{i\left\{\left(p_{2}-p-k-q\right)^{-}+\xi\right\} L}-1}{\left(p_{2}-p-k-q\right)^{-}+\xi}\right] \\
\approx & -\frac{16}{\xi^{2}} 2 \pi L \delta\left(q_{0}-q_{z}\right)\left[1-\frac{\sin (\xi L)}{\xi L}+i \frac{\cos (\xi L)-1}{\xi L}\right],
\end{aligned}
$$

where similarly to previous sections, in the second step we used Eq. (A9) and the fact that finite-size effects are negligible for collisional interactions. That is, we used $\frac{4 \sin ^{2}\left[\left(p_{2}-p-k-q\right)^{-} \frac{L}{2}\right]}{\left[\left(p_{2}-p-k-q\right)^{-}\right]^{2}} \approx$ $2 \pi L \delta\left(q^{-}-\xi\right) \approx 2 \pi L \delta\left(q_{0}-q_{z}\right)$.

Finally, by using Eqs. (E2) and (E5), Eq. (E1) becomes

$$
\begin{aligned}
M_{1,0,3, C}= & -4 L \operatorname{Tg}^{4} t_{a} t_{c} t_{a} t_{c} \int \frac{d^{3} p}{(2 \pi)^{3} 2 E}|J(p)|^{2} \\
& \times \int \frac{d^{3} k}{(2 \pi)^{3} 2 \omega} \frac{\boldsymbol{k}^{2}}{\left(\boldsymbol{k}^{2}+\chi\right)^{2}} \int \frac{d^{2} q}{(2 \pi)^{2}} \frac{\mu^{2}}{\boldsymbol{q}^{2}\left(\boldsymbol{q}^{2}+\mu^{2}\right)} \\
& \times\left[1-\frac{\sin (\xi L)}{\xi L}-i \frac{1-\cos (\xi L)}{\xi L}\right] .
\end{aligned}
$$

Because $M_{1,0,4, C}=\left(M_{1,0,4, C}\right)^{*}$, we obtain

$$
\begin{aligned}
M_{1,0,3, C}+M_{1,0,4, C}= & -8 L \operatorname{Tg}^{4} t_{a} t_{c} t_{a} t_{c} \int \frac{d^{3} p}{(2 \pi)^{3} 2 E}|J(p)|^{2} \\
& \times \int \frac{d^{3} k}{(2 \pi)^{3} 2 \omega} \frac{d^{2} q}{(2 \pi)^{2}} \frac{\mu^{2}}{\boldsymbol{q}^{2}\left(\boldsymbol{q}^{2}+\mu^{2}\right)} \\
& \times \frac{\boldsymbol{k}^{2}}{\left(\boldsymbol{k}^{2}+\chi\right)^{2}}\left[1-\frac{\sin (\xi L)}{\xi L}\right] . \quad \text { (E7) }
\end{aligned}
$$

\section{APPENDIX F: COMPUTATION OF DIAGRAMS $M_{1,0,3, R}$ AND $M_{1,0,4, L}$}

We will now calculate the cut diagrams $M_{1,0,3, R}$ and $M_{1,0,4, L}$, shown in Fig. 5 . We start with $M_{1,0,3, R}$ :

$$
\begin{aligned}
M_{1,0,3, R}= & \int \prod_{i=0}^{5} d^{4} x_{i} J\left(x_{0}\right) \Delta_{++}^{+}\left(x_{1}-x_{0}\right) v_{\mu}^{+}\left(x_{1}\right) \\
& \times D_{++}^{+\mu \nu}\left(x_{3}-x_{1}\right) \Delta_{++}^{+}\left(x_{2}-x_{1}\right) v_{\rho}^{+}\left(x_{2}\right) \\
& \times D_{-+}^{\rho \sigma}\left(x_{4}-x_{2}\right) \Delta_{++}^{+}\left(x_{3}-x_{2}\right) v_{\lambda}^{+}\left(x_{3}\right) \\
& \times \Delta_{-+}\left(x_{4}-x_{3}\right) v_{\sigma}^{-}\left(x_{4}\right) \Delta_{--}^{-}\left(x_{5}-x_{4}\right) J\left(x_{5}\right) \\
& \times \prod_{i=0}^{3} \theta\left(x_{i+1}^{+}-x_{i}^{+}\right) \theta\left(x_{4}^{+}-x_{5}^{+}\right) \theta\left[2 L-\left(x_{1}-x_{0}\right)^{+}\right] \\
= & \int_{-\infty}^{\infty} \int_{0}^{\infty} \frac{d p_{i}^{+} d^{2} p_{i}}{(2 \pi)^{3} 2 p_{i}^{+}} \int \frac{d^{3} k}{(2 \pi)^{3} 2 \omega} \frac{d^{3} p}{(2 \pi)^{3} 2 E} \\
& \times \frac{d^{4} q}{(2 \pi)^{4}} g^{4} t_{a} t_{c} t_{a} t_{c}\left(p_{2}+p_{3}\right)^{\mu} D_{\mu \lambda}^{>}(q)\left(p+p_{1}\right)^{\lambda} \\
& \times\left(p_{1}+p_{2}\right)^{\rho} P_{\rho \sigma}(k)\left(p+p_{4}\right)^{\sigma} I,
\end{aligned}
$$

where

$$
\begin{aligned}
I= & \int \prod_{i=0}^{5} d^{4} x_{i} \prod_{j=0}^{3} \theta\left(x_{j+1}^{+}-x_{j}^{+}\right) \\
& \times \theta\left[2 L-\left(x_{1}-x_{0}\right)^{+}\right] \theta\left(x_{4}^{+}-x_{5}^{+}\right) J\left(x_{0}\right) J\left(x_{5}\right) \\
& \times e^{-i p_{3} \cdot\left(x_{1}-x_{0}\right)} e^{-i q \cdot\left(x_{3}-x_{1}\right)} e^{-i p_{2} \cdot\left(x_{2}-x_{1}\right)} e^{-i p_{1} \cdot\left(x_{3}-x_{2}\right)} \\
& \times e^{-i p \cdot\left(x_{4}-x_{3}\right)} e^{-i k \cdot\left(x_{4}-x_{1}\right)} e^{-i p_{4} \cdot\left(x_{5}-x_{4}\right)} \\
= & |J(p)|^{2}(2 \pi)^{3} \delta\left[\left(p_{3}-p-k\right)^{+}\right] \delta^{2}\left(\boldsymbol{p}_{3}-\boldsymbol{p}-\boldsymbol{k}\right)(2 \pi)^{3} \\
& \times \delta\left[\left(p_{2}-p_{1}-k\right)^{+}\right] \delta^{2}\left(\boldsymbol{p}_{2}-\boldsymbol{p}_{1}-\boldsymbol{k}\right) \\
& \times(2 \pi)^{3} \delta\left[\left(p_{1}+q-p\right)^{+}\right] \delta^{2}\left(\boldsymbol{p}_{1}+\boldsymbol{q}-\boldsymbol{p}\right)(2 \pi)^{3} \\
& \times \delta\left[\left(p_{4}-p-k\right)^{+}\right] \delta^{2}\left(\boldsymbol{p}_{4}-\boldsymbol{p}-\boldsymbol{k}\right) I_{1} .
\end{aligned}
$$

Here

$$
\begin{aligned}
I_{1}= & \int_{0}^{\infty} d x_{3}^{\prime+} e^{-\frac{i}{2}\left(p_{1}+q-p\right)^{-} x_{3}^{+}} \int_{0}^{x_{3}^{+}} d x_{2}^{\prime+} e^{-\frac{i}{2}\left(p_{2}-p_{1}-k\right)^{-} x_{2}^{\prime+}} \\
& \times \int_{0}^{2 L} d x_{1}^{\prime+} e^{\frac{-i}{2}\left(p_{3}-p-k\right)^{-} x_{1}^{\prime+}} \int_{0}^{\infty} d x_{4}^{\prime+} e^{\frac{i}{2}\left(p_{4}-p-k\right)^{-} x_{4}^{\prime+}},
\end{aligned}
$$

where we defined $x_{1}^{\prime}=x_{1}-x_{0}, x_{2}^{\prime}=x_{2}-x_{1}, x_{3}^{\prime}=x_{3}-x_{1}$, and $x_{4}^{\prime}=x_{4}-x_{5}$. By using $\delta$ functions from Eqs. (F2) 


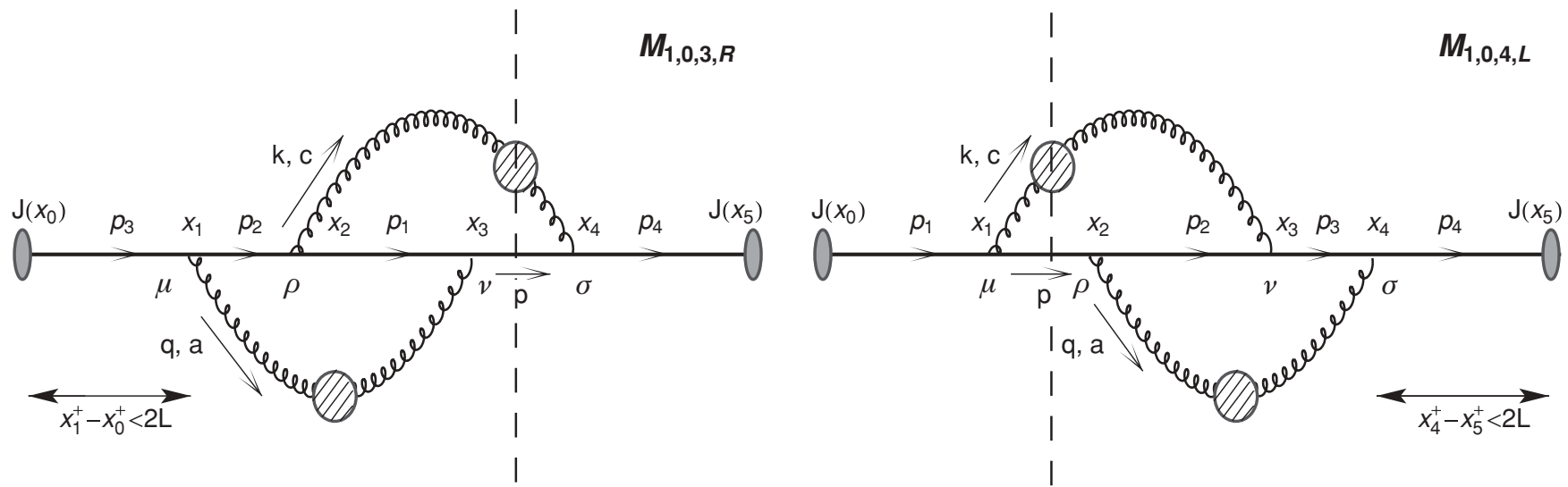

FIG. 5. Feynman diagrams $M_{1,0,3, R}$ and $M_{1,0,4, L}$ contributing to the radiative energy loss to first order in opacity, labeled the same way as in Fig. 1.

and (C5), we obtain in soft-gluon, soft-rescattering approximation:

$$
\begin{gathered}
\left(p_{3}-p-k\right)^{-}=\left(p_{4}-p-k\right)^{-} \approx\left(p_{2}-k-p_{1}\right)^{-} \approx-\xi \\
\left(p_{1}-p\right)^{-} \approx \frac{\boldsymbol{q}^{2}+2 \boldsymbol{k} \boldsymbol{q}}{p^{+}} \ll \xi \ll|\boldsymbol{q}|,|\boldsymbol{k}|, q_{z}, \\
\text { leading to }\left(p_{1}-p\right)^{-}+q^{-} \approx q^{-} .
\end{gathered}
$$

$I_{1}$ then becomes

$$
\begin{aligned}
I_{1}= & \frac{-8}{\xi^{3}}[\sin \xi L+i(1-\cos \xi L)] \\
& \times \int_{0}^{\infty} d x_{3}^{\prime+}\left(e^{-\frac{i}{2}\left(q^{-}-\xi\right) x_{3}^{\prime+}}-e^{-\frac{i}{2} q^{-} x_{3}^{\prime+}}\right) .
\end{aligned}
$$

Finally, by using Eqs. (F2) and (F6) Eq. (F1) becomes

$$
\begin{aligned}
M_{1,0,3, R}= & 2 L g^{4} t_{a} t_{c} t_{a} t_{c} \int \frac{d^{3} p}{(2 \pi)^{3} 2 E}|J(p)|^{2} \\
& \times \int \frac{d^{3} k}{(2 \pi)^{3} 2 \omega} \frac{\boldsymbol{k}^{2}}{\left(\boldsymbol{k}^{2}+\chi\right)^{2}} \int \frac{d^{4} q}{(2 \pi)^{4}} f\left(q_{0}\right) \\
& \times \frac{\boldsymbol{q}^{2}}{\overrightarrow{\mathbf{q}}^{2}} 2 \operatorname{Im}\left(\frac{1}{q^{2}-\Pi_{L}}-\frac{1}{q^{2}-\Pi_{T}}\right) \\
& \times\left(\frac{\sin \xi L}{\xi L}+i \frac{1-\cos \xi L}{\xi L}\right) \\
& \times \int_{0}^{\infty} d x_{3}^{\prime+}\left[e^{-\frac{i}{2}\left(q^{-}-\xi\right) x_{3}^{\prime+}}-e^{-\frac{i}{2} q^{-} x_{3}^{\prime+}}\right] .
\end{aligned}
$$

Note that $M_{1,0,4, L}=\left(M_{1,0,3, R}\right)^{*}$, leading to

$$
\begin{aligned}
& M_{1,0,3, R}+M_{1,0,4, L} \\
&=4 L g^{4} t_{a} t_{c} t_{c} t_{a} \int \frac{d^{3} p}{(2 \pi)^{3} 2 E}|J(p)|^{2} \int \frac{d^{3} k}{(2 \pi)^{3} 2 \omega} \frac{\boldsymbol{k}^{2}}{\left(\boldsymbol{k}^{2}+\chi\right)^{2}} \\
& \quad \times \int \frac{d^{4} q}{(2 \pi)^{4}} f\left(q_{0}\right) \frac{\boldsymbol{q}^{2}}{\overrightarrow{\mathbf{q}}^{2}} 2 \operatorname{Im}\left(\frac{1}{q^{2}-\Pi_{L}}-\frac{1}{q^{2}-\Pi_{T}}\right) \theta \\
& \quad \times\left(1-\frac{q_{0}^{2}}{\overrightarrow{\mathbf{q}}^{2}}\right)\left\{\frac{\sin \xi L}{\xi L}\left[\delta\left(q^{-}-\xi\right)-\delta\left(q^{-}\right)\right]\right. \\
&+\left.2 \frac{1-\cos \xi L}{\xi L} \int_{0}^{\infty} d y\left[\sin \left(q^{-}-\xi\right) y-\sin \left(q^{-} y\right)\right]\right\},
\end{aligned}
$$

where $y \equiv \frac{x_{3}^{\prime}}{2}$. Let us define

$$
\begin{aligned}
F\left(q_{0}, q_{z}^{2}, \boldsymbol{q}^{2}\right)= & f\left(q_{0}\right) \frac{\boldsymbol{q}^{2}}{\overrightarrow{\mathbf{q}}^{2}} 2 \operatorname{Im}\left[\frac{1}{q^{2}-\Pi_{L}(q)}\right. \\
& \left.-\frac{1}{q^{2}-\Pi_{T}(q)}\right] \theta\left(1-\frac{q_{0}^{2}}{\overrightarrow{\mathbf{q}}^{2}}\right) .
\end{aligned}
$$

In the high-temperature limit $F\left(q_{0}, q_{z}^{2}, \boldsymbol{q}^{2}\right)$ is even function of $q_{0}$ and $q_{z}$. Then $\left.\frac{\partial F\left(q_{0}, q_{z}^{2}, \boldsymbol{q}^{2}\right)}{\partial q_{0}}\right|_{q_{0}=q_{z}}$ is an odd function of $q_{z}$, leading to

$$
\begin{gathered}
\int \frac{d^{4} q}{(2 \pi)^{4}} F\left(q_{0}, q_{z}^{2}, \boldsymbol{q}^{2}\right)\left[\delta\left(q_{0}-q_{z}-\xi\right)-\delta\left(q_{2}-q_{z}\right)\right] \\
=\left.\xi \int \frac{d^{3} q}{(2 \pi)^{3}} \frac{\partial F\left(q_{0}, q_{z}^{2}, \boldsymbol{q}^{2}\right)}{\partial q_{0}}\right|_{q_{0}=q_{z}}=0 .
\end{gathered}
$$

We will now compute the second part of the integral in Eq. (F8):

$$
\begin{aligned}
& \int \frac{d^{4} q}{(2 \pi)^{4}} F\left(q_{0}, q_{z}^{2}, \boldsymbol{q}^{2}\right) \int_{0}^{\infty} d y\left[\sin \left(q_{0}-q_{z}-\xi\right) y\right. \\
& \left.\quad-\sin \left(q_{0}-q_{z}\right) y\right] .
\end{aligned}
$$

To do this we first concentrate on

$$
\begin{aligned}
\int_{0}^{\infty} & d y \int_{0}^{\infty} \frac{d^{4} q}{(2 \pi)^{4}} F\left(q_{0}, q_{z}^{2}, \boldsymbol{q}^{2}\right) \sin \left[\left(q_{0}-q_{z}-\xi\right) y\right] \\
= & \int_{0}^{\infty} d y \int_{0}^{\infty} \frac{d^{4} q}{(2 \pi)^{4}} F\left(q_{0}, q_{z}^{2}, \boldsymbol{q}^{2}\right) \\
& \times\left\{\sin \left(q_{0} y\right) \cos \left[\left(q_{z}-\xi\right) y\right]-\cos \left(q_{0} y\right) \sin \left[\left(q_{z}-\xi\right) y\right]\right\} \\
= & \int_{0}^{\infty} d y \int_{0}^{\infty} \frac{d^{3} q}{(2 \pi)^{4}} \cos \left[\left(q_{z}-\xi\right) y\right] \\
& \times \int d q_{0} F\left(q_{0}, q_{z}^{2}, \boldsymbol{q}^{2}\right) \sin \left(q_{0} y\right)-\int_{0}^{\infty} d y \\
& \times \int \frac{d q_{0} d^{2} q}{(2 \pi)^{4}} \cos \left(q_{0} y\right) \int d q_{z} g\left(q_{0}, q_{z}^{2}, \boldsymbol{q}^{2}\right) \\
& \times \sin \left[\left(q_{z}-\xi\right) y\right] .
\end{aligned}
$$


Because $\xi \ll q_{z},|\boldsymbol{q}|$ we can assume $q_{z}-\xi \approx q_{z}$. Therefore $\sin \left[\left(q_{z}-\xi\right) y\right] \approx \sin q_{z} y$, leading to $\int d q_{z} F\left(q_{0}, q_{z}^{2}, \boldsymbol{q}^{2}\right)$ $\sin \left[\left(q_{z}-\xi\right) y\right] \approx \int d q_{z} F\left(q_{0}, q_{z}^{2}, \boldsymbol{q}^{2}\right) \sin \left(q_{z} y\right)=0$. Similarly, for the second part of the integral in Eq. (F10) we also obtain 0 , which finally leads to

$$
M_{1,0,3, R}+M_{1,0,4, L} \approx 0 .
$$

\section{APPENDIX G: COMPUTATION OF DIAGRAMS $M_{1,0,5, R}$ AND $M_{1,0,6, L}$}

We will now calculate the cut diagrams $M_{1,0,5, R}$ and $M_{1,0,6, L}$, shown in Fig. 6. We start with $M_{1,0,5, R}$ :

$$
\begin{aligned}
M_{1,0,5, R}= & \int \prod_{i=0}^{5} d^{4} x_{i} J\left(x_{0}\right) \Delta_{++}^{+}\left(x_{1}-x_{0}\right) v_{\mu}^{+}\left(x_{1}\right) \\
& \times D_{++}^{+\mu v}\left(x_{2}-x_{1}\right) \Delta_{++}^{+}\left(x_{2}-x_{1}\right) v_{v}^{+}\left(x_{2}\right) \\
& \times \Delta_{++}^{+}\left(x_{3}-x_{2}\right) v_{\rho}^{+}\left(x_{3}\right) D_{-+}^{\rho \sigma}\left(x_{4}-x_{3}\right) \\
& \times \Delta_{-+}\left(x_{4}-x_{3}\right) v_{\sigma}^{-}\left(x_{4}\right) \Delta_{--}^{-}\left(x_{5}-x_{4}\right) J\left(x_{5}\right) \\
& \times \theta\left(x_{1}^{+}-x_{0}^{+}\right) \theta\left(x_{2}^{+}-x_{1}^{+}\right) \\
& \times \theta\left(x_{3}^{+}-x_{2}^{+}\right) \theta\left(x_{5}^{+}-x_{4}^{+}\right) \theta\left[2 L-\left(x_{1}-x_{0}\right)^{+}\right] \\
= & g^{4} t_{a} t_{a} t_{c} t_{c} \int_{0}^{\infty} \int_{-\infty}^{\infty} \prod_{i=1}^{4} \frac{d p_{i}^{+} d^{2} p_{i}}{(2 \pi)^{3} 2 p_{i}^{+}} \\
& \times \int \frac{d^{3} k}{(2 \pi)^{3} 2 \omega} \frac{d^{3} p}{(2 \pi)^{3} 2 E} \int \frac{d^{4} q}{(2 \pi)^{4}} \\
& \times\left\{\left(p_{2}+p_{3}\right)^{\mu} D_{\mu \nu}^{>}(q)\left(p_{1}+p_{2}\right)^{\nu}\right\} \\
& \times\left\{\left(p+p_{1}\right)^{\rho} P_{\rho \sigma}(k)\left(p+p_{4}\right)^{\sigma}\right\} I, \quad(\mathrm{G} 1)
\end{aligned}
$$

where

$$
\begin{aligned}
I= & \int \prod_{i=0}^{5} d^{4} x_{i} \theta\left(x_{1}^{+}-x_{0}^{+}\right) \theta\left(x_{2}^{+}-x_{1}^{+}\right) \theta\left(x_{3}^{+}-x_{2}^{+}\right) \\
& \times \theta\left(x_{4}^{+}-x_{5}^{+}\right) \theta\left[2 L-\left(x_{1}^{+}-x_{0}^{+}\right)\right] \\
& \times e^{-i p_{3} \cdot\left(x_{1}-x_{0}\right)} e^{-i q \cdot\left(x_{2}-x_{1}\right)} e^{-i p_{2} \cdot\left(x_{2}-x_{1}\right)} e^{-i p_{1} \cdot\left(x_{3}-x_{2}\right)} \\
& \times e^{-i k\left(x_{4}-x_{3}\right)} e^{-i p \cdot\left(x_{4}-x_{3}\right)} e^{-i p_{4} \cdot\left(x_{5}-x_{4}\right)} J\left(x_{0}\right) J\left(x_{5}\right)
\end{aligned}
$$

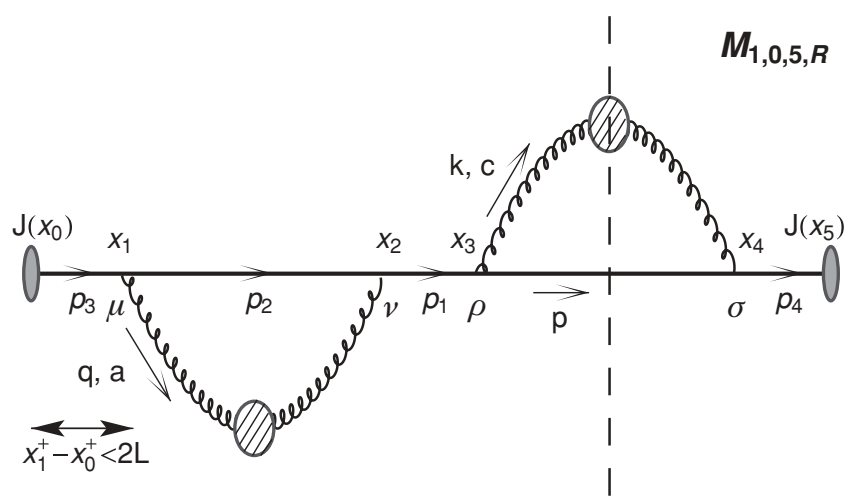

$$
\begin{aligned}
= & |J(p)|^{2}(2 \pi)^{3} \delta\left[\left(p_{3}-p-k\right)^{+}\right] \delta^{2}\left(\boldsymbol{p}_{3}-\boldsymbol{p}-\boldsymbol{k}\right) \\
& \times(2 \pi)^{3} \delta\left[\left(p_{2}+q-p-k\right)^{+}\right] \delta^{2}\left(\boldsymbol{p}_{2}+\boldsymbol{q}-\boldsymbol{p}-\boldsymbol{k}\right) \\
& \times(2 \pi)^{3} \delta\left[\left(p_{1}-p-k\right)^{+}\right] \delta^{2}\left(\boldsymbol{p}_{1}-\boldsymbol{p}-\boldsymbol{k}\right)(2 \pi)^{3} \\
& \times \delta\left[\left(p_{4}-p-k\right)^{+}\right] \delta^{2}\left(\boldsymbol{p}_{4}-\boldsymbol{p}-\boldsymbol{k}\right) I_{1}
\end{aligned}
$$

and where

$$
\begin{aligned}
I_{1}= & \int_{0}^{2 L} d x_{1}^{\prime+} e^{-\frac{i}{2}\left(p_{3}-p-k\right)^{-} x_{1}^{++}} \int_{0}^{\infty} d x_{4}^{\prime+} e^{\frac{i}{2}\left(p_{4}-p-k\right)^{-} x_{4}^{\prime+}} \\
& \times \int_{0}^{\infty} d x_{3}^{\prime+} e^{-\frac{i}{2}\left(p_{1}-p-k\right)^{-} x_{3}^{\prime+}} \int_{0}^{\infty} d x_{2}^{\prime+} e^{-\frac{i}{2}\left(p_{2}+q-p-k\right)^{-} x_{2}^{\prime+}} .
\end{aligned}
$$

Here we defined $x_{1}^{\prime}=x_{1}-x_{0}, x_{2}^{\prime}=x_{2}-x_{1}, x_{3}^{\prime}=x_{3}-x_{2}$, $x_{4}^{\prime}=x_{4}-x_{5}$.

By using $\delta$ functions from Eqs. (G2) and (C5) we obtain $p_{1}^{-}=p_{3}^{-}=p_{4}^{-}$and

$$
\left(p_{3}-p-k\right)^{-}=\left(p_{1}-p-k\right)^{-}=\left(p_{4}-p-k\right)^{-}=-\xi .
$$

Equation (G3) then becomes

$$
\begin{aligned}
I_{1} & =\frac{16 L}{\xi^{2}}\left(\frac{e^{-i \xi L}-1}{-i \xi L}\right) \int_{0}^{\infty} d y e^{-i y\left(p_{2}-p-k+q\right)^{-}} \\
& \approx \frac{16 L}{\xi^{2}}\left(\frac{\sin \xi L}{\xi L}-i \frac{\cos \xi L-1}{\xi L}\right) \int_{0}^{\infty} d y e^{-i y q^{-}},
\end{aligned}
$$

where $y=x_{2}^{\prime} / 2$, and in the last step we used $\left(p_{2}-p-k+\right.$ $q)^{-}=q^{-}-\xi \approx q^{-}$for small $\xi$.

Finally, by using Eqs. (G2) and (G5), Eq. (G1) becomes

$$
\begin{aligned}
M_{1,0,5, R}= & -4 L T g^{4} t_{a} t_{a} t_{c} t_{c} \int \frac{d^{3} p}{(2 \pi)^{3} 2 E}|J(p)|^{2} \int \frac{d^{3} k}{(2 \pi)^{3} 2 \omega} \\
& \times \frac{\boldsymbol{k}^{2}}{\left(\boldsymbol{k}^{2}+\chi\right)^{2}} \int \frac{d^{4} q}{(2 \pi)^{4}} \theta\left(1-\frac{q_{0}^{2}}{\vec{q}^{2}}\right) \frac{1}{q_{0}} \frac{\boldsymbol{q}^{2}}{\vec{q}^{2}} \\
& \times 2 \operatorname{Im}\left[\frac{1}{q^{2}-\Pi_{L}(q)}-\frac{1}{q^{2}-\Pi_{T}(q)}\right] \\
& \times\left(\frac{\sin \xi L}{\xi L}-i \frac{\cos \xi L-1}{\xi L}\right) \int_{0}^{\infty} d y e^{-i y q^{-}} .
\end{aligned}
$$

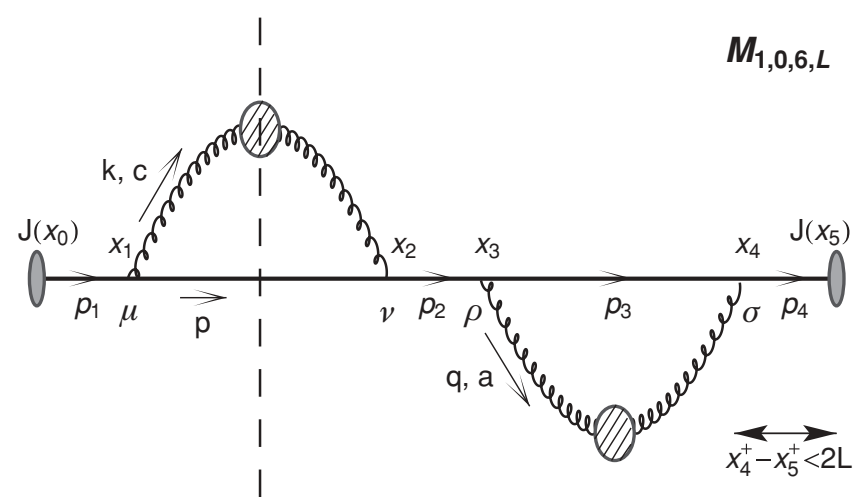

FIG. 6. Feynman diagrams $M_{1,0,5, R}$ and $M_{1,0,6, L}$ contributing to the radiative energy loss to first order in opacity, labeled the same way as in Fig. 1. 
Because $M_{1,0,6, L}=\left(M_{1,0,5, R}\right)^{*}$, it is straightforward to obtain [note that, similarly to Appendix D, $\left(\frac{\cos \xi L-1}{\xi L}\right)$ part will vanish under integration over $q$ ]:

$$
\begin{aligned}
M_{1,0,5, R}+M_{1,0,6, L}= & -4 L T g^{4} t_{a} t_{a} t_{c} t_{c} \int \frac{d^{3} p}{(2 \pi)^{3} 2 E}|J(p)|^{2} \\
& \times \int \frac{d^{3} k}{(2 \pi)^{3} 2 \omega} \frac{d^{2} q}{(2 \pi)^{2}} \frac{\mu^{2}}{q^{2}\left(q^{2}+\mu^{2}\right)} \\
& \times \frac{\boldsymbol{k}^{2}}{\left(\boldsymbol{k}^{2}+\chi\right)^{2}} \frac{\sin \xi L}{\xi L} .
\end{aligned}
$$

\section{APPENDIX H: COMPUTATION OF DIAGRAMS

$$
M_{1,1,1, C} \text { AND } M_{1,1,2, C}
$$

In Appendices $\mathrm{H}-\mathrm{K}$ we present in some detail the calculation of the diagrams where only one end of the exchanged gluon $q$ is attached to the heavy quark, i.e., one end is attached to the radiated gluon $k$ and consequently one three-gluon vertex is involved in the process. In this appendix, we start with the calculation of the diagrams shown in Fig. 7.

We will first calculate the cut diagram $M_{1,1,1, C}$ :

$$
\begin{aligned}
M_{1,1,1, C}= & \int \prod_{i=0}^{5} d x_{i} J\left(x_{0}\right) \Delta_{++}^{+}\left(x_{1}-x_{0}\right) v_{\mu}^{+}\left(x_{1}\right) \\
& \times D_{++}^{+\mu \rho_{1}}\left(x_{2}-x_{1}\right) v_{\rho_{1} \rho_{2} \rho_{3}}^{+}\left(x_{2}\right) \Delta_{-+}\left(x_{3}-x_{1}\right) \\
& \times D_{-+}^{\rho_{3} v}\left(x_{3}-x_{2}\right) v_{v}^{-}\left(x_{3}\right) D_{-+}^{\rho_{2} \sigma}\left(x_{4}-x_{2}\right) v_{\sigma}^{-}\left(x_{4}\right) \\
& \times \Delta_{--}^{-}\left(x_{4}-x_{3}\right) \Delta_{--}^{-}\left(x_{5}-x_{4}\right) J\left(x_{5}\right) \\
& \times \theta\left(x_{1}^{+}-x_{0}^{+}\right) \theta\left(x_{2}^{+}-x_{1}^{+}\right) \theta\left[2 L-\left(x_{2}-x_{0}\right)^{+}\right] \\
& \times \theta\left(x_{3}^{+}-x_{4}^{+}\right) \theta\left(x_{4}^{+}-x_{5}^{+}\right) \theta\left[2 L-\left(x_{4}-x_{5}\right)^{+}\right] \\
= & -\int_{-\infty}^{\infty} \int_{0}^{\infty} \prod_{i=1}^{3} \frac{d p_{i}^{+} d^{2} p_{i}}{(2 \pi)^{3} 2 p_{i}^{+}} \frac{d k_{1}^{+} d^{2} k_{1}}{(2 \pi)^{3} 2 k_{1}^{+}} \\
& \times \int \frac{d^{3} k}{(2 \pi)^{3} 2 \omega} \frac{d^{3} p}{(2 \pi)^{3} 2 E} \frac{d^{4} q}{(2 \pi)^{4}}(-i) g^{4} f^{c b a} t_{c} t_{b} t_{a} \\
& \times\left(p+p_{1}\right)^{\mu} P_{\mu \rho_{1}}\left(k_{1}\right)\left[g^{\rho_{1} \rho_{3}}\left(k_{1}+k\right)^{\rho_{2}}\right. \\
& \left.+g^{\rho_{2} \rho_{3}}(q-k)^{\rho_{1}}+g^{\rho_{1} \rho_{2}}\left(-k_{1}-q\right)^{\rho_{3}}\right] \\
& \times P_{\rho_{3} v}(k) D_{\rho_{2} \sigma}^{>}(q)\left(p+p_{2}\right)^{v}\left(p_{2}+p_{3}\right)^{\sigma} I, \quad(\mathrm{H} 1)
\end{aligned}
$$

$$
M_{1,1,1, C}
$$

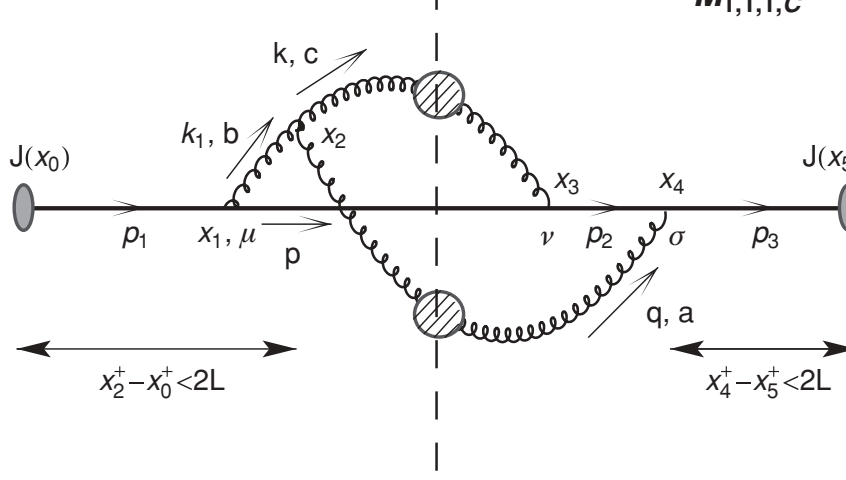

where

$$
\begin{aligned}
I= & \int \prod_{i=0}^{5} d x_{i} \theta\left(x_{1}^{+}-x_{0}^{+}\right) \theta\left(x_{2}^{+}-x_{1}^{+}\right) \theta\left[2 L-\left(x_{2}-x_{0}\right)^{+}\right] \\
& \times \theta\left(x_{3}^{+}-x_{4}^{+}\right) \theta\left(x_{4}^{+}-x_{5}^{+}\right) \theta\left[2 L-\left(x_{4}-x_{5}\right)^{+}\right] \\
& \times e^{-i p_{1}\left(x_{1}-x_{0}\right)} e^{-i k_{1}\left(x_{2}-x_{1}\right)} e^{-i p\left(x_{3}-x_{1}\right)} e^{-i k\left(x_{3}-x_{2}\right)} e^{-i q\left(x_{4}-x_{2}\right)} \\
& \times e^{-i p_{2}\left(x_{4}-x_{3}\right)} e^{-i p_{3}\left(x_{5}-x_{4}\right)} J\left(x_{0}\right) J\left(x_{5}\right) \\
= & |J(p)|^{2}(2 \pi)^{3} \delta\left[\left(p_{1}-p-k_{1}\right)^{+}\right] \delta^{2}\left(\boldsymbol{p}_{1}-\boldsymbol{p}-\boldsymbol{k}_{1}\right) \\
& \times(2 \pi)^{3} \delta\left[\left(k_{1}-k-q\right)^{+}\right] \delta^{2}\left(\boldsymbol{k}_{1}-\boldsymbol{k}-\boldsymbol{q}\right) \\
& \times(2 \pi)^{3} \delta\left[\left(p+k-p_{2}\right)^{+}\right] \delta^{2}\left(\boldsymbol{p}+\boldsymbol{k}-\boldsymbol{p}_{2}\right)(2 \pi)^{3} \\
& \times \delta\left[\left(p+k+q-p_{2}\right)^{+}\right] \delta^{2}\left(\boldsymbol{p}+\boldsymbol{k}+\boldsymbol{q}-\boldsymbol{p}_{2}\right) I_{1}
\end{aligned}
$$

and where

$$
\begin{aligned}
I_{1}= & \int_{0}^{2 L} d x_{2}^{\prime+} e^{-\frac{i}{2}\left(k_{1}-k-q\right)^{-} x_{2}^{\prime+}} \int_{0}^{x_{2}^{\prime+}} d x_{1}^{\prime+} e^{-\frac{i}{2}\left(p_{1}-p-k_{1}\right)^{-} x_{1}^{\prime+}} \\
& \times \int_{0}^{\infty} d x_{3}^{\prime+} e^{-\frac{i}{2}\left(p+k-p_{2}\right)^{-} x_{3}^{\prime+}} \int_{0}^{2 L} d x_{4}^{\prime+} e^{-\frac{i}{2}\left(p+k+q-p_{3}\right)^{-} x_{4}^{\prime+}} \\
= & \frac{1}{-\frac{i}{2}\left(p_{1}-k_{1}-p\right)^{-}}\left[\frac{e^{-i\left(p_{1}-p-k-q\right)^{-} L}-1}{-\frac{i}{2}\left(p_{1}-p-k-q\right)^{-}}\right. \\
= & \frac{\left.-\frac{e^{-i\left(k_{1}-k-q\right)^{-} L}-1}{-\frac{i}{2}\left(k_{1}-k-q\right)^{-}}\right]}{-\frac{i}{2}\left(p_{1}-k_{1}-p\right)^{-}} \frac{e^{-i\left(p_{1}-p-k-q\right)^{-} L-1}}{-\frac{i}{2}\left(p_{1}-p-k-q\right)^{-}} \\
& \times\left[1-\frac{-\frac{i}{2}\left(p_{1}-p-k-q\right)^{-}}{e^{-i\left(p_{1}-p-k-q\right)^{-} L}-1} \frac{e^{-i\left(k_{1}-k-q\right)^{-} L}-1}{-\frac{i}{2}\left(k_{1}-k-q\right)^{-}}\right] .
\end{aligned}
$$

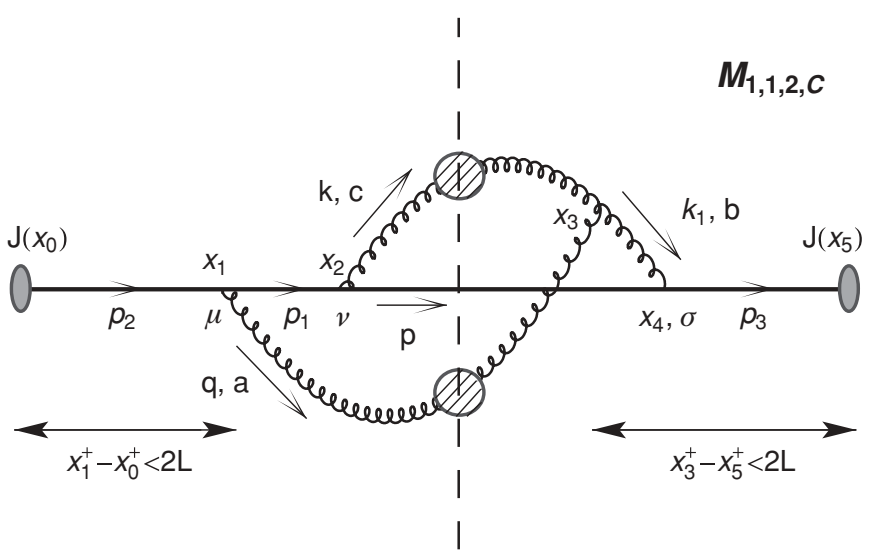

FIG. 7. Feynman diagrams $M_{1,1,1, C}$ and $M_{1,1,2, C}$ contributing to the radiative energy loss to first order in opacity, labeled the same way as in Fig. 1. 
By applying $\delta^{3}$ functions from Eq. (H2), and by using $p_{i}^{-}=$ $\frac{p_{i}^{2}+M^{2}}{p_{i}^{+}}$we obtain

$$
\begin{aligned}
& p_{1}^{-}=p_{3}^{-}=\frac{M^{2}}{p_{1}^{+}}=\frac{M^{2}}{(p+k+q)^{+}} \\
& p^{-}=\frac{(\boldsymbol{k}+\boldsymbol{q})^{2}+M^{2}}{p^{+}}, k^{-}=\frac{\boldsymbol{k}^{2}+m_{g}^{2}}{k^{+}}, \\
& k_{1}^{-}=\frac{(\boldsymbol{k}+\boldsymbol{q})^{2}+m_{g}^{2}}{(k+q)^{+}} p_{2}^{-}=\frac{\boldsymbol{q}^{2}+M^{2}}{(p+q)^{+}},
\end{aligned}
$$

which leads to

$$
\left(p+k-p_{2}\right)^{-}=\frac{\boldsymbol{k}^{2}+\chi}{2 x E} \equiv \xi
$$

[derived for $x=\frac{k^{+}}{\left(p+k^{+}\right)}$and $x \ll 1$ ] and

$$
\left(p_{1}-k_{1}-p\right)^{-} \approx-\frac{(\boldsymbol{k}+\boldsymbol{q})^{2}+\chi}{2 x E} \equiv-\zeta,
$$

[derived for $x_{1}=\frac{(k+q)^{+}}{(p+k+q)^{+}} \approx \frac{k}{(p+k)^{+}} \approx x$, and $x \ll 1$ ].

By using Eqs. (H5) and (H6) and assuming (as in previous sections) that finite-size effects are negligible for collisional contribution, we obtain

$$
\begin{aligned}
I_{1}= & \frac{-16 x^{2} E^{+2}}{\left(\boldsymbol{k}^{2}+\chi\right)\left[(\boldsymbol{k}+\boldsymbol{q})^{2}+\chi\right]} \\
& \times\left(1-\frac{e^{-i \zeta L}-1}{-i \zeta L}\right) 2 \pi L \delta\left(q_{1}-q_{z}\right),
\end{aligned}
$$

where we used $\delta\left[\left(p+k+q-p_{1}\right)^{-}\right] \approx \delta\left(q_{1}-q_{z}\right)$.

Let us now compute

$$
\begin{aligned}
\left(p_{1}\right. & \left.+p_{2}\right)^{\mu}\left(p+p_{2}\right)^{\nu}\left(p_{2}+p_{3}\right)^{\sigma} P_{\mu \rho_{1}}\left(k_{1}\right) P_{\rho_{3} v}(k) D_{\rho_{2} \sigma}^{>}(q) \\
& \times\left[g^{\rho_{1} \rho_{3}}\left(k+k_{1}\right)^{\rho_{2}}+g^{\rho_{2} \rho_{3}}(q-k)^{\rho_{1}}+g^{\rho_{1} \rho_{2}}\left(-k_{1}-q\right)^{\rho_{3}}\right] \\
\approx & {\left[\left(p_{1}+p_{2}\right)^{\mu} P_{\mu \rho_{1}}\left(k_{1}\right) P_{\nu}^{\rho_{1}}(k)\left(p+p_{2}\right)^{\nu}\right]\left[\left(k+k_{1}\right)^{\rho} D_{\rho \sigma}^{>}(q)\right.} \\
& \left.\times\left(p_{2}+p_{3}\right)^{\sigma}\right] \approx\left[-4 \frac{\boldsymbol{k} \cdot \boldsymbol{k}_{1}}{x^{2}}\right]\left\{\theta\left(1-\frac{q_{0}^{2}}{\overrightarrow{\mathbf{q}}^{2}}\right) f\left(q^{0}\right) E^{+} k^{+}\right. \\
& \left.\times \frac{\boldsymbol{q}^{2}}{\overrightarrow{\mathbf{q}}^{2}} 2 \operatorname{Im}\left[\frac{1}{q^{2}-\Pi_{L}(q)}-\frac{1}{q^{2}-\Pi_{T}(q)}\right]\right\} .
\end{aligned}
$$

(for more details see Eqs. (C1)-(C4) in Ref. [8]). Also, note that

$$
-i f^{a b c} t_{a} t_{b} t_{c}=\frac{1}{2}\left[t_{a}, t_{c}\right]\left[t_{c}, t_{a}\right] .
$$

Finally, by using Eqs. (H2), (H7), (H8), and (H9), Eq. (H1) reduces to

$$
\begin{aligned}
M_{1,1,1, C}= & -2 L \operatorname{Lg}^{4}\left[t_{a}, t_{c}\right]\left[t_{c}, t_{a}\right] \int \frac{d^{3} p}{(2 \pi)^{3} 2 E}|J(p)|^{2} \\
& \times \int \frac{d^{3} k}{(2 \pi)^{3} 2 \omega} \frac{d^{2} q}{(2 \pi)^{2}} \frac{\mu^{2}}{\boldsymbol{q}^{2}\left(\boldsymbol{q}^{2}+\mu^{2}\right)} \\
& \times \frac{\boldsymbol{k} \cdot(\boldsymbol{k}+\boldsymbol{q})}{\left(\boldsymbol{k}^{2}+\chi\right)\left[(\boldsymbol{k}+\boldsymbol{q})^{2}+\chi\right]} \\
& \times\left[1-\frac{\sin \zeta L}{\zeta L}+i \frac{1-\cos \zeta L}{\zeta L}\right]
\end{aligned}
$$

Because $M_{1,1,2, C}$ is a complex conjugate of $M_{1,1,1, C}$, one finally obtains

$$
\begin{aligned}
M_{1,1,1, C}+M_{1,1,2, C} \\
=-4 L T g^{4}\left[t_{a}, t_{c}\right]\left[t_{c}, t_{a}\right] \int \frac{d^{3} p}{(2 \pi)^{3} 2 E}|J(p)|^{2} \int \frac{d^{3} k}{(2 \pi)^{3} 2 \omega} \\
\quad \times \frac{d^{2} q}{(2 \pi)^{2}} \frac{\mu^{2}}{\boldsymbol{q}^{2}\left(\boldsymbol{q}^{2}+\mu^{2}\right)} \frac{\boldsymbol{k} \cdot(\boldsymbol{k}+\boldsymbol{q})}{\left(\boldsymbol{k}^{2}+\chi\right)\left[(\boldsymbol{k}+\boldsymbol{q})^{2}+\chi\right]} \\
\quad \times\left(1-\frac{\sin \zeta L}{\zeta L}\right) .
\end{aligned}
$$

\section{APPENDIX I: COMPUTATION OF DIAGRAMS \\ $M_{1,1,2, R}+M_{1,1,1, L}$}

In this appendix we will calculate cut diagrams $M_{1,1,1, L}$ and $M_{1,1,2, R}$, shown in Fig. 8. We start with $M_{1,1,2, R}$ :

$$
\begin{aligned}
M_{1,1,2, R}= & \int \prod_{i=0}^{5} d x_{i} J\left(x_{0}\right) \Delta_{++}^{+}\left(x_{1}-x_{0}\right) v_{\mu}^{+}\left(x_{1}\right) D_{++}^{+\mu \rho_{2}} \\
& \times\left(x_{3}-x_{1}\right) v_{\rho_{1} \rho_{2} \rho_{3}}^{+}\left(x_{3}\right) \Delta_{++}^{+}\left(x_{2}-x_{1}\right) \\
& \times D_{++}^{\rho_{1} v}\left(x_{3}-x_{2}\right) v_{v}^{+}\left(x_{2}\right) D_{-+}^{\rho_{3} \sigma}\left(x_{4}-x_{2}\right) v_{\sigma}^{-}\left(x_{4}\right) \\
& \times \Delta_{-+}\left(x_{4}-x_{2}\right) v_{--}^{-}\left(x_{5}-x_{4}\right) J\left(x_{5}\right) \\
& \times \theta\left(x_{1}^{+}-x_{0}^{+}\right) \theta\left(x_{2}^{+}-x_{1}^{+}\right) \theta\left[2 L-\left(x_{1}-x_{0}\right)^{+}\right] \\
& \times \theta\left(x_{3}^{+}-x_{2}^{+}\right) \theta\left(x_{4}^{+}-x_{5}^{+}\right) \\
= & \int_{-\infty}^{\infty} \int_{0}^{\infty} \prod_{i=1}^{\infty} \frac{d p_{i}^{+} d^{2} p_{i}}{(2 \pi)^{3} 2 p_{i}^{+}} \frac{d k_{1}^{+} d^{2} k_{1}}{(2 \pi)^{3} 2 k_{1}^{+}} \int \frac{d^{3} k}{(2 \pi)^{3} 2 \omega} \\
& \times \frac{d^{3} p}{(2 \pi)^{3} 2 E} \frac{d^{4} q}{(2 \pi)^{4}}(-i) g^{4} f^{a b c} t_{a} t_{b} t_{c}\left(p_{1}+p_{2}\right)^{\mu} \\
& \times\left(p+p_{1}\right)^{v}\left(p+p_{3}\right)^{\sigma} D_{\mu \rho_{2}}^{>}(q) P_{v \rho_{1}}\left(k_{1}\right) P_{\rho_{3} \sigma}(k) \\
& \times\left[g^{\rho_{1} \rho_{3}}\left(k+k_{1}\right)^{\rho_{2}}+g^{\rho_{2} \rho_{3}}(-k-q)^{\rho_{1}}\right. \\
& \left.+g^{\rho_{1} \rho_{2}}\left(q-k_{1}\right)^{\rho_{3}}\right] I,
\end{aligned}
$$

where

$$
\begin{aligned}
I= & \int \prod_{i=0}^{5} d x_{i} \theta\left(x_{1}^{+}-x_{0}^{+}\right) \theta\left(x_{2}^{+}-x_{1}^{+}\right) \theta\left(2 L-\left(x_{1}-x_{0}\right)^{+}\right) \\
& \times \theta\left(x_{3}^{+}-x_{2}^{+}\right) \theta\left(x_{4}^{+}-x_{5}^{+}\right) e^{-i p_{2}\left(x_{1}-x_{0}\right)} e^{-i q\left(x_{3}-x_{1}\right)} e^{-i p_{1}\left(x_{2}-x_{1}\right)} \\
& \times e^{-i k_{1}\left(x_{3}-x_{2}\right)} e^{-i k\left(x_{4}-x_{3}\right)} e^{-i p\left(x_{4}-x_{2}\right)} e^{-i p_{3}\left(x_{5}-x_{4}\right)} J\left(x_{0}\right) J\left(x_{5}\right) \\
= & |J(p)|^{2}(2 \pi)^{3} \delta\left[\left(p_{2}-p-k\right)^{+}\right] \delta^{2}\left(\boldsymbol{p}_{2}-\boldsymbol{p}-\boldsymbol{k}\right)(2 \pi)^{3} \\
& \times \delta\left[\left(p_{1}-p-k_{1}\right)^{+}\right] \delta^{2}\left(\boldsymbol{p}_{1}-\boldsymbol{p}-\boldsymbol{k}_{1}\right) \\
& \times(2 \pi)^{3} \delta\left[\left(k_{1}+q-k\right)^{+}\right] \delta^{2}\left(\boldsymbol{k}_{1}+\boldsymbol{q}-\boldsymbol{k}\right)(2 \pi)^{3} \\
& \times \delta\left[\left(p_{3}-p-k\right)^{+}\right] \delta^{2}\left(\boldsymbol{p}_{3}-\boldsymbol{p}-\boldsymbol{k}\right) I_{1}
\end{aligned}
$$

and where

$$
\begin{aligned}
I_{1}= & \int_{0}^{2 L} d x_{1}^{\prime+} e^{-\frac{i}{2}\left(p_{2}-p-k\right)^{-} x_{1}^{\prime+}} \int_{0}^{\infty} d x_{4}^{\prime+} e^{\frac{i}{2}\left(p_{3}-p-k\right)^{-} x_{4}^{\prime+}} \\
& \times \int_{0}^{\infty} d x_{3}^{\prime+} e^{-\frac{i}{2}\left(k_{1}+q-k\right)^{-} x_{3}^{\prime+}} \int_{0}^{x_{3}^{\prime+}} d x_{2}^{\prime+} e^{-\frac{i}{2}\left(p_{1}-p-k_{1}\right)^{-} x_{2}^{++}} .
\end{aligned}
$$




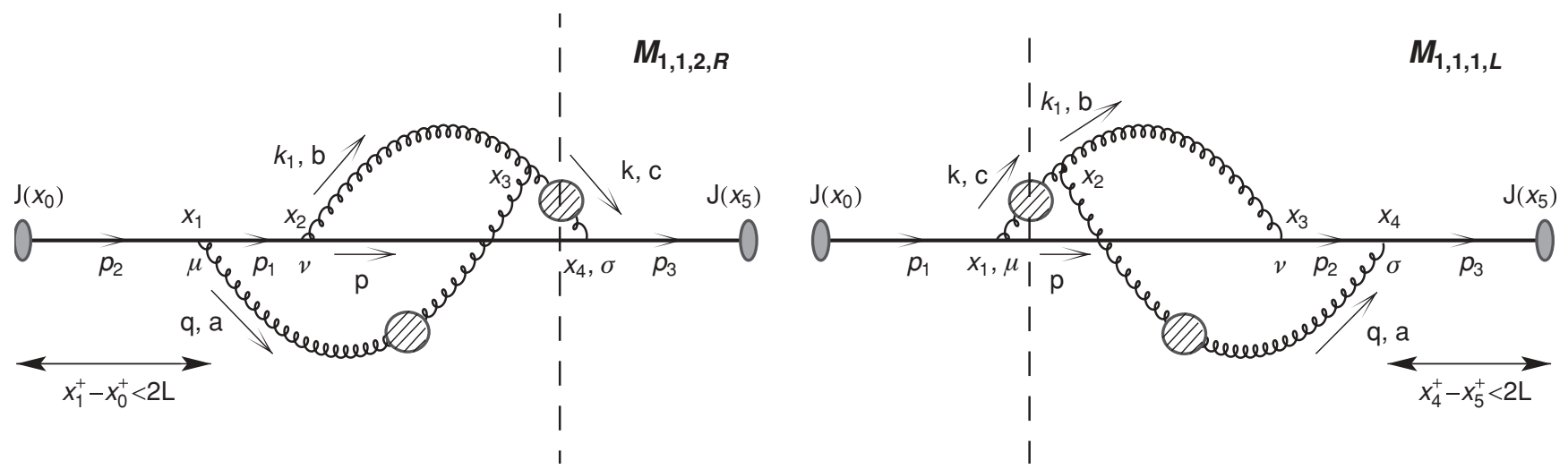

FIG. 8. Feynman diagrams $M_{1,1,1, L}$ and $M_{1,1,2, R}$ contributing to the radiative energy loss to first order in opacity, labeled the same way as in Fig. 1.

In the last equation we defined $x_{1}^{\prime}=x_{1}-x_{0}, x_{2}^{\prime}=x_{2}-x_{1}$, $x_{3}^{\prime}=x_{3}-x_{1}$, and $x_{4}^{\prime}=x_{4}-x_{5}$.

By applying $\delta^{3}$ functions from Eq. (I2), and by using $p_{i}^{-}=$ $\frac{p_{i}^{2}+M^{2}}{p_{i}^{+}}$, we obtain $p_{2}^{-}=p_{3}^{-}=\frac{M^{2}}{(p+k)^{+}}$, leading to (in soft-gluon, soft-rescattering approximation)

$$
\begin{aligned}
\left(p_{2}-p-k\right)^{-}= & \left(p_{3}-p-k\right)^{-}=-\xi \\
& \left(p_{1}-p-k_{1}\right)^{-} \approx-\zeta,
\end{aligned}
$$

where in the second relation we used $x_{1}=\frac{(k-q)^{+}}{(p+k-q)^{+}} \approx$ $\frac{k^{+}}{(p+k)^{+}}=x$.

$I_{1}$ then becomes

$$
I_{1}=\frac{8 L}{\xi \zeta} \frac{1-e^{i \xi L}}{i \xi L} \int_{0}^{\infty} d x_{3}^{\prime+}\left[e^{-\frac{i}{2}\left(q^{-}-\xi\right) x_{3}^{\prime+}}-e^{-\frac{i}{2}\left(q^{-}+\zeta-\xi\right) x_{3}^{\prime+}}\right]
$$
jets

Furthermore, similarly as in Ref. [8], for highly energetic

$$
\begin{aligned}
\{(p+ & \left.\left.p_{1}\right)^{v} P_{\nu \rho_{1}}\left(k_{1}\right) P_{\sigma}^{\rho_{1}}(k)\left(p+p_{3}\right)^{\sigma}\right\} \\
& \times\left\{\left(p_{1}+p_{2}\right)^{\mu} D_{\mu \sigma}(q)\left(k+k_{1}\right)^{\rho}\right\} \\
\approx & {\left[-4 \frac{\boldsymbol{k} \cdot(\boldsymbol{k}-\boldsymbol{q})}{x^{2}}\right]\left[E^{+} k^{+} \theta\left(1-\frac{q_{0}^{2}}{\overrightarrow{\mathbf{q}}^{2}}\right) f\left(q_{0}\right) \frac{\boldsymbol{q}^{2}}{\overrightarrow{\mathbf{q}}^{2}}\right.} \\
& \left.\times 2 \operatorname{Im}\left[\frac{1}{q^{2}-\Pi_{L}(q)}-\frac{1}{q^{2}-\Pi_{T}(q)}\right]\right] .
\end{aligned}
$$

By using Eqs. (I2), (I5), (I6), and (H9), Eq. (I1) finally reduces to

$$
\begin{aligned}
M_{1,1,2, R}= & L g^{4}\left[t_{a}, t_{c}\right]\left[t_{c}, t_{a}\right] \int \frac{d^{3} p}{(2 \pi)^{3} 2 E}|J(p)|^{2} \int \frac{d^{3} k}{(2 \pi)^{3} 2 \omega} \\
& \times \frac{d^{4} q}{(2 \pi)^{4}} \frac{\boldsymbol{k} \cdot(\boldsymbol{k}-\boldsymbol{q})}{\left(\boldsymbol{k}^{2}+\chi\right)\left[(\boldsymbol{k}-\boldsymbol{q})^{2}+\chi\right]} \theta\left(1-\frac{q_{0}^{2}}{\overrightarrow{\mathbf{q}}^{2}}\right) \\
& \times f\left(q_{0}\right) \frac{\boldsymbol{q}^{2}}{\overrightarrow{\mathbf{q}}^{2}} 2 \operatorname{Im}\left[\frac{1}{q^{2}-\Pi_{L}(q)}-\frac{1}{q^{2}-\Pi_{T}(q)}\right] \\
& \times\left(i \frac{1-\cos \xi L}{\xi L}+\frac{\sin \xi L}{\xi L}\right) \int_{0}^{\infty} d x_{3}^{\prime+} \\
& \times\left[e^{-\frac{i}{2}\left(q_{0}-q_{z}-\xi\right) x_{3}^{\prime+}}-e^{-\frac{i}{2}\left(q_{0}-q_{z}+\zeta-\xi\right) x_{3}^{\prime+}}\right]
\end{aligned}
$$

Because $M_{1,1,1, L}$ is a complex conjugate of $M_{1,1,2, R}$, it is straightforward to obtain $\left(y \equiv x_{3}^{+} / 2\right)$

$$
\begin{aligned}
& M_{1,1,1, L}+M_{1,1,2, R}=2 L^{4}\left[t_{a}, t_{c}\right]\left[t_{c}, t_{a}\right] \\
& \times \int \frac{d^{3} p}{(2 \pi)^{3} 2 E}|J(p)|^{2} \int \frac{d^{3} k}{(2 \pi)^{3} 2 \omega} \frac{d^{4} q}{(2 \pi)^{4}} \\
& \times \frac{\boldsymbol{k} \cdot(\boldsymbol{k}-\boldsymbol{q})}{\left(\boldsymbol{k}^{2}+\chi\right)\left[(\boldsymbol{k}-\boldsymbol{q})^{2}+\chi\right]} \theta\left(1-\frac{q_{0}^{2}}{\overrightarrow{\mathbf{q}}^{2}}\right) f\left(q_{0}\right) \frac{\boldsymbol{q}^{2}}{\overrightarrow{\mathbf{q}}^{2}} \\
& \times 2 \operatorname{Im}\left[\frac{1}{q^{2}-\Pi_{L}(q)}-\frac{1}{q^{2}-\Pi_{T}(q)}\right] \\
& \times\left\{\frac{\sin \xi L}{\xi L}\left[\delta\left(q_{0}-q_{z}-\xi\right)-\delta\left(q_{0}-q_{z}+\zeta-\xi\right)\right]\right\} \\
& +2 \frac{1-\cos \xi L}{\xi L} \int_{0}^{\infty} d y\left[\sin \left(q_{0}-q_{z}-\xi\right) y\right. \\
& \left.-\sin \left(q_{0}-q_{z}+\zeta-\xi\right) y\right] .
\end{aligned}
$$

By applying the same procedure as in Appendix F, we obtain $M_{1,1,1, L}+M_{1,1,2, R}=0$.

\section{APPENDIX J: COMPUTATION OF DIAGRAMS$$
M_{1,1,3, C} \text { AND } M_{1,1,4, C}
$$

In this appendix we will calculate the cut diagrams $M_{1,1,3, C}$ and $M_{1,1,4, C}$, shown in Fig. 9 . We start with $M_{1,1,3, C}$ :

$$
\begin{aligned}
M_{1,1,3, C}= & \int \prod_{i=0}^{5} d x_{i} J\left(x_{0}\right) \Delta_{++}^{+}\left(x_{1}-x_{0}\right) v_{\mu}^{+}\left(x_{1}\right) D_{++}^{+\mu \rho_{1}} \\
& \times\left(x_{2}-x_{1}\right) v_{\rho_{1} \rho_{2} \rho_{3}}^{+}\left(x_{2}\right) \Delta_{-+}\left(x_{3}-x_{1}\right) \\
& \times D_{-+}^{\rho_{2} \lambda}\left(x_{3}-x_{2}\right) v_{\lambda}^{-}\left(x_{3}\right) \Delta_{--}^{-}\left(x_{4}-x_{3}\right) D_{-+}^{\rho_{3} \sigma} \\
& \times\left(x_{4}-x_{2}\right) v_{\sigma}^{-}\left(x_{4}\right) \Delta_{--}^{-}\left(x_{5}-x_{4}\right) J\left(x_{5}\right) \\
& \times \int_{-\infty}^{\infty} \int_{0}^{\infty} \frac{d p_{3}^{+} d^{2} p_{3}}{(2 \pi)^{3} 2 p_{3}^{+}} e^{-i p_{3}\left(x_{5}-x_{4}\right)} \\
& \times\left[i g\left(p_{2}+p_{3}\right)^{\sigma} t_{b}\right] J\left(x_{5}\right) \theta\left(x_{1}^{+}-x_{0}^{+}\right) \theta\left(x_{2}^{+}-x_{1}^{+}\right)
\end{aligned}
$$




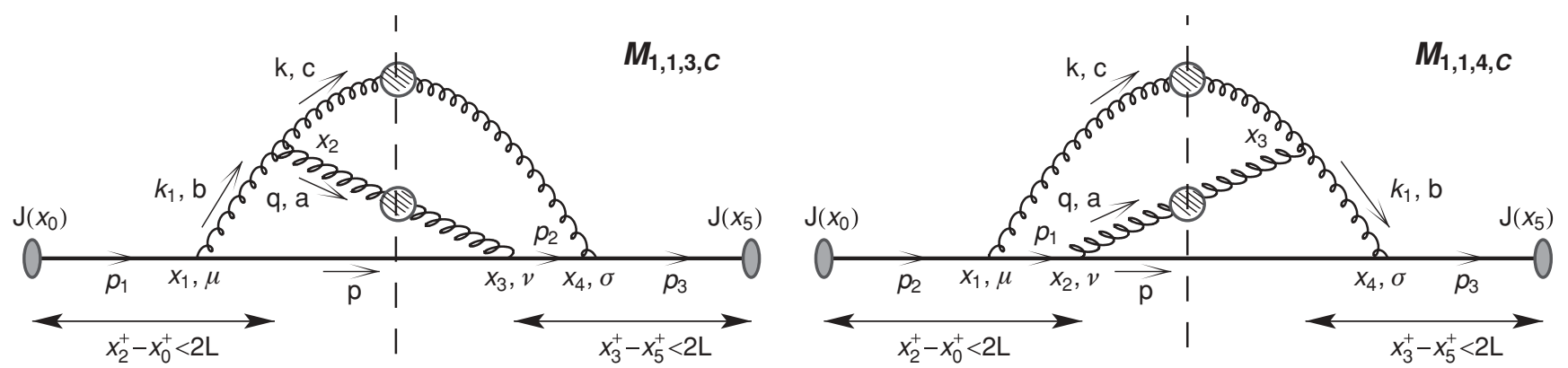

FIG. 9. Feynman diagrams $M_{1,1,3, C}$ and $M_{1,1,4, C}$ contributing to the radiative energy loss to first order in opacity, labeled the same way as in Fig. 1.

$$
\begin{aligned}
& \times \theta\left[2 L-\left(x_{2}-x_{0}\right)^{+}\right] \theta\left(x_{3}^{+}-x_{4}^{+}\right) \\
& \times \theta\left(x_{4}^{+}-x_{5}^{+}\right) \theta\left[2 L-\left(x_{3}-x_{5}\right)^{+}\right] \\
= & \int_{-\infty}^{\infty} \int_{0}^{\infty} \prod_{i=1}^{3} \frac{d p^{i+} d^{2} p^{i}}{(2 \pi)^{3} 2 p^{i+}} \frac{d k_{1}^{+} d^{2} k_{1}}{(2 \pi)^{3} 2 k_{1}^{+}} \int \frac{d^{3} k}{(2 \pi)^{3}} 2 \omega \\
& \times \frac{d^{3} p}{(2 \pi)^{3} 2 E} \frac{d^{4} q}{(2 \pi)^{4}} g^{4}(-i) f^{b a c} t_{b} t_{a} t_{c} \\
& \times\left(p+p_{1}\right)^{\mu}\left(p+p_{2}\right)^{v}\left(p_{2}+p_{3}\right)^{\sigma} \\
& \times P_{\mu \rho_{1}}\left(k_{1}\right) D_{\rho_{2} v}^{>}(q) P_{\rho_{3} \sigma}(k) \\
& \times\left(g^{\rho_{1} \rho_{3}}\left(k_{1}+k\right)^{\rho_{2}}+g^{\rho_{2} \rho_{3}}(q-k)^{\rho_{1}}\right. \\
& \left.+g^{\rho_{1} \rho_{2}}\left(-k_{1}-q\right)^{\rho_{3}}\right) I
\end{aligned}
$$

where

$$
\begin{aligned}
I= & \int \prod_{i=0}^{5} d x_{i} \theta\left(x_{1}^{+}-x_{0}^{+}\right) \theta\left(x_{2}^{+}-x_{1}^{+}\right) \theta\left(2 L-\left(x_{2}-x_{0}\right)^{+}\right) \\
& \times \theta\left(x_{3}^{+}-x_{4}^{+}\right) \theta\left(x_{4}^{+}-x_{5}^{+}\right) \theta\left[2 L-\left(x_{3}-x_{5}\right)^{+}\right] \\
& \times J\left(x_{0}\right) J\left(x_{5}\right) e^{-i p_{1}\left(x_{1}-x_{0}\right)} e^{-i k_{1}\left(x_{2}-x_{1}\right)} e^{-i p\left(x_{3}-x_{1}\right)} \\
& \times e^{-i q\left(x_{3}-x_{2}\right)} e^{-i p_{2}\left(x_{4}-x_{3}\right)} e^{-i p_{3}\left(x_{5}-x_{4}\right)} e^{-i k\left(x_{4}-x_{2}\right)} \\
= & |J(p)|^{2}(2 \pi)^{3} \delta\left[\left(p_{1}-p-k_{1}\right)^{+}\right] \delta^{2}\left(\boldsymbol{p}_{1}-\boldsymbol{p}-\boldsymbol{k}\right)(2 \pi)^{3} \\
& \times \delta\left[\left(k_{1}-k-q\right)^{+}\right] \delta^{2}\left(\boldsymbol{k}_{1}-\boldsymbol{k}-\boldsymbol{q}\right) \\
& \times(2 \pi)^{3} \delta\left[\left(p_{2}-p-q\right)^{+}\right] \delta^{2}\left(\boldsymbol{p}_{2}-\boldsymbol{p}-\boldsymbol{q}\right)(2 \pi)^{3} \\
& \times \delta\left[\left(p_{3}-p_{2}-k\right)^{+}\right] \delta^{2}\left(\boldsymbol{p}_{3}-\boldsymbol{p}_{2}-\boldsymbol{k}\right) I_{1}
\end{aligned}
$$

and where

$$
\begin{aligned}
I_{1}= & \int_{0}^{2 L} d x_{2}^{\prime+} e^{-\frac{i}{2}\left(k_{1}-k-q\right)^{-} x_{2}^{\prime+}} \int_{0}^{2 x_{2}^{\prime+}} d x_{1}^{\prime+} e^{-\frac{i}{2}\left(p_{1}-p-k_{1}\right)^{-} x_{1}^{\prime+}} \\
& \times \int_{0}^{2 L} d x_{3}^{\prime+} e^{-\frac{i}{2}\left(p_{2}-p-q\right)^{-} x_{3}^{\prime+}} \int_{0}^{2 x_{3}^{\prime+}} d x_{4}^{\prime+} e^{-\frac{i}{2}\left(p_{3}-p_{2}-k\right)^{-} x_{4}^{\prime+}} .
\end{aligned}
$$

Here we defined $x_{1}^{\prime+}=x_{1}-x_{0}, x_{2}^{\prime+}=x_{2}-x_{0}, x_{3}^{\prime+}=x_{3}-$ $x_{5}$, and $x_{4}^{\prime+}=x_{4}-x_{5}$.

By applying $\delta^{3}$ functions from Eq. (J1), and by using $p_{i}^{-}=$ $\frac{p_{i}^{2}+M^{2}}{p_{i}^{+}}$we obtain

$$
\begin{aligned}
& p^{-}=\frac{(\boldsymbol{k}+\boldsymbol{q})^{2}+M^{2}}{p^{+}}, p_{2}^{-}=\frac{\boldsymbol{k}^{2}+M^{2}}{(p+q)^{+}}, \\
& p_{3}^{-}=p_{1}^{-}=\frac{M^{2}}{p_{1}^{+}}=\frac{M^{2}}{(p+k+q)^{+}}, \\
& k^{-}=\frac{\boldsymbol{k}^{2}+m_{g}^{2}}{k^{+}}, k_{1}^{-}=\frac{(\boldsymbol{k}+\boldsymbol{q})^{2}+m_{g}^{2}}{(k+q)^{+}},
\end{aligned}
$$

which leads to

$$
\left(p_{1}-p-k_{1}\right)^{-}=-\zeta,
$$

[derived for $\left.x_{1}=\frac{(k+q)^{+}}{(p+k+q)^{+}} \approx \frac{k^{+}}{(p+k)^{+}} \approx x \ll 1\right]$ and

$$
\left(p_{3}-p_{2}-k\right)^{-}=-\xi \text {, }
$$

[derived for $x_{2}=\frac{k^{+}}{(p+k+q)^{+}} \approx \frac{(k+q)^{+}}{(p+k+q)^{+}} \approx \frac{k^{+}}{(p+k)^{+}} \approx x \ll 1$ ].

By using Eqs. (J4)-(J6), Eq. (J3) becomes

$$
\begin{aligned}
I_{1}= & \frac{16}{\zeta \xi}\left\{\frac{4 \sin ^{2}\left(p_{1}-p-k-q\right)^{-} L / 2}{\left[\left(p_{1}-p-k-q\right)^{-}\right]^{2}}\left[1-\frac{\left(p_{1}-p-k-q\right)^{-}}{e^{-i\left(p_{1}-p-k-q\right)^{-} L}-1} \times \frac{e^{-i\left(k_{1}-k-q\right)^{-} L}-1}{\left(k_{1}-k-q\right)^{-}}\right]-\frac{4 \sin ^{2}\left(p_{2}-p-q\right)^{-} L / 2}{\left[\left(p_{2}-p-q\right)^{-}\right]^{2}}\right. \\
& \left.\times\left[-\frac{\left(p_{2}-p-q\right)^{-}}{e^{-i\left(p_{2}-p-q\right)^{-} L}-1} \times \frac{e^{-i\left(k_{1}-k-q\right)^{-} L}-1}{\left(k_{1}-k-q\right)^{-}}+\frac{\left(p_{2}-p-q\right)^{-}}{e^{-i\left(p_{2}-p-q\right)^{-} L}-1} \times \frac{e^{-i\left(p_{1}-p-k-q\right)^{-} L}-1}{\left.p_{1}-p-k-q\right)^{-}}\right]\right\} \\
\approx & \frac{16}{\zeta \xi} 2 \pi L\left\{\delta\left(q^{-}+\zeta\right)\left(1-\frac{e^{-i \zeta L}-1}{-i \zeta L}\right)-\delta\left(p_{1}-p-q\right)^{-}\left[\frac{e^{i \xi L}-1}{i \xi L}-\frac{e^{-i(\zeta-\xi) L}-1}{-i(\zeta-\xi) L}\right]\right\} \\
\approx & \frac{16}{\zeta \xi} 2 \pi L \delta\left(q^{-}\right)\left[1-\frac{e^{-i \zeta L}-1}{-i \zeta L}-\frac{e^{i \xi L}-1}{i \xi L}+\frac{e^{-i(\zeta-\xi) L}-1}{-i(\zeta-\xi) L}\right],
\end{aligned}
$$


where, as in the previous sections, we assumed that finite-size effects are negligible for collisional contribution, and in the last step we used Eq. (A9).

Analogously to calculations performed in Ref. [8] (see Appendix C in Ref. [8]) one has

$$
\begin{aligned}
(p+ & \left.p_{1}\right)^{\mu}\left(p+p_{2}\right)^{\nu}\left(p_{2}+p_{3}\right)^{\sigma} P_{\mu \rho_{1}}\left(k_{1}\right) D_{\rho_{2} \lambda}^{>}(q) P_{\rho_{3} \sigma}(k) \\
& \times\left(g^{\rho_{1} \rho_{3}}\left(k+k_{1}\right)^{\rho_{2}}+g^{\rho_{2} \rho_{3}}(q-k)^{\rho_{1}}+g^{\rho_{1} \rho_{2}}\left(-k_{1}-q\right)^{\rho_{3}}\right) \\
\approx & \left(p+p_{1}\right)^{\mu} P_{\mu \rho_{1}}\left(k_{1}\right) P_{\sigma}^{\rho_{1}}(k)\left(p_{2}+p_{3}\right)^{\sigma} \\
& \times\left[\left(k+k_{1}\right)^{\rho} D_{\rho \sigma}^{>}(q)\left(p+p_{2}\right)^{\nu}\right] \\
\approx & -4 \frac{\boldsymbol{k} \cdot(\boldsymbol{k}+\boldsymbol{q})}{x^{2}} \theta\left(1-\frac{q_{0}^{2}}{\overrightarrow{\mathbf{q}}^{2}}\right) f\left(q^{0}\right) E^{+} k^{+} \frac{\boldsymbol{q}^{2}}{\overrightarrow{\mathbf{q}}^{2}} \\
& \times 2 \operatorname{Im}\left[\frac{1}{q^{2}-\Pi_{L}(q)}-\frac{1}{q^{2}-\Pi_{T}(q)}\right] .
\end{aligned}
$$

By using Eqs. (J2), (J7), (J8), and (H9), Eq. (J1) finally reduces to

$$
\begin{aligned}
M_{1,1,3, C}= & -2 L T g^{4}\left[t_{a}, t_{c}\right]\left[t_{c}, t_{a}\right] \int \frac{d^{3} p}{(2 \pi)^{3} 2 E}|J(p)|^{2} \\
& \times \int \frac{d^{3} k}{(2 \pi)^{3} 2 \omega} \frac{d^{2} q}{(2 \pi)^{2}} \frac{\mu^{2}}{\boldsymbol{q}^{2}\left(\boldsymbol{q}^{2}+\mu^{2}\right)} \\
& \times \frac{\boldsymbol{k} \cdot(\boldsymbol{k}+\boldsymbol{q})}{\left(\boldsymbol{k}^{2}+\chi\right)\left[(\boldsymbol{k}+\boldsymbol{q})^{2}+\chi\right]} \\
& \times\left[1-\frac{e^{-i \zeta L}-1}{-i \zeta L}-\frac{e^{i \xi L}-1}{i \xi L}+\frac{e^{-i(\zeta-\xi) L}-1}{-i(\zeta-\xi) L}\right] .
\end{aligned}
$$

Because $M_{1,1,4, C}$ is a complex conjugate of $M_{1,1,3, C}$, one finally obtains

$$
\begin{aligned}
& M_{1,1,3, C}+M_{1,1,4, C} \\
& \quad=-4 \operatorname{Lg}^{4}\left[t_{a}, t_{c}\right]\left[t_{c}, t_{a}\right] \int \frac{d^{3} p}{(2 \pi)^{3} 2 E}|J(p)|^{2} \int \frac{d^{3} k}{(2 \pi)^{3} 2 \omega}
\end{aligned}
$$
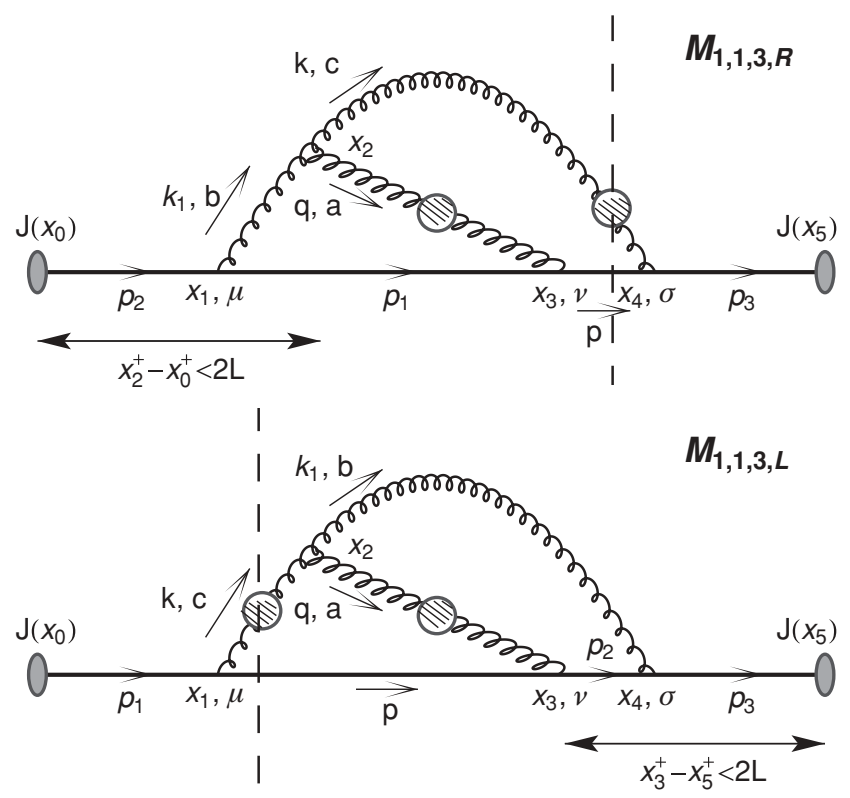

$$
\begin{aligned}
& \times \frac{d^{2} q}{(2 \pi)^{2}} \frac{\mu^{2}}{\boldsymbol{q}^{2}\left(\boldsymbol{q}^{2}+\mu^{2}\right)} \frac{\boldsymbol{k} \cdot(\boldsymbol{k}+\boldsymbol{q})}{\left(\boldsymbol{k}^{2}+\chi\right)\left[(\boldsymbol{k}+\boldsymbol{q})^{2}+\chi\right]} \\
& \times\left[1-\frac{\sin \zeta L}{\zeta L}-\frac{\sin \xi L}{\xi L}+\frac{\sin (\zeta-\xi) L}{(\zeta-\xi) L}\right] .
\end{aligned}
$$

\section{APPENDIX K: COMPUTATION OF DIAGRAMS $M_{1,1,3, R}, M_{1,1,3, L}, M_{1,1,4, L}$, AND $M_{1,1,4, R}$}

In this appendix we will calculate the cut diagrams $M_{1,1,3, R}$, $M_{1,1,3, L}, M_{1,1,4, R}$, and $M_{1,1,4, L}$ shown in Fig. 10 . We start with $M_{1,1,3, R}$ :

$$
\begin{aligned}
M_{1,1,3, R}= & \int \prod_{i=0}^{5} d x_{i} J\left(x_{0}\right) \Delta_{++}^{+}\left(x_{1}-x_{0}\right) v_{\mu}^{+}\left(x_{1}\right) D_{++}^{+\mu \rho_{1}} \\
& \times\left(x_{2}-x_{1}\right) v_{\rho_{1} \rho_{2} \rho_{3}}^{+}\left(x_{2}\right) \Delta_{++}^{+}\left(x_{3}-x_{1}\right) \\
& \times D_{++}^{+\rho_{2} v}\left(x_{3}-x_{2}\right) v_{v}^{+}\left(x_{3}\right) D_{-+}^{\rho_{3} \sigma}\left(x_{4}-x_{2}\right) v_{\sigma}^{-}\left(x_{4}\right) \\
& \times \Delta_{-+}\left(x_{4}-x_{3}\right) \Delta_{--}^{-}\left(x_{5}-x_{4}\right) J\left(x_{5}\right) \\
& \times \theta\left(x_{1}^{+}-x_{0}^{+}\right) \theta\left(x_{2}^{+}-x_{1}^{+}\right) \theta\left[2 L-\left(x_{2}-x_{0}\right)^{+}\right] \\
& \times \theta\left(x_{3}^{+}-x_{2}^{+}\right) \theta\left(x_{4}^{+}-x_{5}^{+}\right) \\
\approx & -\int_{-\infty}^{\infty} \int_{0}^{\infty} \prod_{i=1}^{3} \frac{d p_{i}^{+} d^{2} p_{i}}{(2 \pi)^{3} 2 p_{i}^{+}} \frac{d k_{1}^{+} d^{2} k_{1}}{(2 \pi)^{3} 2 k_{1}^{+}} \int \frac{d^{3} k}{(2 \pi)^{3}} 2 \omega \\
& \times \frac{d^{3} p}{(2 \pi)^{3} 2 E} \frac{d^{4} q}{(2 \pi)^{4}}(-i) g^{4} f^{c b a} t_{c} t_{b} t_{a} \\
& \times\left[\left(p_{1}+p_{2}\right)^{\mu} P_{\mu \rho_{1}}\left(k_{1}\right) P_{\sigma}^{\rho_{1}}(k)\left(p+p_{3}\right)^{\sigma}\right] \\
& \times\left[\left(k+k_{1}\right)^{\rho_{2}} D_{\rho_{2} \sigma}^{>}(q)\left(p+p_{1}\right)^{\nu}\right] I, \quad(\mathrm{~K} 1)
\end{aligned}
$$

FIG. 10. Feynman diagrams $M_{1,1,3, R}, M_{1,1,3, L}, M_{1,1,4, R}$, and $M_{1,1,4, L}$ contributing to the radiative energy loss to first order in opacity, labeled the same way as in Fig. 1. 
where we used that in soft-gluon, soft-rescattering approximation (see Eqs. (C1)-(C4) in Eq. [8])

$$
\begin{aligned}
\left(p_{1}\right. & \left.+p_{2}\right)^{\mu}\left(p+p_{1}\right)^{v}\left(p+p_{3}\right)^{\sigma} P_{\mu \rho_{1}}\left(k_{1}\right) D_{\rho_{2} v}^{>}(q) P_{\rho_{3} \sigma}(k) \\
& \times\left(g^{\rho_{1} \rho_{3}}\left(k_{1}+k\right)^{\rho_{2}}+g^{\rho_{2} \rho_{3}}(q-k)^{\rho_{1}}+g^{\rho_{1} \rho_{2}}\left(-k_{1}-q\right)^{\rho_{3}}\right) \\
\approx & {\left[\left(p_{1}+p_{2}\right)^{\mu} P_{\mu \rho_{1}}\left(k_{1}\right) P_{\sigma}^{\rho_{1}}(k)\left(p+p_{3}\right)^{\sigma}\right] } \\
& \times\left[\left(k+k_{1}\right)^{\rho_{2}} D_{\rho_{2} v}^{>}(q)\left(p+p_{1}\right)^{v}\right] .
\end{aligned}
$$

In Eq. (K1) $I$ corresponds to

$$
\begin{aligned}
I= & \int \prod_{i=0}^{5} d x_{i} \theta\left(x_{1}^{+}-x_{0}^{+}\right) \theta\left(x_{2}^{+}-x_{1}^{+}\right) \theta\left(2 L-\left(x_{2}-x_{0}\right)^{+}\right) \\
& \times \theta\left(x_{3}^{+}-x_{2}^{+}\right) \theta\left(x_{4}^{+}-x_{5}^{+}\right) \\
& \times e^{-i p_{2}\left(x_{1}-x_{0}\right)} e^{-i k_{1}\left(x_{2}-x_{1}\right)} e^{-i p_{1}\left(x_{3}-x_{1}\right)} e^{-i q\left(x_{3}-x_{2}\right)} \\
& \times e^{-i k\left(x_{4}-x_{2}\right)} e^{-i p\left(x_{4}-x_{3}\right)} e^{-i p_{3}\left(x_{5}-x_{4}\right)} J\left(x_{0}\right) J\left(x_{5}\right) \\
= & |J(p)|^{2}(2 \pi)^{3} \delta\left[\left(p_{2}-p_{1}-k_{1}\right)^{+}\right] \delta^{2}\left(\boldsymbol{p}_{2}-\boldsymbol{p}_{1}-\boldsymbol{k}_{1}\right) \\
& \times(2 \pi)^{3} \delta\left[\left(k_{1}-k+p_{1}-p\right)^{+}\right] \delta^{2}\left(\boldsymbol{k}_{1}-\boldsymbol{k}+\boldsymbol{p}_{1}-\boldsymbol{p}\right) \\
& \times(2 \pi)^{3} \delta\left[\left(p_{1}+q-p\right)^{+}\right] \delta^{2}\left(\boldsymbol{p}_{1}+\boldsymbol{q}-\boldsymbol{p}\right)(2 \pi)^{3} \\
& \times \delta\left[\left(p_{3}-p-k\right)^{+}\right] \delta^{2}\left(\boldsymbol{p}_{3}-\boldsymbol{p}-\boldsymbol{k}\right) I_{1},
\end{aligned}
$$

where

$$
\begin{aligned}
I_{1}= & \int_{0}^{2 L} d x_{2}^{\prime+} e^{-\frac{i}{2}\left(k_{1}-k-p_{1}-p\right)^{-} x_{2}^{\prime+}} \int_{0}^{x_{2}^{\prime+}} d x_{1}^{\prime+} e^{-\frac{i}{2}\left(p_{2}-p_{1}-k_{1}\right)^{-} x_{1}^{\prime+}} \\
& \times \int_{0}^{\infty} d x_{3}^{\prime+} e^{-\frac{i}{2}\left(p_{1}+q-p\right)^{-} x_{3}^{\prime+}} \int_{0}^{\infty} d x_{4}^{\prime+} e^{\frac{i}{2}\left(p_{3}-p-k\right)^{-} x_{4}^{\prime+}} .
\end{aligned}
$$

By applying $\delta^{3}$ functions from Eq. (K3) and by using $p_{i}^{-}=\frac{p_{i}^{2}+M^{2}}{p_{i}^{+}}$we obtain in the soft-gluon, soft-rescattering approximation [see also Eq. (A9)]

$$
\begin{aligned}
& p_{2}^{-}=p_{3}^{-}=\frac{M^{2}}{E^{+}}, \quad\left(p_{2}-p_{1}-k_{2}\right)^{-}=-\zeta, \\
& \left(p_{3}-p-k\right)^{-}=\left(p_{2}-p-k\right)^{-}=-\xi, \\
& \left(k_{1}-k+p_{1}-p\right)^{-}=\zeta-\xi, \quad\left(q+p_{1}-p\right)^{-} \approx q_{0}-q_{z} .
\end{aligned}
$$

By using Eq. (K5), Eq. (K4) becomes $\left(y \equiv \frac{x_{3}^{\prime+}}{2}\right)$

$$
I_{1}=-\frac{16 L}{\zeta \xi}\left[\frac{e^{i \xi L}-1}{i \xi L}-\frac{e^{i(\xi-\zeta) L}-1}{i(\xi-\zeta) L}\right] \int_{0}^{\infty} e^{-i q^{-} y} d y .
$$

Similarly to previous appendices, Eq. (K2) is equal to

$$
\begin{aligned}
\left\{\left(p_{1}\right.\right. & \left.\left.+p_{2}\right)^{\mu} P_{\mu \rho_{1}}\left(k_{1}\right) P_{\sigma}^{\rho_{1}}(k)\left(p+p_{2}\right)^{\sigma}\right\} \\
& \times\left\{\left(k+k_{1}\right)^{\rho_{2}} D_{\rho_{2} \nu}^{>}(q)\left(p_{2}+p_{3}\right)^{\nu}\right\} \\
\approx & -4 \frac{\boldsymbol{k} \cdot(\boldsymbol{k}+\boldsymbol{q})}{x^{2}} \theta\left(1-\frac{q_{0}^{2}}{\overrightarrow{\mathbf{q}}^{2}}\right) f\left(q^{0}\right) E^{+} k^{+} \frac{\boldsymbol{q}^{2}}{\overrightarrow{\mathbf{q}}^{2}} \\
\quad & 2 \operatorname{Im}\left[\frac{1}{q^{2}-\Pi_{L}(q)}-\frac{1}{q^{2}-\Pi_{T}(q)}\right] .
\end{aligned}
$$

Finally, by using Eqs. (K3), (K6)-(H9), Eq. (K1) reduces to $M_{1,1,3, R}$

$$
=-2 L g^{4}\left[t_{a}, t_{c}\right]\left[t_{c}, t_{a}\right] \int \frac{d^{3} p}{(2 \pi)^{3} 2 E}|J(p)|^{2} \int \frac{d^{3} k}{(2 \pi)^{3} 2 \omega}
$$

$$
\begin{aligned}
& \times \frac{d^{4} q}{(2 \pi)^{4}} \frac{\boldsymbol{k} \cdot(\boldsymbol{k}+\boldsymbol{q})}{\left[(\boldsymbol{k}+\boldsymbol{q})^{2}+\chi\right]\left(\boldsymbol{k}^{2}+\chi\right)} \theta\left(1-\frac{q_{0}^{2}}{\overrightarrow{\mathbf{q}}^{2}}\right) f\left(q_{0}\right) \frac{\boldsymbol{q}^{2}}{\overrightarrow{\mathbf{q}}^{2}} \\
& \times 2 \operatorname{Im}\left[\frac{1}{q^{2}-\Pi_{L}(q)}-\frac{1}{q^{2}-\Pi_{T}(q)}\right] \\
& \times\left[\frac{e^{i \xi L}-1}{i \xi L}-\frac{e^{i(\xi-\zeta) L}-1}{i(\xi-\zeta) L}\right] \int_{0}^{\infty} e^{-i q^{-y}} d y .
\end{aligned}
$$

Because $M_{1,1,4, L}$ is a complex conjugate of $M_{1,1,3, R}$, one finally obtains

$$
\begin{aligned}
M_{1,1,3, R}+M_{1,1,4, L} \\
=-2 L T g^{4}\left[t_{a}, t_{c}\right]\left[t_{c}, t_{a}\right] \int \frac{d^{3} p}{(2 \pi)^{3} 2 E}|J(p)|^{2} \int \frac{d^{3} k}{(2 \pi)^{3} 2 \omega} \\
\times \frac{d^{2} q}{(2 \pi)^{2}} \frac{\mu^{2}}{\boldsymbol{q}^{2}\left(\boldsymbol{q}^{2}+\mu^{2}\right)} \frac{\boldsymbol{k} \cdot(\boldsymbol{k}+\boldsymbol{q})}{\left[(\boldsymbol{k}+\boldsymbol{q})^{2}+\chi\right]\left(\boldsymbol{k}^{2}+\chi\right)} \\
\times\left[\frac{\sin \xi L}{\xi L}-\frac{\sin (\zeta-\xi) L}{(\zeta-\xi) L}\right] .
\end{aligned}
$$

Similarly $M_{1,1,3, L}+M_{1,1,4, R}=M_{1,1,3, R}+M_{1,1,4, L}$ leading to

$$
\begin{aligned}
M_{1,1,3, L}+M_{1,1,3, R}+M_{1,1,4, R}+M_{1,1,4, L} \\
=-4 L T g^{4}\left[t_{a}, t_{c}\right]\left[t_{c}, t_{a}\right] \int \frac{d^{3} p}{(2 \pi)^{3} 2 E}|J(p)|^{2} \int \frac{d^{3} k}{(2 \pi)^{3} 2 \omega} \\
\times \frac{d^{2} q}{(2 \pi)^{2}} \frac{\mu^{2}}{\boldsymbol{q}^{2}\left(\boldsymbol{q}^{2}+\mu^{2}\right)} \frac{\boldsymbol{k} \cdot(\boldsymbol{k}+\boldsymbol{q})}{\left[(\boldsymbol{k}+\boldsymbol{q})^{2}+\chi\right]\left(\boldsymbol{k}^{2}+\chi\right)} \\
\quad \times\left[\frac{\sin \xi L}{\xi L}-\frac{\sin (\zeta-\xi) L}{(\zeta-\xi) L}\right] .
\end{aligned}
$$

\section{APPENDIX L: COMPUTATION OF DIAGRAM $M_{1,2, C}$}

In Appendices $\mathrm{L}$ and $\mathrm{M}$ we present in some detail the calculation of the diagrams where both ends of the exchanged gluon $q$ are attached to the radiated gluon $k$, i.e., there are two three-gluon vertices involved in the process. In this appendix, we start with the calculation of the diagram $M_{1,2, C}$ shown in Fig. 11.

$$
\begin{aligned}
& M_{1,2, C} \\
& \quad=\int \prod_{i=0}^{5} d x_{i} J\left(x_{0}\right) \Delta_{++}^{+}\left(x_{1}-x_{0}\right) v_{\mu}^{+}\left(x_{1}\right) D_{++}^{+\mu \rho_{1}}\left(x_{2}-x_{1}\right)
\end{aligned}
$$

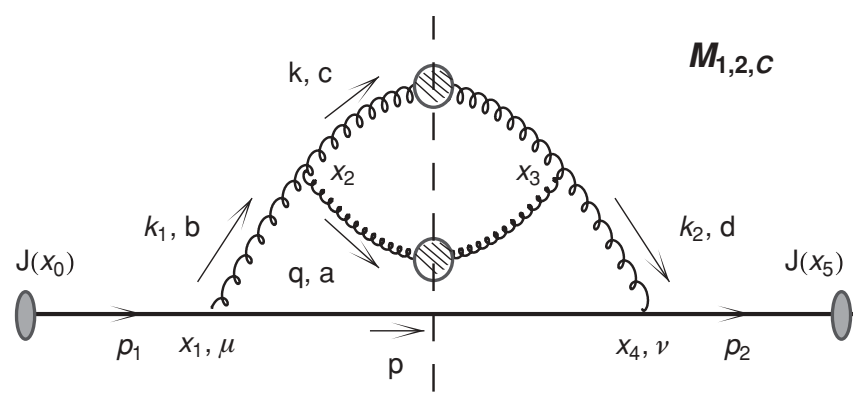

FIG. 11. Feynman diagram $M_{1,2}$ contributing to the radiative energy loss to first order in opacity, labeled the same way as in Fig. 1. 


$$
\begin{aligned}
& \times v_{\rho_{1} \rho_{2} \rho_{3}}^{+}\left(x_{2}\right) D_{-+}^{\rho_{3} \sigma_{3}}\left(x_{3}-x_{2}\right) D_{-+}^{\rho_{2} \sigma_{2}}\left(x_{3}-x_{2}\right) \Delta_{-+}\left(x_{4}-x_{1}\right) \\
& \times v_{\sigma_{1} \sigma_{2} \sigma_{3}}^{-}\left(x_{3}\right) D_{--}^{-\sigma_{1} v}\left(x_{4}-x_{3}\right) v_{v}^{-}\left(x_{4}\right) \Delta_{--}^{-}\left(x_{5}-x_{4}\right) J\left(x_{5}\right) \\
& \times \theta\left(x_{1}^{+}-x_{0}^{+}\right) \theta\left(x_{2}^{+}-x_{1}^{+}\right) \theta\left[2 L-\left(x_{2}-x_{0}\right)^{+}\right] \\
& \times \theta\left(x_{3}^{+}-x_{4}^{+}\right) \theta\left(x_{4}^{+}-x_{5}^{+}\right) \theta\left[2 L-\left(x_{3}-x_{5}\right)^{+}\right] \\
& =-\int_{0}^{\infty} \int_{-\infty}^{\infty} \prod_{i=1}^{2} \frac{d p_{i}^{+} d^{2} p_{i}}{(2 \pi)^{3} 2 p_{i}^{+}} \frac{d k_{i}^{+} d^{2} k_{i}}{(2 \pi)^{3} 2 k_{i}^{+}} \int \frac{d^{3} k}{(2 \pi)^{3} 2 \omega} \\
& \times \frac{d^{3} p}{(2 \pi)^{3} 2 E} \frac{d^{4} q}{(2 \pi)^{4}} g^{4} f^{b a c} t_{b} f^{d a c} t_{d} \\
& \times\left[\left(k+k_{1}\right)^{\rho_{2}} D_{\rho_{2} \sigma_{2}}^{-}\left(k+k_{2}\right)^{\sigma_{2}}\right]\left[\left(p+p_{1}\right)^{\mu} P_{\mu \rho_{1}}\left(k_{1}\right)\right. \\
& \left.\times P^{\sigma_{1} \rho_{1}}(k) P_{\rho_{1} v}\left(k_{2}\right)\left(p+p_{2}\right)^{v}\right] I,
\end{aligned}
$$

where in the second step we only keep the dominant parts from triple gluon vertices (see Ref. [8]).

In Eq. (L1) $I$ corresponds to

$$
\begin{aligned}
I= & \int \prod_{i=0}^{5} d x_{i} \theta\left(x_{1}^{+}-x_{0}^{+}\right) \theta\left(x_{2}^{+}-x_{1}^{+}\right) \theta\left(2 L-\left(x_{2}-x_{0}\right)^{+}\right) \\
& \times \theta\left[2 L-\left(x_{3}-x_{5}\right)^{+}\right] \theta\left(x_{3}^{+}-x_{4}^{+}\right) \theta\left(x_{4}^{+}-x_{5}^{+}\right)
\end{aligned}
$$

$$
\begin{aligned}
& \times e^{-i p_{1}\left(x_{1}-x_{0}\right)} e^{-i k_{1}\left(x_{2}-x_{1}\right)} e^{-i k\left(x_{3}-x_{2}\right)} e^{-i q\left(x_{3}-x_{2}\right)} \\
& \times e^{-i p\left(x_{4}-x_{1}\right)} e^{-i k_{2}\left(x_{4}-x_{3}\right)} e^{-i p_{2}\left(x_{5}-x_{4}\right)} J\left(x_{0}\right) J\left(x_{5}\right) \\
= & |J(p)|^{2}(2 \pi)^{3} \delta\left[\left(p_{1}-p-k_{1}\right)^{+}\right] \delta^{2}\left(\boldsymbol{p}_{1}-\boldsymbol{p}-\boldsymbol{k}_{1}\right)(2 \pi)^{3} \delta \\
& \times\left[\left(k_{1}-k-q\right)^{+}\right] \delta^{2}\left(\boldsymbol{k}_{1}-\boldsymbol{k}-\boldsymbol{q}\right) \\
& \times(2 \pi)^{3} \delta\left[\left(k_{2}-q-k\right)^{+}\right] \delta^{2}\left(\boldsymbol{k}_{2}-\boldsymbol{q}-\boldsymbol{k}\right)(2 \pi)^{3} \delta \\
& \times\left[\left(p_{2}-p-k_{2}\right)^{+}\right] \delta^{2}\left(\boldsymbol{p}_{2}-\boldsymbol{p}-\boldsymbol{k}_{2}\right) I_{1},
\end{aligned}
$$

where

$$
\begin{aligned}
I_{1}= & \int_{0}^{2 L} d x_{2}^{\prime+} e^{-\frac{i}{2}\left(k_{1}-k-q\right)^{-} x_{2}^{\prime+}} \int_{0}^{x_{2}^{\prime+}} d x_{1}^{\prime+} e^{-\frac{i}{2}\left(p_{1}-p-k_{1}\right)^{-} x_{1}^{\prime+}} \\
& \times \int_{0}^{2 L} d x_{3}^{\prime+} e^{\frac{i}{2}\left(k_{2}-q-k\right)^{-} x_{3}^{\prime+}} \int_{0}^{x_{3}^{\prime+}} d x_{4}^{\prime+} e^{\frac{i}{2}\left(p_{2}-p-k_{2}\right)^{-} x_{4}^{\prime+}} .
\end{aligned}
$$

Here $x_{1}^{\prime+}=x_{1}^{+}-x_{0}^{+}, x_{2}^{\prime+}=x_{2}^{+}-x_{0}^{+}, x_{3}^{\prime+}=x_{3}^{+}-x_{5}^{+}$, and $x_{4}^{\prime+}=x_{4}^{+}-x_{5}^{+}$. Furthermore, from $\delta$ functions in Eq. (L2) it follows that

$$
\begin{aligned}
p_{2} & =p_{1}, \quad k_{2}=k_{1}, \\
\left(p_{1}-k_{1}-p\right)^{-} & =\left(p_{2}-k_{2}-p\right)^{-}=-\zeta .
\end{aligned}
$$

After using Eq. (L4), Eq. (L3) becomes

$$
\begin{aligned}
I_{1}= & \frac{16}{\zeta^{2}}\left(\frac { 4 \operatorname { s i n } ^ { 2 } [ ( p _ { 1 } - p - k - q ) ^ { - } L / 2 ] - 1 } { [ ( p _ { 1 } - p - k - p ) ^ { - } ] ^ { 2 } } \left\{1-\frac{\left(p_{1}-p-k-q\right)^{-}}{e^{i\left(p_{1}-p-k-q\right)^{-} L}-1} \times \frac{e^{-i\left(k_{1}-k-q\right)^{-} L}-1}{k_{1}-k-q}-\frac{\left(p_{1}-p-k-q\right)^{-}}{e^{-i\left(p_{1}-p-k-q\right)^{-} L}-1}\right.\right. \\
& \left.\left.\times \frac{e^{i\left(k_{1}-k-q\right)^{-} L}-1}{k_{1}-k-q}\right\}+\frac{4 \sin ^{2}\left[\left(k_{1}-k-q\right)^{-} L / 2\right]-1}{\left[\left(k_{1}-k-q\right)^{-}\right]^{2}}\right) \approx \frac{16}{\zeta^{2}}\left\{2 \pi L \delta\left[\left(p_{1}-p-k-q\right)^{-}\right]\left(1-\frac{e^{i \zeta L}-1}{i \zeta L}-\frac{e^{-i \zeta L}-1}{-i \zeta L}\right)\right. \\
& \left.+2 \pi L \delta\left[\left(k_{1}-k-q\right)^{-}\right]\right\}=2 \pi L \frac{16}{\zeta^{2}}\left\{\left(1-2 \frac{\sin \zeta L}{\zeta L}\right) \delta\left[\left(p_{1}-p-k-q\right)^{-}\right]+\delta\left[\left(k_{1}-k-q\right)^{-}\right]\right\} \\
\approx & \frac{64 \pi L}{\zeta^{2}}\left(1-\frac{\sin \zeta L}{\zeta L}\right) \delta\left(q_{0}-q_{z}\right) .
\end{aligned}
$$

As in the previous sections, we here assumed that finite-size effects are negligible for collisional energy loss. Furthermore, in the last step we used Eq. (A9), i.e., $\left(p_{1}-p-k-q\right)^{-} \approx$ $\left(k_{1}-k-q\right)^{-} \approx q_{0}-q_{z}$.

Similarly as in Ref. [8], for highly energetic jets

$$
\begin{aligned}
& \left(p+p_{1}\right)^{\mu} P_{\mu \rho_{1}}\left(k_{1}\right) P^{\sigma_{1} \rho_{1}}(k) P_{\sigma v}\left(k_{2}\right)\left(p+p_{1}\right)^{v} \\
& =\left(p+p_{1}\right)^{\mu} P_{\mu \rho_{1}}\left(k_{1}\right) P^{\sigma_{1} \rho_{1}}(k) P_{\sigma_{1} v}\left(k_{1}\right)\left(p+p_{1}\right)^{v} \\
& \approx-\frac{4(\boldsymbol{k}+\boldsymbol{q})^{2}}{x^{2}}\left(k+k_{1}\right)_{2}^{\rho} D_{\rho_{2} \sigma_{2}}^{>}(q)\left(k+k_{2}\right)^{\sigma_{2}} \\
& \approx k^{+} k_{1}^{+} \theta\left(1-\frac{q_{0}^{2}}{\overrightarrow{\mathbf{q}}^{2}}\right) f\left(q_{0}\right) \frac{\boldsymbol{q}^{2}}{\overrightarrow{\mathbf{q}}^{2}} \\
& \quad \times 2 \operatorname{Im}\left[\frac{1}{q^{2}-\Pi_{L}(q)}-\frac{1}{q^{2}-\Pi_{T}(q)}\right]
\end{aligned}
$$

Finally, by using Eqs. (L2), (L5), and (L6), and after performing the same procedure as in the previous appendices,
Eq. (L1) reduces to

$$
\begin{aligned}
M_{1,2, C}= & 8 \operatorname{LTg}^{4}\left[t_{a}, t_{c}\right]\left[t_{c}, t_{a}\right] \int \frac{d^{3} p}{(2 \pi)^{3} 2 E}|J(p)|^{2} \\
& \times \int \frac{d^{3} k}{(2 \pi)^{3} 2 \omega} \frac{d^{2} q}{(2 \pi)^{2}} \frac{\mu^{2}}{q^{2}\left(q^{2}+\mu^{2}\right)} \\
& \times \frac{(\boldsymbol{k}+\boldsymbol{q})^{2}}{\left[(\boldsymbol{k}+\boldsymbol{q})^{2}+\chi\right]^{2}}\left[1-\frac{\sin (\zeta L)}{\zeta L}\right] .
\end{aligned}
$$

\section{APPENDIX M: COMPUTATION OF DIAGRAMS $M_{1,2, R}$ AND $M_{1,2, L}$}

In this appendix, we will calculate the cut diagrams $M_{1,2, R}$ and $M_{1,2, L}$, which are shown in Fig. 12 . We start with $M_{1,2, R}$ :

$$
\begin{aligned}
M_{1,2, R}= & \int \prod_{i=0}^{5} d x_{i} J\left(x_{0}\right) \Delta_{++}^{+}\left(x_{1}-x_{0}\right) v_{\mu}^{+}\left(x_{1}\right) \\
& \times D_{++}^{+\mu \rho_{1}}\left(x_{2}-x_{1}\right) v_{\rho_{1} \rho_{2} \rho_{3}}^{+}\left(x_{2}\right) D_{++}^{+\rho_{3} \sigma_{3}}\left(x_{3}-x_{2}\right)
\end{aligned}
$$



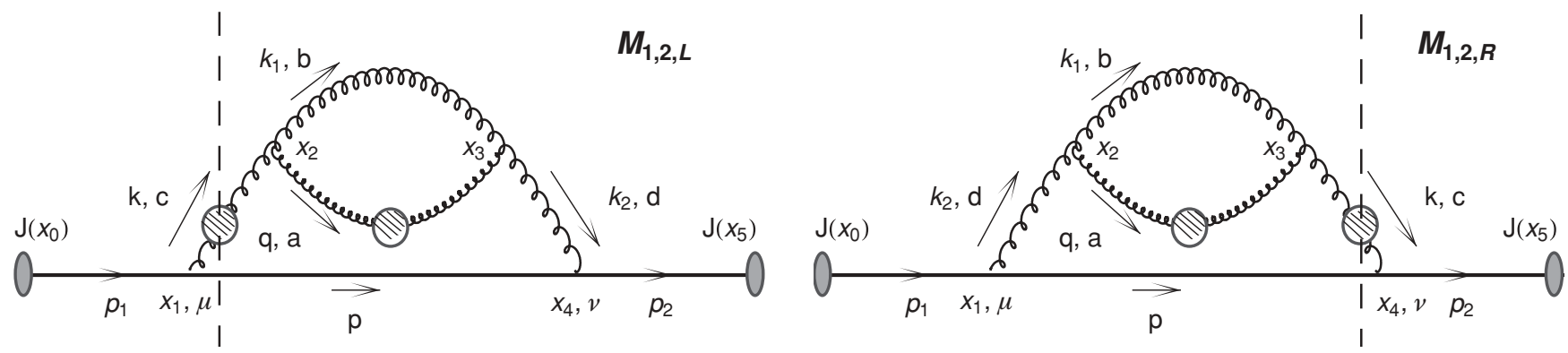

FIG. 12. Feynman diagram $M_{1,2}$ contributing to the radiative energy loss to first order in opacity, labeled the same way as in Fig. 1.

$$
\begin{aligned}
& \times D_{++}^{+\rho_{2} \sigma_{2}}\left(x_{3}-x_{2}\right) \Delta_{-+}\left(x_{4}-x_{1}\right) v_{\sigma_{1} \sigma_{2} \sigma_{3}}^{+}\left(x_{3}\right) \\
& \times D_{-+}^{\sigma_{1} v}\left(x_{4}-x_{3}\right) v_{v}^{-}\left(x_{4}\right) \Delta_{--}^{-}\left(x_{5}-x_{4}\right) J\left(x_{5}\right) \\
& \times \theta\left(x_{1}^{+}-x_{0}^{+}\right) \theta\left(x_{2}^{+}-x_{1}^{+}\right) \theta\left(x_{3}^{+}-x_{2}^{+}\right) \\
& \times \theta\left(x_{4}^{+}-x_{5}^{+}\right) \theta\left[2 L-\left(x_{2}-x_{0}\right)\right] \\
\approx & -\int_{0}^{\infty} \int_{-\infty}^{\infty} \prod_{i=1}^{2} \frac{d p_{i}^{+} d^{2} p_{i}}{(2 \pi)^{3} 2 p_{i}^{+}} \frac{d k_{i}^{+} d^{2} k_{i}}{(2 \pi)^{3} 2 k_{i}^{+}} \\
& \times \int^{3} \frac{d^{3} k}{(2 \pi)^{3} 2 \omega} \frac{d^{3} p}{(2 \pi)^{3} 2 E} \frac{d^{4} q}{(2 \pi)^{4}} g^{4} f^{c b a} t_{c} f^{a b d} t_{d} \\
& \times\left[\left(k_{1}+k_{2}\right)^{\rho} D_{\rho \sigma}^{>}(q)\left(k+k_{1}\right)^{\sigma}\right]\left[\left(p+p_{1}\right)^{\mu}\right. \\
& \left.\times P_{\mu \rho_{1}}\left(k_{2}\right) P^{\sigma_{1} \rho_{1}}\left(k_{1}\right) P_{\rho_{1} v}(k)\left(p+p_{2}\right)^{\nu}\right] I,
\end{aligned}
$$

where in the second step we keep only the dominant parts from triple gluon vertices (see Ref. [8]).

In Eq. (M1) I corresponds to

$$
\begin{aligned}
I= & \int \prod_{i=0}^{5} d x_{i} \theta\left(x_{1}^{+}-x_{0}^{+}\right) \theta\left(x_{2}^{+}-x_{1}^{+}\right) \theta\left(2 L-\left(x_{2}-x_{0}\right)^{+}\right) \\
& \times \theta\left(x_{3}^{+}-x_{2}^{+}\right) \theta\left(x_{4}^{+}-x_{5}^{+}\right) e^{-i p_{1}\left(x_{1}-x_{0}\right)} \\
& \times e^{-i k_{2}\left(x_{2}-x_{1}\right)} e^{-i k_{1}\left(x_{3}-x_{2}\right)} e^{-i q\left(x_{3}-x_{2}\right)} e^{-i p\left(x_{4}-x_{1}\right)} \\
& \times e^{-i k\left(x_{4}-x_{3}\right)} e^{-i p_{2}\left(x_{5}-x_{4}\right)} J\left(x_{0}\right) J\left(x_{5}\right) \\
= & |J(p)|^{2}(2 \pi)^{3} \delta\left[\left(p_{1}-p-k_{2}\right)^{+}\right] \delta^{2}\left(\boldsymbol{p}_{1}-\boldsymbol{p}-\boldsymbol{k}_{2}\right)(2 \pi)^{3} \\
& \times \delta\left[\left(k_{2}-k\right)^{+}\right] \delta^{2}\left(\boldsymbol{k}_{2}-\boldsymbol{k}\right)(2 \pi)^{3} \delta\left[\left(k_{1}+q-k\right)^{+}\right] \\
& \times \delta^{2}\left(\boldsymbol{k}_{1}+\boldsymbol{q}-\boldsymbol{k}\right)(2 \pi)^{3} \delta\left[\left(p_{2}-k-p\right)^{+}\right] \\
& \times \delta^{2}\left(\boldsymbol{p}_{2}-\boldsymbol{p}-\boldsymbol{k}\right) I_{1}
\end{aligned}
$$

and where

$$
\begin{aligned}
I_{1}= & \int_{0}^{2 L} d x_{2}^{\prime+} e^{-\frac{i}{2}\left(k_{2}-k\right)^{-} x_{2}^{\prime+}} \int_{0}^{x_{2}^{\prime+}} d x_{1}^{\prime+} e^{-\frac{i}{2}\left(p_{1}-p-k_{2}\right)^{-} x_{1}^{\prime+}} \\
& \times \int_{0}^{\infty} d x_{3}^{\prime+} e^{-\frac{i}{2}\left(k_{1}+q-k\right)^{-} x_{3}^{\prime+}} \int_{0}^{\infty} d x_{4}^{\prime+} e^{\frac{i}{2}\left(p_{2}-p-k\right)^{-} x_{4}^{\prime+}} .
\end{aligned}
$$

In the last equation, we defined $x_{1}^{\prime+}=x_{1}^{+}-x_{0}^{+}, x_{2}^{\prime+}=x_{2}^{+}-$ $x_{0}^{+}, x_{3}^{\prime+}=x_{3}^{+}-x_{2}^{+}$, and $x_{4}^{\prime+}=x_{4}^{+}-x_{5}^{+}$.

After applying $\delta$ functions from Eq. (M2) and by using Eq. (C5), we obtain $k^{-}=k_{2}^{-}$, which reduces the Eq. (M3) to

$$
I_{1}=\int_{0}^{2 L} d x_{2}^{\prime+} \int_{0}^{x_{2}^{\prime+}} e^{\frac{i}{2} \xi x_{1}^{\prime+}} d x_{1}^{\prime+} \int_{0}^{\infty} d x_{4}^{\prime+} e^{-\frac{i}{2} \xi x_{4}^{\prime+}}
$$

$$
\begin{aligned}
& \times \int_{0}^{\infty} d x_{3}^{\prime+} e^{-i q^{-} y} \\
= & \frac{16 L}{\xi^{2}}\left[1-\frac{\sin \xi L}{\xi L}+i \frac{1-\cos \xi L}{\xi L}\right] \int_{0}^{\infty} d y e^{-i q^{-} y} .
\end{aligned}
$$

In the above equation, we also used $\left(p_{1}-k-p\right)^{-}=\left(p_{2}-\right.$ $p-k)^{-}=-\xi,\left(k_{1}+q-k\right)^{-} \approx q^{-}$and $y \equiv \frac{x_{3}^{\prime+}}{2}$.

Similarly as in Ref. [8], for highly energetic jets

$$
\begin{aligned}
& \left(p+p_{1}\right)^{\mu} P_{\mu \rho_{1}}\left(k_{2}\right) P^{\rho_{1} \sigma_{1}}(k) P_{\sigma_{1} v}(k)\left(p+p_{2}\right)^{v}=-\frac{4 \boldsymbol{k}^{2}}{\xi^{2}} \\
& \left(k+k_{1}\right)^{\rho} D_{\rho \sigma}^{>}(q)\left(k+k_{1}\right)^{\sigma} \approx k^{+} k_{1}^{+} \theta\left(1-\frac{q_{0}^{2}}{\overrightarrow{\mathbf{q}}^{2}}\right) f\left(q_{0}\right) \frac{\boldsymbol{q}^{2}}{\overrightarrow{\mathbf{q}}^{2}} \\
& \quad \times 2 \operatorname{Im}\left[\frac{1}{q^{2}-\Pi_{L}(q)}-\frac{1}{q^{2}-\Pi_{T}(q)}\right] .
\end{aligned}
$$

Finally, by using Eqs. (M2), (M4), and (M5), and after performing the same procedure as in the previous appendices, Eq. (M1) reduces to

$$
\begin{aligned}
M_{1,2, R}= & -4 L T g^{4}\left[t_{c}, t_{a}\right]\left[t_{a}, t_{c}\right] \int \frac{d^{3} p}{(2 \pi)^{3} 2 E}|J(p)|^{2} \\
& \times \int \frac{d^{3} k}{(2 \pi)^{3} 2 \omega} \frac{d^{4} q}{(2 \pi)^{4}} \frac{\boldsymbol{k}^{2}}{\left(\boldsymbol{k}^{2}+\chi\right)^{2}}\left(1-\frac{\sin \xi L}{\xi L}\right. \\
& \left.+i \frac{1-\cos \xi L}{\xi L}\right) \int_{0}^{\infty} d y e^{-i q^{-} y} \theta\left(1-\frac{q_{0}^{2}}{\overrightarrow{\mathbf{q}}^{2}}\right) \frac{1}{q_{0}} \\
& \times \frac{\boldsymbol{q}^{2}}{\overrightarrow{\mathbf{q}}^{2}} 2 \operatorname{Im}\left[\frac{1}{q^{2}-\Pi_{L}(q)}-\frac{1}{q^{2}-\Pi_{T}(q)}\right] .
\end{aligned}
$$

Finally, by using that $M_{1,2, L}$ is a complex conjugate of $M_{1,2, R}$, and by performing the same procedure as in Eqs. (D24)-(D28), one obtains

$$
\begin{aligned}
M_{1,2, R}+M_{1,2, L}= & -4 L T g^{4}\left[t_{a}, t_{c}\right]\left[t_{c}, t_{a}\right] \int \frac{d^{3} p}{(2 \pi)^{32 E}}|J(p)|^{2} \\
& \times \int \frac{d^{3} k}{(2 \pi)^{3} 2 \omega} \frac{d^{2} q}{(2 \pi)^{2}} \frac{\mu^{2}}{\boldsymbol{q}^{2}\left(\boldsymbol{q}^{2}+\mu^{2}\right)} \\
& \times \frac{\boldsymbol{k}^{2}}{\left(\boldsymbol{k}^{2}+\chi\right)^{2}}\left(1-\frac{\sin \xi L}{\xi L}\right) .
\end{aligned}
$$




\section{APPENDIX N: COMPUTATION OF THE TADPOLE DIAGRAMS}

In this appendix we calculate tadpole diagrams shown in Fig. 13 and show that they present negligible contribution to the radiative energy loss.

$$
\begin{aligned}
M_{t, R}= & \int \prod_{i=0}^{4} d x_{i} J\left(x_{0}\right) \Delta_{++}^{+}\left(x_{1}-x_{0}\right) v_{\mu}^{+}\left(x_{1}\right) D_{++}^{+\mu \rho}\left(x_{2}-x_{1}\right) \\
& \times v_{\rho \sigma \lambda \tau}^{+}\left(x_{2}\right) D_{++}^{+\lambda \tau}\left(x_{2}\right) D_{-+}^{\sigma \nu}\left(x_{3}-x_{2}\right) v_{v}^{-}\left(x_{3}\right) \\
& \times \Delta_{-+}\left(x_{3}-x_{1}\right) \Delta_{--}^{-}\left(x_{4}-x_{3}\right) J\left(x_{4}\right) \theta\left(x_{1}^{+}-x_{0}^{+}\right) \\
& \times \theta\left(x_{2}^{+}-x_{1}^{+}\right) \theta\left[2 L-\left(x_{2}-x_{0}\right)^{+}\right] \theta\left(x_{3}^{+}-x_{4}^{+}\right),
\end{aligned}
$$

where $v_{\rho \sigma \lambda \tau}^{+}\left(x_{2}\right)=-g^{2}\left[f_{c e a} f_{c e b}\left(2 g_{\rho \sigma} g_{\lambda \tau}-g_{\rho \lambda} g_{\sigma \tau}-g_{\rho \tau} g_{\sigma \lambda}\right)\right]$, leading to

$$
\begin{aligned}
M_{t, R}= & \prod_{i=0}^{4} d x_{i} J\left(x_{0}\right) \int_{-\infty}^{\infty} \int_{0}^{\infty} \frac{d p_{1}^{+} d^{2} p_{1}}{(2 \pi)^{3} 2 p_{1}^{+}} e^{-i p_{1}\left(x_{1}-x_{0}\right)} \\
& \times \int \frac{d^{3} p}{(2 \pi)^{3} 2 E} e^{-i p\left(x_{3}-x_{1}\right)}\left[-i g\left(p+p_{1}\right)_{\mu} t_{a}\right](-1) \\
& \times \int_{-\infty}^{\infty} \int_{0}^{\infty} \frac{d k_{1}^{+} d^{2} k_{1}}{(2 \pi)^{3} 2 k_{1}^{+}} e^{-i k_{1}\left(x_{2}-x_{1}\right)} P^{\mu \rho}\left(k_{1}\right)\left(-g^{2}\right) \\
& \times f^{c e a} f_{c e b}\left(2 g_{\rho \sigma} g_{\lambda \tau}-g_{\rho \lambda} g_{\sigma \tau}-g_{\rho \tau} g_{\sigma \lambda}\right) \\
& \times \int \frac{d^{4} q}{(2 \pi)^{4}} D^{\lambda \tau>}(q)(-1) \int \frac{d^{3} k}{(2 \pi)^{3} 2 \omega} e^{-i k\left(x_{3}-x_{2}\right)} \\
& \times P^{\sigma \nu}(k) \int \frac{d p_{2}^{+} d^{2} p_{2}}{(2 \pi)^{3} 2 p_{2}^{+}} e^{-i p_{2}\left(x_{4}-x_{3}\right)}\left(i g\left(p+p_{2}\right)_{v} t_{b}\right) \\
& \times \theta\left(x_{1}^{+}-x_{0}^{+}\right) \theta\left(x_{2}^{+}-x_{1}^{+}\right) \theta\left[2 L-\left(x_{2}-x_{0}\right)^{+}\right] \\
& \times \theta\left(x_{3}^{+}-x_{4}^{+}\right) J\left(x_{4}\right) \\
= & \int \frac{d^{3} k}{(2 \pi)^{3} 2 \omega} \frac{d^{3} p}{(2 \pi)^{3} 2 E} \frac{d^{4} q}{(2 \pi)^{4}} \prod_{i=1}^{2} \frac{d p_{i}^{+} d^{2} p_{i}}{(2 \pi)^{3} 2 p_{i}^{+}} \\
& \times \frac{d k_{i}^{+} d^{2} k_{i}}{(2 \pi)^{3} 2 k_{i}^{+}} g^{4}\left(-f^{c e a} t_{a} f^{c e b} t_{b}\right)\left(p+p_{1}\right)_{\mu} P^{\mu \rho}\left(k_{1}\right) \\
& \times\left(2 g_{\rho \sigma} g_{\lambda \tau}-g_{\rho \lambda} g_{\sigma \tau}-g_{\rho \tau} g_{\sigma \lambda}\right) \\
& \times D^{\tau \lambda>}(q) P^{\sigma v}(k)\left(p+p_{2}\right)_{v} I,
\end{aligned}
$$

where

$$
\begin{aligned}
I= & \int \prod_{i=0}^{4} d x_{i} J\left(x_{0}\right) J\left(x_{4}\right) e^{-i p_{1}\left(x_{1}-x_{0}\right)} e^{-i k_{1}\left(x_{2}-x_{1}\right)} e^{-i k\left(x_{3}-x_{2}\right)} \\
& \times e^{-i p\left(x_{3}-x_{1}\right)} e^{-i p_{2}\left(x_{4}-x_{3}\right)} \theta\left(x_{1}^{+}-x_{0}^{+}\right) \\
& \times \theta\left(x_{2}^{+}-x_{1}^{+}\right) \theta\left(2 L-\left(x_{2}-x_{1}\right)^{+}\right) \theta\left(x_{3}^{+}-x_{4}^{+}\right) \\
= & |J(p)|^{2}(2 \pi)^{3} \delta\left[\left(p_{1}-p-k_{1}\right)^{+}\right] \delta^{2}\left(\boldsymbol{p}_{1}-\boldsymbol{p}-\boldsymbol{k}_{1}\right) \\
& \times(2 \pi)^{3} \delta\left[\left(k_{1}-k\right)^{+}\right] \delta^{2}\left(\boldsymbol{k}_{1}-\boldsymbol{k}\right) \\
& \times(2 \pi)^{3} \delta\left[\left(p_{2}-p-k\right)^{+}\right] \delta^{2}\left(\boldsymbol{p}_{2}-\boldsymbol{p}-\boldsymbol{k}\right) I_{1}
\end{aligned}
$$

and where

$$
\begin{aligned}
I_{1}= & \int_{0}^{2 L} d x_{2}^{\prime+} e^{-\frac{i}{2}\left(k_{1}-k\right)^{-} x_{2}^{\prime+}} \int_{0}^{x_{2}^{\prime+}} d x_{1}^{\prime+} e^{-\frac{i}{2}\left(p_{1}-p-k_{1}\right)^{-} x_{1}^{\prime+}} \\
& \times \int_{0}^{\infty} d x_{3}^{\prime+} e^{+\frac{i}{2}\left(p_{2}-p-k\right)^{-} x_{3}^{\prime+}} .
\end{aligned}
$$

Here $x_{1}^{\prime+}=x_{1}^{+}-x_{0}^{+}, x_{2}^{\prime+}=x_{2}^{+}-x_{0}^{+}$, and $x_{3}^{\prime+}=x_{3}^{+}-x_{4}^{+}$. Also, by using $\delta$ functions from Eq. (N3) and Eq. (C5) it follows that

$$
\begin{aligned}
p_{2} & =p_{1} ; \quad k=k_{1} ; \\
\left(p_{1}-p-k\right)^{-} & =\left(p_{2}-p-k\right)^{-} \approx-\xi .
\end{aligned}
$$

After using relations from (N5), Eq. (N4) becomes

$$
\begin{aligned}
I_{1} & =\int_{0}^{2 L} d x_{2}^{\prime+} \int_{0}^{x_{2}^{\prime+}} e^{\frac{i}{2} \xi x_{1}^{\prime+}} \int_{0}^{\infty} d x_{3}^{\prime+} e^{-\frac{i}{2} \xi x_{3}^{\prime+}} \\
& =\frac{8 L}{\xi^{2}}\left[1-\frac{\sin \xi L}{\xi L}+i \frac{1-\cos \xi L}{\xi L}\right] .
\end{aligned}
$$

By using Eqs. (N6) and (N3), Eq. (N2) becomes

$$
\begin{aligned}
M_{t R}= & 8 g^{4} L\left[t_{a}, t_{c}\right]\left[t_{c}, t_{a}\right] \int \frac{d^{3} p}{(2 \pi)^{3} 2 E}|J(p)|^{2} \\
& \times \int \frac{d^{3} k}{(2 \pi)^{3} 2 \omega} \frac{d^{4} q}{(2 \pi)^{4}} \frac{1}{\left(2 E^{+}\right)^{2}} \frac{1}{2 k_{1}^{+}} \\
& \times\left[8 p^{\mu} P_{\mu \rho}(k) P_{\nu}^{\rho}(k) p^{\nu} D_{\lambda}^{>\lambda}(q)-8 p^{\mu} P_{\mu \rho}(k) D^{\rho \sigma>}(q)\right. \\
& \left.\times P_{\sigma \nu}(k) p^{\nu}\right] \frac{x^{2} E^{+2}}{\left(k^{2}+\chi\right)^{2}}\left[1-\frac{\sin \xi L}{\xi L}+i \frac{1-\cos \xi L}{\xi L}\right] .
\end{aligned}
$$
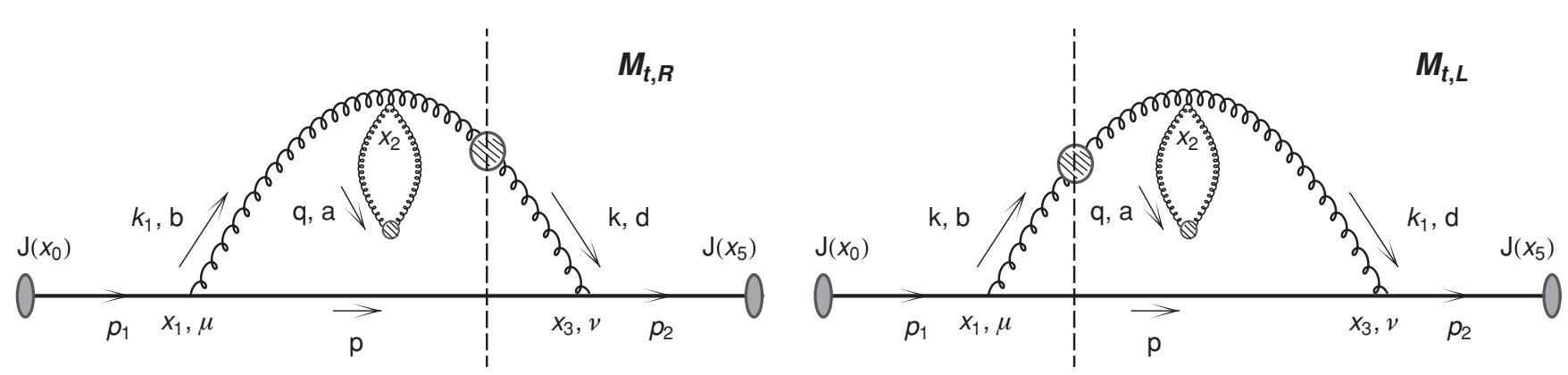

FIG. 13. Tadpole Feynman diagrams $M_{t, R}$ and $M_{t, L}$, labeled the same way as in Fig. 1. 
To proceed further, let us first calculate

$$
\begin{aligned}
& p^{\mu} P_{\mu \rho}(k) P_{\nu}^{\rho}(k) p^{v} D_{\lambda}^{>\lambda}(q)-p^{\mu} P_{\mu \rho}(k) D^{\rho \sigma>}(q) P_{\sigma \nu}(k) p^{v} \\
& \quad=p^{\mu} P_{\mu \nu}(k) p^{v} D_{\lambda}^{>\lambda}(q)-p^{\mu} P_{\mu \rho}(k) D^{\rho \sigma>}(q) P_{\sigma \nu}(k) p^{v} .
\end{aligned}
$$

We here use

$$
\begin{aligned}
p^{\mu} P_{\mu \rho}(k) P_{\nu}^{\rho}(k) p^{v} & =-\frac{\boldsymbol{k}^{2}}{x^{2}} ; \quad P_{\lambda}^{\lambda}(q)=2 ; \\
Q_{\lambda}^{\lambda}(q) & =1-\frac{q_{0}^{2}}{q^{2}},
\end{aligned}
$$

leading to

$$
\begin{aligned}
D_{\lambda}^{>\lambda}(q)= & \theta\left(1-\frac{q_{0}^{2}}{\overrightarrow{\mathbf{q}}^{2}}\right)\left[1+f\left(q^{0}\right)\right] \\
& \times 2 \operatorname{Im}\left[\frac{2}{q^{2}-\Pi_{T}(q)}+\frac{1-\frac{q_{0}^{2}}{\overline{\mathbf{q}}_{2}}}{q^{2}-\Pi_{L}(q)}\right] .
\end{aligned}
$$

Furthermore, in Coulomb gauge

$$
P_{\mu \rho}(k) Q^{\rho \sigma}(q) P_{\sigma \lambda}(k) \equiv 0,
$$

leading to

$$
\begin{aligned}
p^{\mu} & P_{\mu \rho}(k) D^{\rho \sigma>}(q) P_{\sigma \nu}(k) p^{\nu} \\
= & p^{\mu} P_{\mu \rho}(k) P^{\rho \sigma}(q) P_{\sigma \lambda} p^{\nu} \\
= & -p^{2}+\frac{(p k)^{2}}{k^{2}}+\frac{(p q)^{2}}{q^{2}}-2 \frac{(p k)(p q)(q k)}{k^{2} q^{2}}+\frac{(p k)(q k)^{2}}{\left(k^{2}\right)^{2} q^{2}} \\
= & -\frac{\boldsymbol{k}^{2}}{x^{2}} \theta\left(1-\frac{q_{0}^{2}}{\overrightarrow{\mathbf{q}}^{2}}\right)\left[1+f\left(q^{0}\right)\right] \\
\quad & 2 \operatorname{Im}\left[\frac{1+\frac{(q \dot{k})^{2}}{\boldsymbol{k}^{2} \overrightarrow{\mathbf{q}}^{2}}}{q^{2}-\Pi_{T}(q)}+\frac{1-\frac{q_{0}^{2}}{\overline{\mathbf{q}}_{2}}}{q^{2}-\Pi_{L}(q)}\right] .
\end{aligned}
$$

By using Eqs. (N8)-(N12), Eq. (N7) becomes

$$
\begin{aligned}
M_{t, R}= & -8 L g^{4}\left[t_{a}, t_{c}\right]\left[t_{c}, t_{a}\right] \int \frac{d^{3} p}{(2 \pi)^{3} 2 E}|J(p)|^{2} \\
& \times \int \frac{d^{3} k}{(2 \pi)^{3} 2 \omega} \frac{d^{4} q}{(2 \pi)^{4}} \frac{\boldsymbol{k}^{2}}{\left(\boldsymbol{k}^{2}+\chi\right)^{2}} \\
& \times\left[1-\frac{\sin \xi L}{\xi L}+i \frac{1-\cos \xi L}{\xi L}\right] \frac{1}{k^{+}} \int \frac{d^{4} q}{(2 \pi)^{4}} \theta \\
& \times\left(1-\frac{q_{0}^{2}}{\overrightarrow{\mathbf{q}}^{2}}\right) \frac{T}{q_{0}} 2 \operatorname{Im}\left[\frac{1-\frac{(\boldsymbol{q} \boldsymbol{k})^{2}}{\boldsymbol{k}^{2} \overrightarrow{\mathbf{q}}^{2}}}{q^{2}-\Pi_{T}(q)}\right] .
\end{aligned}
$$

Because $M_{t, L}$ is a complex conjugate of $M_{t, R}$, one finally obtains

$$
\begin{aligned}
M_{t, R}+M_{t, L}= & 8 L T g^{4}\left[t_{a}, t_{c}\right]\left[t_{c}, t_{a}\right] \\
& \times \int \frac{d^{3} p}{(2 \pi)^{3} 2 E}|J(p)|^{2} \int \frac{d^{3} k}{(2 \pi)^{3} 2 \omega} \\
& \times \frac{\boldsymbol{k}^{2}}{\left(\boldsymbol{k}^{2}+M x^{2}+m g^{2}\right)^{2}}\left[1-\frac{\sin \xi L}{\xi L}\right], I_{t}
\end{aligned}
$$

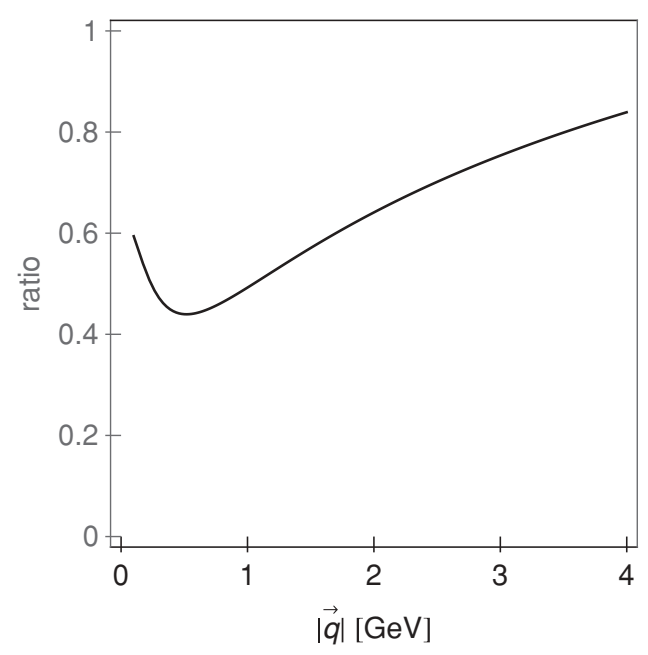

FIG. 14. Ratio $\frac{J_{t, \max }\left(\overrightarrow{\mathbf{q}}^{2}\right)}{v\left(\overrightarrow{\mathbf{q}}^{2}\right)}$ is shown as a function of momentum $|\overrightarrow{\mathbf{q}}|$ for Debye mass $\mu=0.5$.

where

$$
I_{t}=-\frac{2}{\omega} \int \frac{d^{4} q}{(2 \pi)^{4}} \theta\left(1-\frac{q_{0}^{2}}{\overrightarrow{\mathbf{q}}^{2}}\right) \frac{1}{q_{0}} \operatorname{Im}\left[\frac{1-\frac{(q \dot{k})^{2}}{\boldsymbol{k}^{2} \overrightarrow{\mathbf{q}}^{2}}}{q^{2}-\Pi_{T}(q)}\right]
$$

Finally, we want to show that $I_{t} \ll \int \frac{d \boldsymbol{q}^{2}}{4 \pi} v\left(\boldsymbol{q}^{2}\right)$ [where $v\left(\boldsymbol{q}^{2}\right)=$ $\frac{\mu^{2}}{q^{2}\left(q^{2}+\mu^{2}\right)}$, leading to the conclusion that tadpole contribution is negligible to the first order in opacity radiative energy loss. To do this, we first observe that $0 \leqslant \frac{(\boldsymbol{q} \dot{k})^{2}}{k^{2} \overrightarrow{\mathbf{q}}^{2}} \leqslant 1$, leading to

$$
0 \leqslant I_{t} \leqslant I_{t, \max }
$$

where

$$
\begin{aligned}
I_{t, \text { max }} & =-\frac{2}{\omega} \int \frac{d^{4} q}{(2 \pi)^{4}} \theta\left(1-\frac{q_{0}^{2}}{\overrightarrow{\mathbf{q}}^{2}}\right) \frac{1}{q_{0}} 2 \operatorname{Im}\left[\frac{2}{q^{2}-\Pi_{T}(q)}\right] \\
& =\int \frac{d \overrightarrow{\mathbf{q}}^{2}}{4 \pi} \frac{|\overrightarrow{\mathbf{q}}|}{\omega} J_{t, \text { max }},\left(\overrightarrow{\mathbf{q}}^{2}\right),
\end{aligned}
$$

where we defined $y \equiv \frac{q_{0}}{|\overrightarrow{\mathbf{q}}|}$, and

$$
J_{t, \max }\left(\overrightarrow{\mathbf{q}}^{2}\right)=\frac{4}{\pi^{2}} \int_{0}^{1} \frac{d x}{x} \operatorname{Im}\left[\frac{1}{\overrightarrow{\mathbf{q}}^{2}\left(1-x^{2}\right)+\Pi_{T}(x)}\right],
$$

Furthermore, we want to show that $I_{t} \sim \int \frac{d \overrightarrow{\mathbf{q}}^{2}}{4 \pi} \frac{|\overrightarrow{\mathbf{q}}|}{\omega} v\left(\overrightarrow{\mathbf{q}}^{2}\right)$. To do this, we have to prove that $J_{t, \max }\left(\overrightarrow{\mathbf{q}}^{2}\right)$ is comparable with $v\left(\overrightarrow{\mathbf{q}}^{2}\right)$. We numerically confirmed that $\frac{J_{t, \max }\left(\overrightarrow{\mathbf{q}}^{2}\right)}{v\left(\overrightarrow{\mathbf{q}}^{2}\right)} \lesssim 1$, as demonstrated in Fig. 14 for typical value of Debye mass $\mu=0.5$. That is, in Fig. 14 we see that the absolute values of the ratios are notably smaller than 1 . We also checked that the same conclusion is valid independently on the value of Debye mass. Having in mind that $\frac{|\overrightarrow{\mathbf{q}}|}{\omega} \ll 1$, and by using $I_{t} \sim \int \frac{d \overrightarrow{\mathbf{q}}^{2}}{4 \pi} \frac{|\overrightarrow{\mathbf{q}}|}{\omega} v\left(\overrightarrow{\mathbf{q}}^{2}\right)$, it becomes evident that $I_{t} \ll \int \frac{d \overrightarrow{\mathbf{q}}^{2}}{4 \pi} v\left(\overrightarrow{\mathbf{q}}^{2}\right)$, which leads to the conclusion that tadpoles present a negligible contribution to the first order in opacity radiative energy loss. 
[1] M. Gyulassy, Lect. Notes Phys. 583, 37 (2002).

[2] M. Gyulassy and M. Plümer, Nucl. Phys. A527, 641 (1991).

[3] M. Gyulassy, M. Plümer, M. Thoma, and X. N. Wang, Nucl. Phys. A538, 37C (1992); X. N. Wang and M. Gyulassy, Phys. Rev. Lett. 68, 1480 (1992).

[4] M. Guylassy, I. Vitev, X. N. Wang, and B. W. Zhang, in Quark Gluon Plasma 3, edited by R. C. Hwa and X. N. Wang (World Scientific, Singapore, 2003), p. 123.

[5] R. Baier, Yu. L. Dokshitzer, A. J. Mueller, and D. Schiff, Phys. Rev. C 58, 1706 (1998); R. Baier, Yu. L. Dokshitzer, A. J. Mueller, S. Peigne, and D. Schiff, Nucl. Phys. B483, 291 (1997).

[6] R. Baier, D. Schiff, and B. G. Zakharov, Annu. Rev. Nucl. Part. Sci. 50, 37 (2000).

[7] A. Kovner and U. A. Wiedemann, in Quark Gluon Plasma 3, edited by R. C. Hwa and X. N. Wang (World Scientific, Singapore, 2003), p. 192.

[8] M. Djordjevic and U. Heinz, Phys. Rev. C 77, 024905 (2008).

[9] M. Djordjevic and U. Heinz, Phys. Rev. Lett. 101, 022302 (2008).

[10] B. G. Zakharov, JETP Lett. 76, 201 (2002).

[11] J. F. Gunion and G. Bertsch, Phys. Rev. D 25, 746 (1982).

[12] A. B. Migdal, Phys. Rev. 103, 1811 (1956); L. D. Landau and I. Pomeranchuk, Dokl. Akad. Nauk Ser. Fiz. 92, 535 (1953); 92, 735 (1953).

[13] M. Djordjevic, Phys. Rev. C 74, 064907 (2006).

[14] M. Djordjevic and M. Gyulassy, Phys. Rev. C 68, 034914 (2003).

[15] J. I. Kapusta, Finite-Temperature Field Theory (Cambridge University Press, New York, 1989).

[16] M. Le Bellac, Thermal Field Theory (Cambridge University Press, New York, 1996).

[17] M. Gyulassy, P. Levai, and I. Vitev, Nucl. Phys. B594, 371 (2001).

[18] M. Djordjevic and M. Gyulassy, Phys. Lett. B560, 37 (2003); Nucl. Phys. A733, 265 (2004).

[19] E. Braaten and M. H. Thoma, Phys. Rev. D 44, R2625 (1991).

[20] E. Braaten and M. H. Thoma, Phys. Rev. D 44, 1298 (1991).
[21] M. H. Thoma and M. Gyulassy, Nucl. Phys. B351, 491 (1991).

[22] O. K. Kalashnikov and V. V. Klimov, Sov. J. Nucl. Phys. 31, 699 (1980).

[23] V. V. Klimov, Sov. Phys. JETP 55, 199 (1982).

[24] H. A. Weldon, Phys. Rev. D 26, 1394 (1982).

[25] U. Heinz, Ann. Phys. (NY) 168, 148 (1986).

[26] R. D. Pisarski, Physica A 158, 146 (1989).

[27] A. Rebhan, Lect. Notes Phys. 583, 161 (2002).

[28] A. V. Selikhov and M. Gyulassy, Phys. Lett. B316, 373 (1993); Phys. Rev. C 49, 1726 (1994).

[29] M. Gyulassy and X. N. Wang, Nucl. Phys. B420, 583 (1994); X. N. Wang, M. Gyulassy, and M. Plumer, Phys. Rev. D 51, 3436 (1995).

[30] U. A. Wiedemann, Nucl. Phys. B588, 303 (2000); B582, 409 (2000).

[31] E. Wang and X. N. Wang, Phys. Rev. Lett. 87, 142301 (2001); X. N. Wang and X. F. Guo, Nucl. Phys. A696, 788 (2001); X. F. Guo and X. N. Wang, Phys. Rev. Lett. 85, 3591 (2000).

[32] N. Armesto, C. A. Salgado, and U. A. Wiedemann, Phys. Rev. D 69, 114003 (2004).

[33] M. Djordjevic, Phys. Rev. C 73, 044912 (2006).

[34] P. Arnold, G. D. Moore, and L. G. Yaffe, J. High Energy Phys. 11 (2001) 057; 06 (2002) 030; 01 (2003) 030.

[35] X. N. Wang, Phys. Lett. B485, 157 (2000).

[36] Note that in the widely used static approximation this problem is absent: only longitudinally polarized gluons contribute to the static potential (see e.g. Refs. [16,17,29]), leading to individually convergent results for each of the scattering diagrams.

[37] S. Wicks, W. Horowitz, M. Djordjevic, and M. Gyulassy, Nucl. Phys. A784, 426 (2007).

[38] P. Aurenche, F. Gelis, and H. Zaraket, J. High Energy Phys. 05 (2002) 043.

[39] A. Adil, M. Gyulassy, W. A. Horowitz, and S. Wicks, Phys. Rev. C 75, 044906 (2007)

[40] J. B. Kogut and D. E. Soper, Phys. Rev. D 1, 2901 (1970).

[41] A. Das, Finite Temperature Field Theory (World Scientific, Singapore, 1997). 